University of Louisville

ThinkIR: The University of Louisville's Institutional Repository

Electronic Theses and Dissertations

$5-2007$

\title{
Microtacks for retinal implant applications.
}

Scott Douglas Cambron 1980-

University of Louisville

Follow this and additional works at: https://ir.library.louisville.edu/etd

\section{Recommended Citation}

Cambron, Scott Douglas 1980-, "Microtacks for retinal implant applications." (2007). Electronic Theses and Dissertations. Paper 201.

https://doi.org/10.18297/etd/201

This Master's Thesis is brought to you for free and open access by ThinkIR: The University of Louisville's Institutional Repository. It has been accepted for inclusion in Electronic Theses and Dissertations by an authorized administrator of ThinkIR: The University of Louisville's Institutional Repository. This title appears here courtesy of the author, who has retained all other copyrights. For more information, please contact thinkir@louisville.edu. 


\author{
By \\ Scott Douglas Cambron \\ B.S., University of Louisville, 2003 \\ A Thesis \\ Submitted to the Faculty of the \\ University of Louisville \\ As Partial Fulfillment of the Requirements \\ For the Professional Degree \\ MASTER OF ENGINEERING
}

Department of Mechanical Engineering

May 2007 


\title{
MICROTACKS FOR RETINAL IMPLANT APPLICATIONS
}

Submitted by:

\author{
Scott Douglas Cambron
}

A Thesis Approved on

April 23, 2007

by the Following Reading and Examination Committee

Robert S. Keynton, Thesis Director

William Hnat

Kevin M. Walsh 


\section{ACKNOWLEDGEMENTS}

I would like to first thank my parents, Doug and Janie Cambron, and my sisters, Terri Lynn, Renee, Ramona, Regina and Kimberly for being there through thick and thin, and understanding the sacrifices that needed to be made in order for me to succeed with my education, career and life. Their support has meant the world to me.

Thanks to Dr. Jose Franco-Sarabia for fabricating all of the silicon microtacks for my testing, to Dr. Volker Enzmann for allowing me to sit in on a couple of surgeries to see firsthand how a retinal prosthesis was implanted and tested, to Swift \& Company for the continuous donation of porcine eyes, and to Dr. Roger Bradshaw and Kaushik Bindiganavile-Nagendran for allowing me to use the Rheometric Series RSA III. Special thanks to Tommy Roussel and Alex Isham for all of their input throughout this project and putting up with all of my disruptive questions. Thanks to Dr. William Hnat for his assistance with fabricating and calibrating my initial ring cell designs and also being a part of my defense committee. Thanks to Dr. Kevin Walsh for all of his input on the microfabrication processes and also being a part of my defense committee.

Most of all I am greatly appreciative to Dr. Robert Keynton for not only being my advisor throughout this arduous and ongoing thesis project but also giving that 19 year old "kid" a chance by giving me the opportunity as an undergraduate research assistant so long ago, to make all this happen. He has undoubtedly assisted in molding me into not only the researcher/engineer that I am today, but also the person as well. Thanks again for your guidance, patience and friendship... Dr. K. 


\section{TABLE OF CONTENTS}

ABSTRACT vi

LIST OF TABLES vii

LIST OF FIGURES viii

1. INTRODUCTION 1

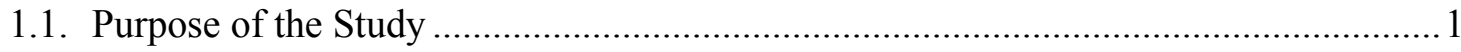

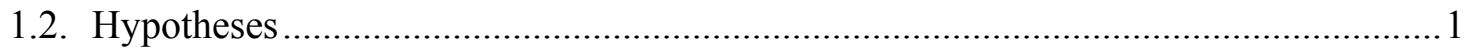

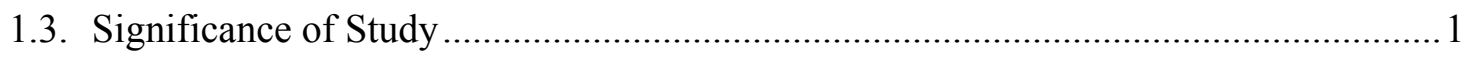

2. LITERATURE REVIEW 2

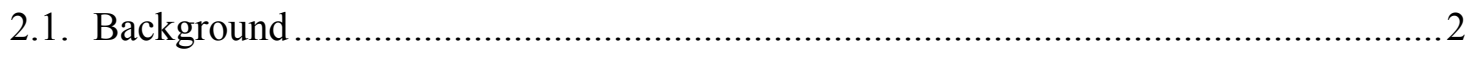

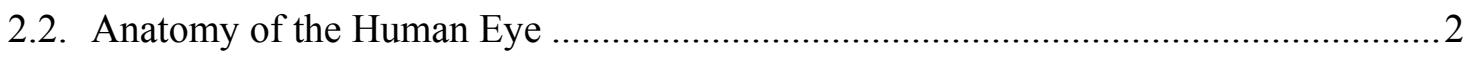

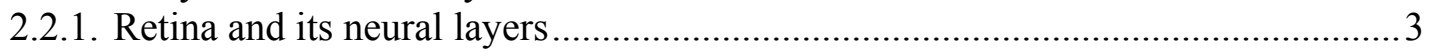

2.2.1.1. Photoreceptors 3

2.2.1.2. Bipolar Cells 4

2.2.1.3. Ganglion Cells 5

2.2.1.4. Macula 6

2.2.1.5. Retinal Pigment Epithelium (RPE) and Bruch's Membrane (BM) 6

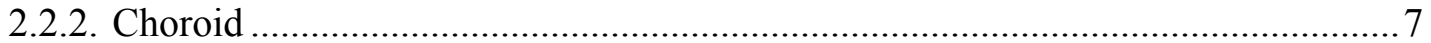

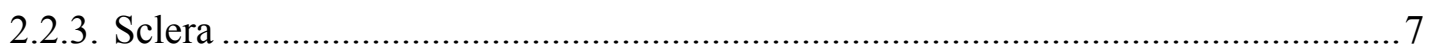

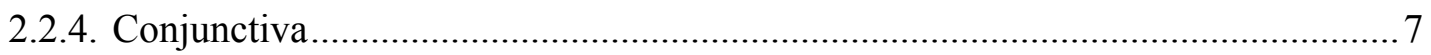

2.3. Age Related Macular Degeneration .......................................................................... 8

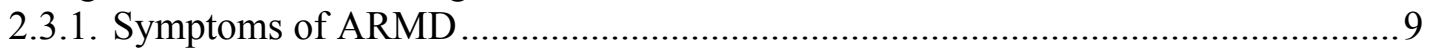

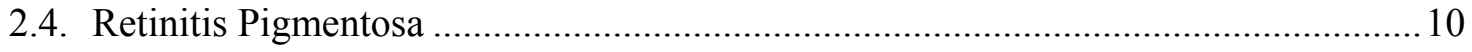

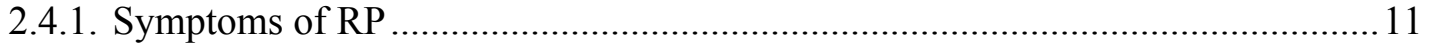

2.5. Treatment Possibilities for ARMD and RP........................................................... 11

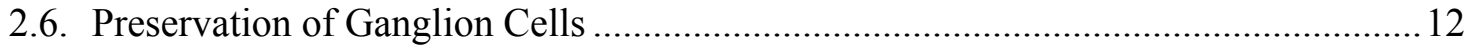

2.7. Current Visual Prosthetic Possibilities......................................................................... 13

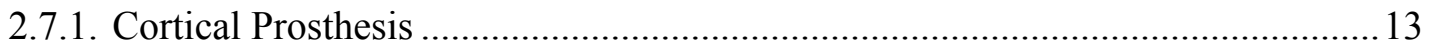

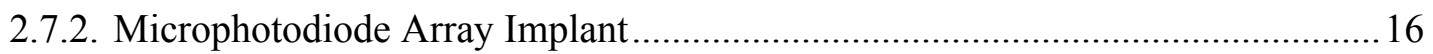

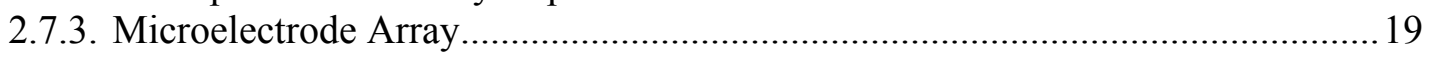

2.7.3.1. Fastening of MEA to Retinal Surface $\quad 21$

3. INSTRUMENTATION AND EQUIPMENT 24

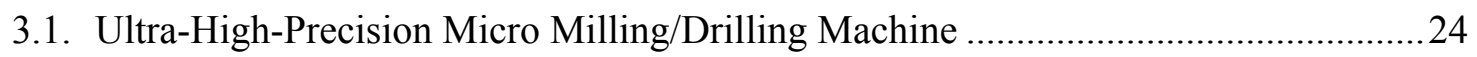

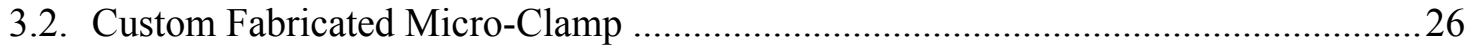

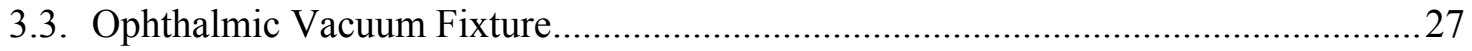

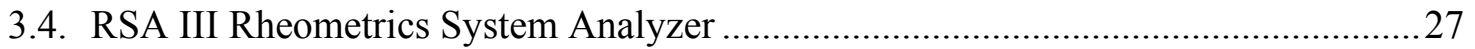


3.5. WYKO Metrology System

3.6. Zeiss SUPRATM 35VP Scanning Electron Microscope ........................................30

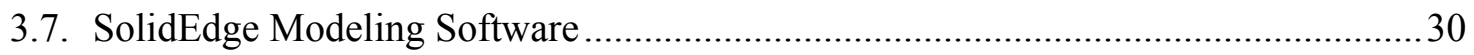

4. PROCEDURES 31

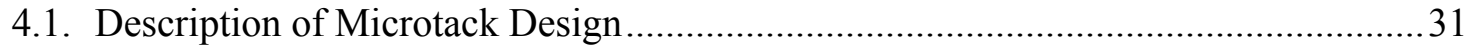

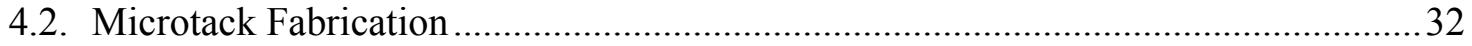

4.2.1. Silicon Microtack using DRIE and Microfabrication Techniques ....................... 32

4.2.2. Sharp Tip 3-D Microtack Fabrication Technique .............................................. 37

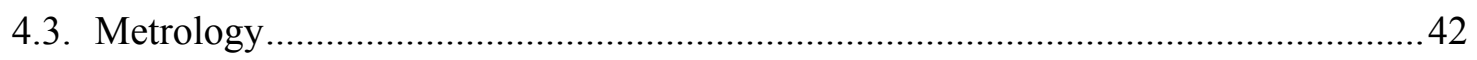

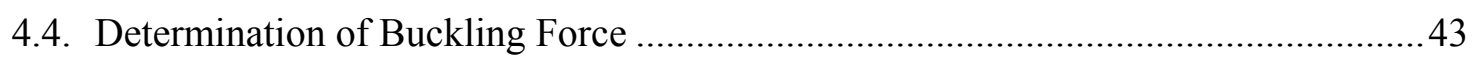

4.5. Characterization of Microtack Performance in Fiber Reinforced Silicone Rubber

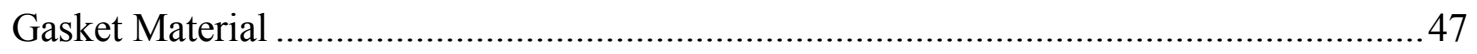

4.6. Characterization of the Microtack Performance in a Porcine Eye............................49

4.7. Insertion and Retention Force Experiments...................................................50

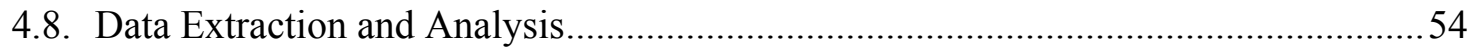

5. RESULTS AND DISCUSSION 57

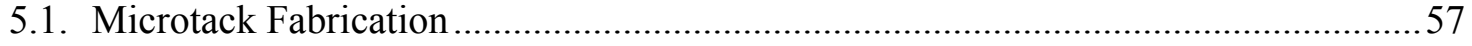

5.1.1. Fabrication of Chisel Tip and Pointed Tip Silicon Microtacks ...........................57

5.1.2. Fabrication of 3-D Sharp Tip Titanium Microtacks ...........................................59

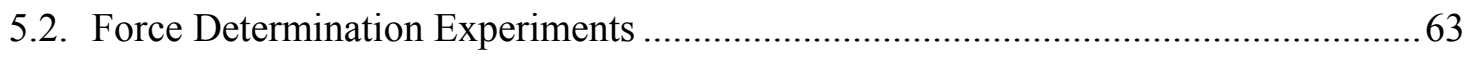

5.2.1. Characterization of Microtack Performance in a Fiber Reinforced Silicone

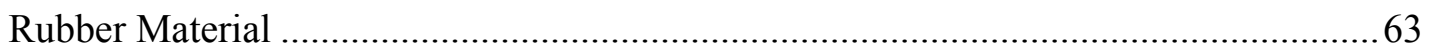

5.2.2. Characterization of the Microtack Performance in a Porcine Eye........................ 71

5.2.3. Discussion of Variance Between Samples...................................................... 75

6. CONCLUSIONS 80

7. RECOMMENDATIONS 81

REFERENCES CITED $\quad 82$

$\begin{array}{ll}\text { APPENDIX I } & 87\end{array}$

$\begin{array}{ll}\text { APPENDIX II } & 96\end{array}$

$\begin{array}{ll}\text { APPENDIX III } & 106\end{array}$

$\begin{array}{ll}\text { APPENDIX IV } & 110\end{array}$

$\begin{array}{ll}\text { VITA } & 117\end{array}$ 


\begin{abstract}
Age-related macular degeneration (ARMD) and retinitis pigmentosa (RP) are the two leading causes of blindness in the world today. Despite enormous efforts and advances in clinical treatment of eye diseases, there is no established method or cure of degenerative processes in the eye, such as ARMD and RP. In these disorders, the primary cause of blindness is due to the loss of photoreceptors, however, the remaining conductive neural pathways in the inner retina are still intact and functional. As a result, the University of Louisville in collaboration with the Center of Innovative Visual Rehabilitation at the Eye and Ear Infirmary of Harvard University is currently developing a microelectrode array for direct stimulation of the epiretinal surface of the eye. A major problem associated with implantation of the microfabricated device is the inability to secure the implant to the epiretinal surface. To address this issue, our group designed retinal microtacks to attach the microelectrode arrays to the inner surface of the eye.

Microtacks were successfully fabricated out of titanium and silicon using ultrahigh-precision micromachining and microfabrication methods, respectively. Metrology was performed to verify the accuracy of both fabrication methods. Insertion and retention force experiments were performed on each tack design in fiber reinforced synthetic rubber and porcine eye tissue.

Results show that the titanium microtack design required less insertion force and greater removal force than that of the other designs in the fiber reinforced synthetic rubber. The synthetic rubber experiments displayed repeatable results with minimal deviation. The porcine ocular tissue showed poor repeatability with high deviation across all microtack designs.
\end{abstract}




\section{LIST OF TABLES}

Table 1. Optimized machining variables for machining Ti microtacks......................... 41

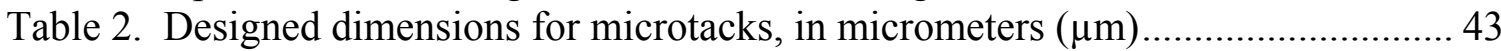

Table 3. Different theoretical values for buckling force for each microtack design using

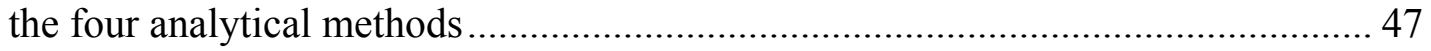

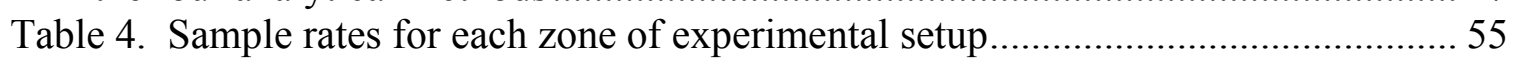

Table 5. List of designed, actual and percent difference of silicon pointed tip microtacks

Table 6. WYKO metrology data for the measured titanium microtacks ....................... 61

Table 7. Actual buckling failure corresponding to analytical data............................... 77

Table 8. Fiber reinforced synthetic rubber gasket material tensile tests to determine amount of tension applied to experiment samples ............................................. 103

Table 9. Maximum insertion and removal forces for each test sample of each microtack design in fiber reinforced synthetic rubber gasket material.................................. 111

Table 10. Displacement at maximum insertion and removal forces for each test sample of each microtack design in fiber reinforced synthetic rubber gasket material ...... 112

Table 11. Total insertion and removal forces for each test sample of each microtack design in fiber reinforced synthetic rubber gasket material................................. 113

Table 12. Maximum insertion and removal forces for each test sample of each microtack design in porcine eye tissue ....................................................................... 114

Table 13. Displacement at maximum insertion and removal forces for each test sample of each microtack design in porcine eye tissue................................................. 115

Table 14. Total insertion and removal forces for each test sample of each microtack design in porcine eye tissue .................................................................... 116 


\section{LIST OF FIGURES}

Figure 1. Cross-sectional view of retinal neural layers in fovea region and peripheral region. (Adapted from The Physiology Coloring Book, 1997) ................................. 4

Figure 2. Possible visual symptoms of ARMD (Obtained from

http://www.stlukeseye.com/Conditions/MacularDegeneration.asp) ....................... 10

Figure 3. . Example of RP visual symptom, loss of peripheral vision........................... 11

Figure 4. Neural pathway of the human brain. (Adapted from http://faculty.etsu.edu/currie/images/vision4.jpg) ............................................. 14

Figure 5. The cortical implant from the Dobelle Institute. (Obtained from

http://www.dobelle.com) ......................................................................... 15

Figure 6. Cross-sectional view of the placement of a subretinal implant in the eye ...... 16

Figure 7. Section view of the placement of an epiretinal implant in the eye................... 19

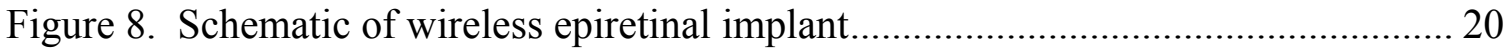

Figure 9. Preliminary epiretinal implant electrode design......................................... 21

Figure 10. SEM image of first silicon microtack., Shire et al. (2002)........................... 23

Figure 11. Ultra-High-Precision Micro Milling/Drilling Machine................................ 24

Figure 12. Images of micro clamp, a.) Solid Edge rendering, b.) machined micro-clamp

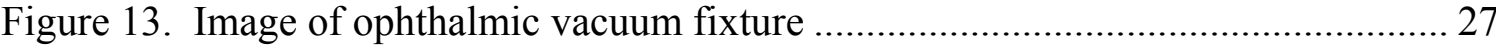

Figure 14. TA Instruments Rheometric Series RSA III.............................................. 28

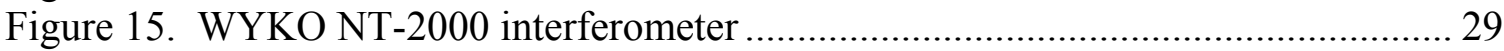

Figure 16. Zeiss SUPRATM 35VP Scanning Electron Microscope ............................. 30

Figure 17. Solid Edge rendering of three microtack designs: a) silicon chisel tip, ........ 32

Figure 18. Microfabrication processes for the chisel tip microtack design ................... 34

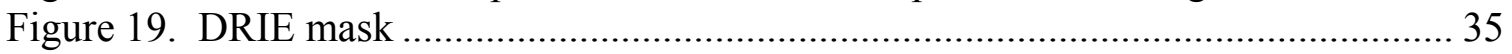

Figure 20. Microfabrication processes for the pointed tip microtack design ................. 36

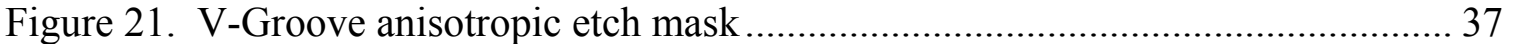

Figure 22. Schematic of bonding process for titanium foil ........................................ 38

Figure 23. Solid Edge rendering of the radial array design for machining of the

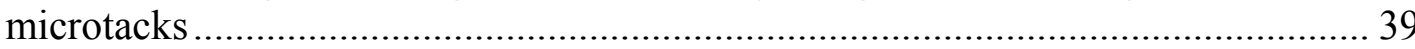

Figure 24. Image of the radial array of microtacks machined in a block of Lexan ${ }^{\circledR}$ using

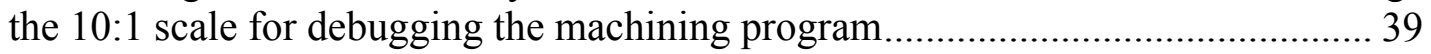

Figure 25. SEM image of 150 micrometer endmill ................................................... 40

Figure 26. Image of machining process using 150 micrometer endmill in Ti............... 41

Figure 27. SEM image of Ti microtack with burrs and machining flaws...................... 42

Figure 28. 2D sketch displaying the top and side views of the overall and critical dimensions measured for the microtack designs ............................................ 43

Figure 29. Clamped-Free end condition for buckling.............................................. 44

Figure 30. Comparison of microtacks for a: a) simply loaded column; b) titanium sharp

tip; c) silicon pointed tip; and, d) silicon chisel tip.............................................. 44

Figure 31. Prepared gasket material alongside vacuum fixture .................................... 48

Figure 32. Gasket material mounted under tension over the vacuum fixture ................. 49

Figure 33. Synthetic rubber sample placed in the RSA III system .............................. 51

Figure 34. Series of images from force measurement experiment, ............................. 52

Figure 35. Overall test setup for insertion and retention force experiments in the eyes.. 53 
Figure 36. Close up of vacuum fixture holding a porcine eye ........................................ 53

Figure 37. Typical placement of tack for insertion........................................................ 54

Figure 38. Insertion of microtack demonstrating tissue compliance issues..................... 54

Figure 39. SEM images of silicon chisel tip microtack ................................................. 57

Figure 40. SEM images of silicon pointed tip microtack ............................................ 58

Figure 41. a) Top view and b) 3D rendering of a silicon pointed tip microtack scanned

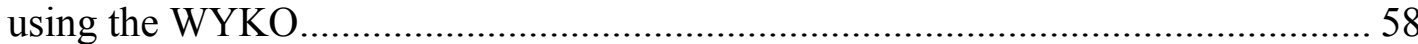

Figure 42. SEM images of machined and deburred titanium microtack ........................... 60

Figure 43. Typical Vertical Scanning Interferometer output image for a Titanium

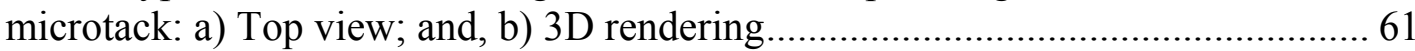

Figure 44. Typical normal force versus time plot for all microtacks tested in the fiber

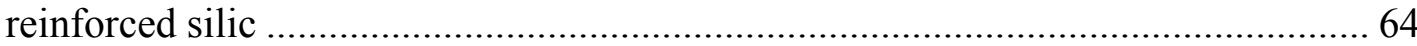

Figure 45. Average maximum insertion and removal forces for all microtack designs in the fiber reinforced silicone rubber gasket material. $\left({ }^{*} \alpha<<0.001\right)$......................... 65

Figure 46. Normal force versus displacement plot for all microtack designs in the fiber

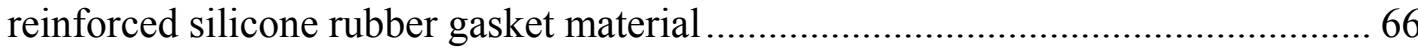

Figure 47. Average displacement required for insertion and removal for the microtacks

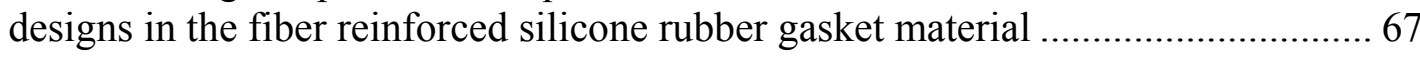

Figure 48. Normal force versus time plot illustrating how total force was calculated .... 68 Figure 49. Average total insertion and removal force for the microtacks designs in the

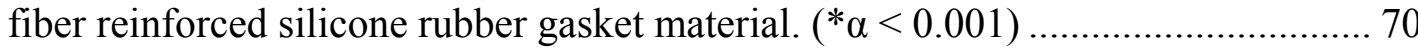

Figure 50. Stereomicroscope images of insertion/removal points of a) titanium, b) silicon pointed tip, and c) silicon chisel tip microtacks in the fiber reinforced synthetic

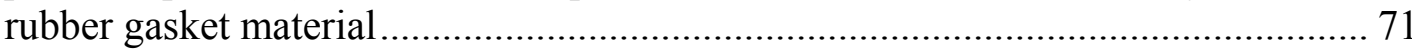

Figure 51. Average maximum insertion and removal forces for each microtack design in the porcine ocular tissue..................................................................................... 72

Figure 52. Average displacement required for insertion and removal of the different microtack designs in the porcine ocular tissue ....................................................... 73

Figure 53. Average total insertion and removal force for the microtacks designs in the

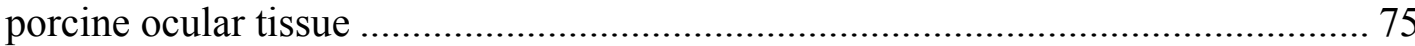

Figure 54. SEM image of the failure mode of the silicon chisel tip microtack ............... 78 Figure 55. SEM image of the failure mode of the silicon pointed tip microtack (Note: the tack was imaged upside down, hence the surface of the pointed tip is not shown.). 78

Figure 56. SEM image of the failure mode of the titanium microtack ............................ 79

Figure 57. Buckling criteria for titanium microtack .................................................... 97

Figure 58. Buckling criteria for silicon pointed tip microtack........................................ 98

Figure 59. Buckling criteria for silicon chisel tip microtack .......................................... 99

Figure 60. Set Transducer Characteristics window for transducer calibration on RSA3.

Figure 61. Transducer Calibration window ……………….................................... 101

Figure 62. Normal force versus time of tensile tests to determine amount of tension

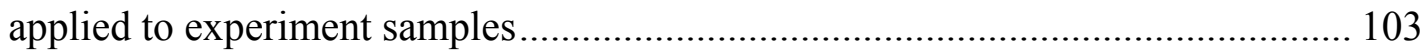

Figure 63. Screen shot of total force calculation using MathCAD ................................ 105

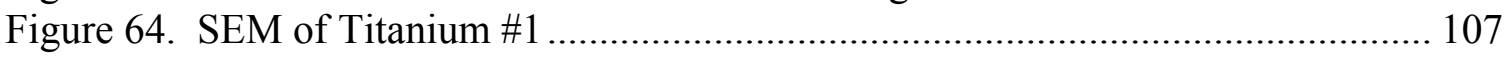

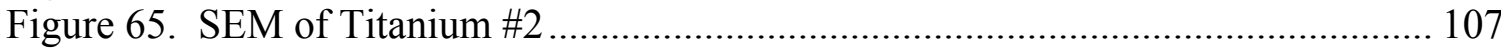

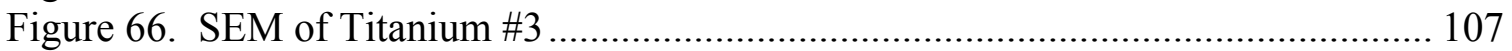




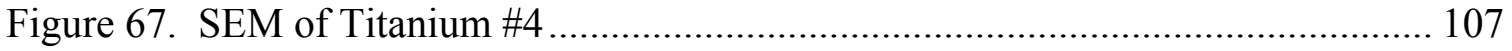

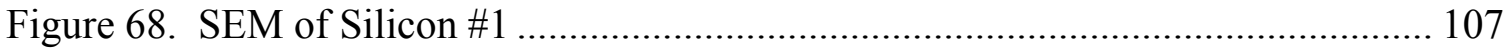

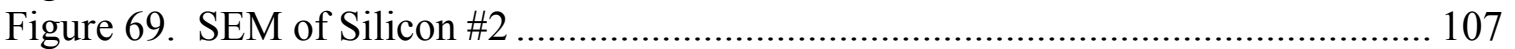

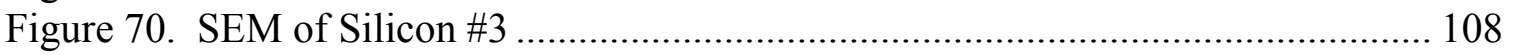

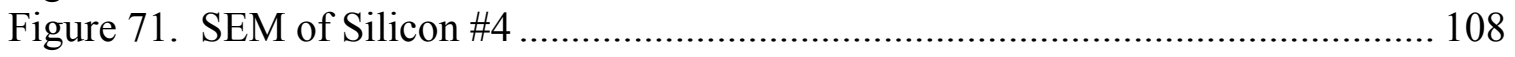

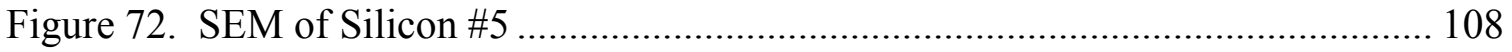

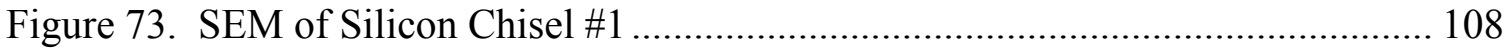

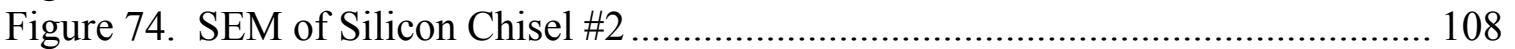

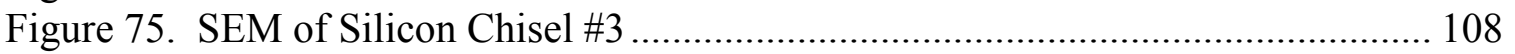

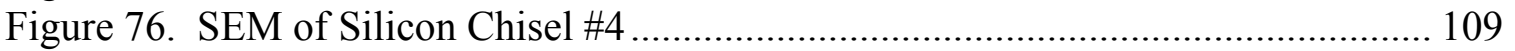

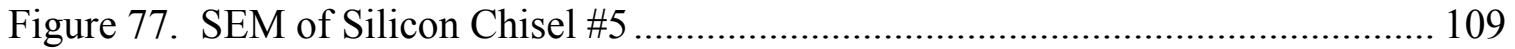

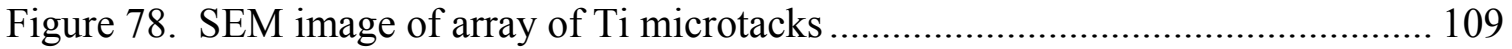




\section{INTRODUCTION}

\subsection{Purpose of the Study}

The purpose of this study is to develop alternative micromachining processes to fabricate retinal microtacks with 3-Dimensional features in biocompatible materials.

\subsection{Hypotheses}

- The 3-D microtack designs with the tapered tip point will require less insertion force than that of the planar microtack designs.

- For the tapered tip point designs, the 3-D titanium microtack will require less insertion force than that of the 3-D silicon microtack.

- The titanium microtack design with barbs will require greater removal force to extract the tack from a biomaterial compared to the silicon microtack designs.

\subsection{Significance of Study}

To this day, neither age-related macular degeneration (ARMD) nor retinitis pigmentosa (RP) have an effective treatment or cure. Artificial retinal implant designs are leading the way to restore sight to those who have been blinded by these diseases. Independent of the design, the ability to mechanically fasten a retinal prosthesis to the interior of the eye is a problem. Intraocular rotational motion can reach an angular velocity of more than 400 degrees per second [Humayun 2001 \& Margalit 2002]. Impulses such as these large velocities caused by the simple motion of the eye will require a sturdy device or structure to securely fasten any prosthesis slated for epiretinal attachment. In addition, silicon is a highly brittle material $\left(E=112.5 \mathrm{GPa}, \sigma_{y}=120 \mathrm{MPa}\right)$; thus, current silicon microtack designs are prone to breakage during insertion into tissue. Thus, alternative designs and different materials need to be investigated. 


\section{LITERATURE REVIEW}

\subsection{Background}

Age-related macular degeneration (ARMD) and retinitis pigmentosa (RP) are the two leading causes of blindness in the world today. Despite enormous efforts and advances in clinical treatment of eye diseases, there is no established method or cure for these degenerative processes. In these disorders, the primary cause of blindness is due to the loss or death of the rods and cones, which are the photoreceptors that convert light into electrical impulses; however, the remaining conductive neural pathways in the inner retina, e.g. the amacrine, horizontal, bipolar, and ganglion cells, as well as the optic nerve are still intact and functional [Medeiros 2001]. The University of Louisville Microfabrication Group in collaboration with the Center of Innovative Visual Rehabilitation Eye and Ear Infirmary of Harvard University is currently developing a microelectrode array for direct stimulation of the epiretinal surface of the eye [Rizzo 2001, Shire 2002]. A major problem associated with implantation of such a microfabricated device is the inability to secure the implant to the tissue surface. To address this issue, our group has designed retinal microtacks to physically attach the microelectrode arrays to the inner surface of the eye.

\subsection{Anatomy of the Human Eye}

The anatomy of the eye consists of several significant components that are vital to visual acuity, focus, sense of color, and the overall vision of the eye. Without the proper function of any of these anatomical features within the eye, vision can fade or be lost entirely. Some visual aids can be issued to assist in improving vision after the loss of 
some function, such as eye glasses, contact lenses, cortical prostheses or in the case of this study retinal prostheses. In recent years, retinal and cortical prostheses have been explored to address the loss or degeneration of the retina and its corresponding neural layers.

\subsubsection{Retina and its neural layers}

The retina is part of the nervous tunic and is a multi-layered sensory tissue that lines the back of the eye. It is analogous to a charge-coupled device (CCD) chip in a digital camera. Whereas a CCD chip contains millions of microphotodiodes, the retina contains millions of photoreceptors that capture light rays and convert them into electrical impulses that are then carried through the bipolar cell layer and transmitted to the ganglion cell region [Guyton 1996]. Axons of ganglion cells extend to the optic disk where they all exit as the optic nerve. The electrical impulses travel along the optic nerve to the brain where they are interpreted as images. The individual retina cellular layers and their functions are described below.

\subsubsection{Photoreceptors}

There are two types of photoreceptors in the retina: rods and cones. They are visual receptors highly specialized for stimulation by rays of light (Figure 1). The retina contains approximately 6 million cones that are specialized for color vision and sharpness of vision or visual acuity [Tortura 1997]. The majorities of these cones are contained within the macula and function best in bright light. Rods are specialized for vision in dim

light and also allow us to discriminate between different shades of dark and light and to 
see shapes and movement. There are approximately 125 million rods spread throughout the peripheral retina.

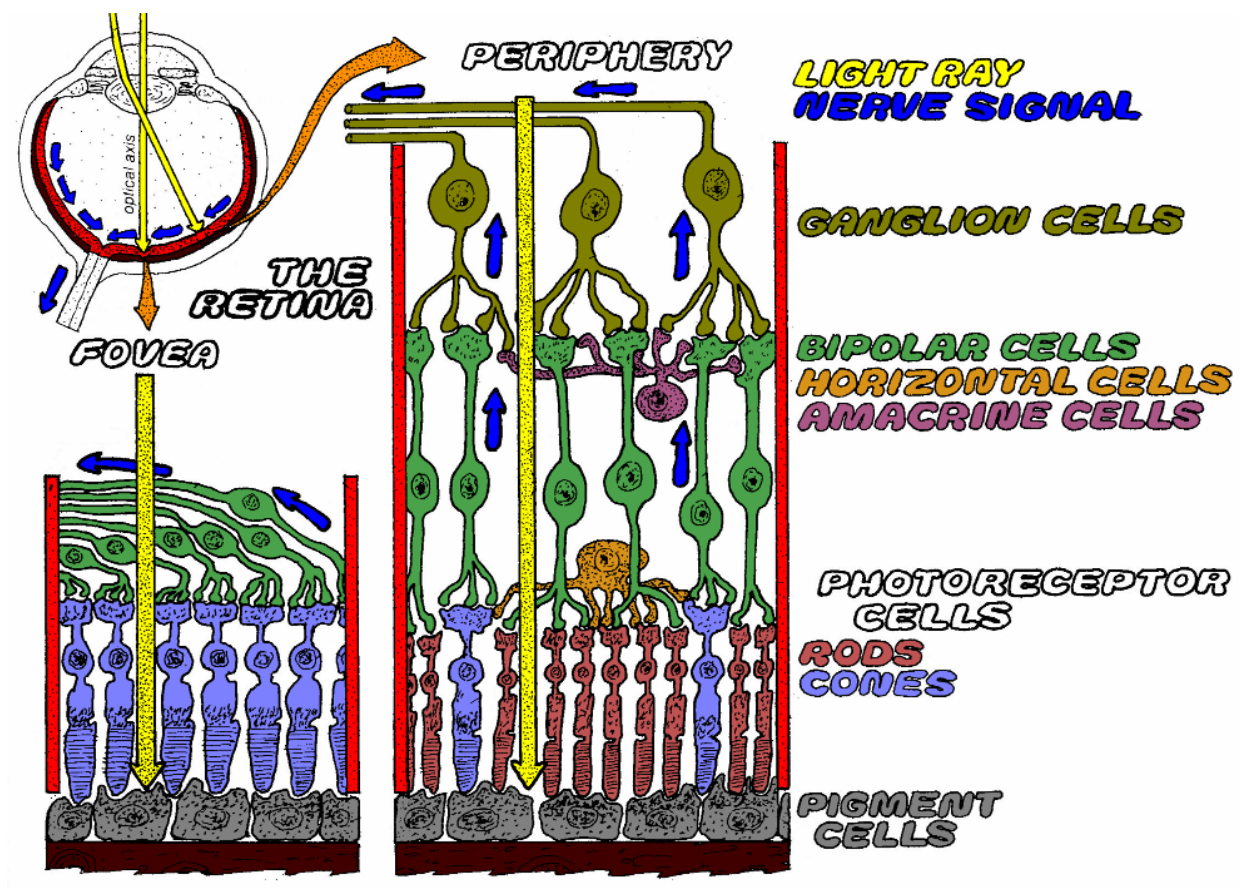

Figure 1. Cross-sectional view of retinal neural layers in fovea region and peripheral region. (Adapted from The Physiology Coloring Book, 1997)

\subsubsection{Bipolar Cells}

Bipolar cells are a class of retinal inter-neurons, named after their morphology, that receive input from the photoreceptors and send it to the ganglion cells. Bipolar cells are non-spiking neurons; their response to light is evenly graded, and shows lateral inhibition [Guyton 1996]. They are thus involved in the intermediate processing of light signals. Amacrine and horizontal cells are also considered intermediate bipolar processors, except they have no axons. The amacrine cells only interact with the ganglion cells while the horizontal cells only interact with the photoreceptor cells (Figure 1). 


\subsubsection{Ganglion Cells}

The retina contains more than 130 million rods and cones; yet the number of ganglion cells is a small percentage of that, only about 1.6 million. An average of 60 rods and 2 cones converge on each ganglion cell [Guyton 1996]. Axons of ganglion cells extend to the optic disk where they all exit as the optic nerve (Figure 1). These impulses travel along the optic nerve to the brain.

There are three groups of ganglion cells, named the W, X, and Y cells. Each ganglion cell type serves a specific function in the neuronal transfer of light into electrical signals [Guyton 1996].

- W-Cells

The W cell constitutes approximately 40 percent of the total ganglion cell population. They receive most of their excitation from the rods, and transmit through the bipolar cells. This ganglion cell type has a broad visual field due completely to the dendrites spread across a wider area. W cells are especially sensitive to the detection of directional movement anywhere in the visual field.

- X-Cells

The X cell, being the most abundant, constitutes approximately 55 percent of the total ganglion cell population. The $\mathrm{X}$ cell transmission is responsible for all color vision due to input received from at least one cone. The short dendrites of the $\mathrm{X}$ cells induce small fields which represent distinct retinal locations.

- Y-Cells 
The fewest of all types of ganglion cells, the $\mathrm{Y}$ cells fill the final 5 percent of the retinal population. They also have the broadest of dendritic fields among the three, visual signals are detected by the Y cells in widespread areas. This type of ganglion cell responds rapidly to either movement or light intensity.

\subsubsection{Macula}

The macula is the highly sensitive portion of the retina responsible for central vision and is located roughly in the center of the retina, temporal to the optic nerve. The macula allows a person to perform tasks that require central vision such as reading. It is approximately $6 \mathrm{~mm}$ in diameter and covers the central $21.5^{\circ}$ of visual angle [Jackson 2002]. The macula contains two sub regions: the fovea and parafovea. The fovea is the central region of the macula only $0.8 \mathrm{~mm}$ in diameter covering approximately $2.75^{\circ}$ of visual angle and is responsible for sharpness vision (Figure 1). It is in this region where there is a very high concentration of cones [Guyton 1996, Tortura 1997]. This visual acuity and spatial discrimination is primarily due to $\sim 1: 1$ ratio between the cones and ganglion cells. The parafovea encircles the fovea and is highly concentrated with rods. In young adults, rods outnumber cones in the macula by 9:1.

\subsubsection{Retinal Pigment Epithelium (RPE) and Bruch's Membrane (BM)}

The retinal pigment epithelium (RPE) and Bruch's Membrane (BM) are sustenance layers, acting as the nursing layers for the retinal cell layers (Figure 1). They sustain photoreceptor health by maintaining proper ionic balance, hydration, transportation and filtration of nutrients [Rowe-Rendleman 2003]. The RPE also 
replenishes photopigment that is bleached during light exposure. The BM acts as a support structure or basement membrane for the RPE and above the choroid [Anderson 2002].

\subsubsection{Choroid}

The choroid is sandwiched between the retinal layers and sclera. It is composed of layers of blood vessels that nourish the eye. It is a dark brown color to prevent any reflection of light within the interior cavity of the eye which would cause fowling of vision. The blood flow through the choroidal system, which nourishes the retina and RPE, are amongst the highest flow rates in the body [Jackson, 2002].

\subsubsection{Sclera}

The sclera is commonly known as the white of the eye. It is the tough, opaque tissue that serves as the eye's protective outer coating which covers approximately fivesixths of the posterior surface [Hecht 2003]. The sclera is continuous in the front of the eye with the cornea and in the back of the eye with the external sheath of the optic nerve.

\subsubsection{Conjunctiva}

The conjunctiva is the thin, transparent tissue that acts as a protective layer that covers the outer surface of the eye. It begins at the outer edge of the cornea, covering the visible part of the sclera, and lining the inside of the eyelids. The conjunctiva secretes oils and mucous that moistens and lubricates the eye. 


\subsection{Age Related Macular Degeneration}

Age-related macular degeneration (ARMD) is a degenerative condition of the macula. It is the most common cause of vision loss in the United States in people 50 years or older, and its occurrence increases with age [Tezel 2004, Ambati 2003, Jackson 2002, Humayun 1999, De l'Aune 2001, Stone 1992]. There are two forms of ARMD: Exudative (EXARMD) or wet form and Non-Exudative (NEARMD) or dry form. Both forms result in central vision deterioration.

EXARMD is the least common of the two types, accounting for approximately $15 \%$ of all ARMD patients diagnosed with the disease. It is the most destructive form, and is responsible for rapid and severe loss of vision due to the formation of choroidal neovascularization (CNV) [Tezel 2004, Medeiros 2001, Ambati 2003]. CNV occurs when new blood vessels from the choroid grow through the weakened BM, which is caused by RPE degeneration, reducing the amount of nourishment to the BM. The new blood vessels can leak or break in their adolescent state and newly formed hemorrhages lie between the RPE and BM, causing the RPE and retinal neural layers to swell inward. The gap between the retinal pigment epithelium and Bruch's membrane reduces or completely halts the transmission of blood to the photoreceptors and their by-products, resulting in the death of rods and cones in the area of the hemorrhage. In most patients, this can result in sudden loss of vision.

NEARMD is the more common of the two; approximately $85 \%$ of all ARMD patients are diagnosed with this form [Rowe-Rendleman 2003]. As the macular RPE degenerates and ceases to function properly, the photoreceptors become malnourished 
and gradually malfunction, resulting in a loss of central vision. RPE dysfunction also results in the formation of drusen. Drusen are amorphous yellow-white deposits found between the RPE and BM and are by-products of photoreceptor metabolism [Tezel 2004, Anderson 2002, Rapantxikos 2003]. With increasing age, drusen can become calcified or filled with cholesterol, appearing crystalline [Ambati 2003]. The size of the drusen typically are less than 63 microns, over time the drusen can regress in size, leaving weakened RPE tissue along with voids between the RPE and BM.

ARMD varies widely in severity. In the worst cases, it causes a complete loss of central vision, making everyday functions like reading or driving impossible. For others, it may only cause slight distortion. Fortunately, ARMD does not result in total blindness since it does not affect the peripheral vision. Both forms of ARMD can occur at the same time, producing symptoms of each.

\subsubsection{Symptoms of ARMD}

- Loss of central vision. This may be gradual for those with the dry form. Patients with the wet form may experience a sudden decrease of the central vision.

- Difficulty reading or performing tasks that require the ability to see detail.

- Distorted vision for wet form. Straight lines such as a doorway or the edge of a window may appear wavy or bent (Figure 2).

- Blurred vision for dry form. 


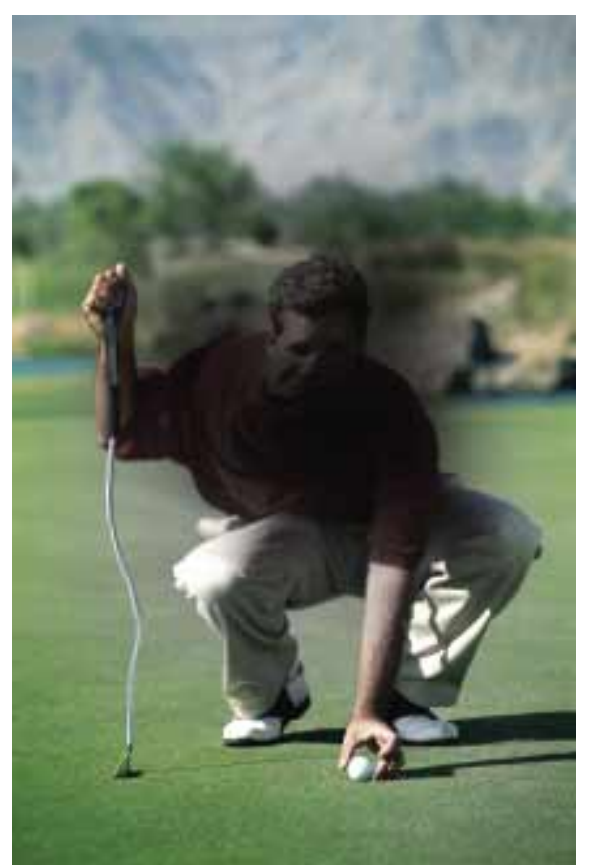

Figure 2. Possible visual symptoms of ARMD (Obtained from http://www.stlukeseye.com/Conditions/MacularDegeneration.asp)

\subsection{Retinitis Pigmentosa}

Retinitis pigmentosa (RP) is a rare, non-inflammatory hereditary disease that causes the rod photoreceptors in the retina to gradually degenerate; approximately 1 out of 3500 people is affected by this disease [Sharma 1999, Medeiros 2001, Gupta 2003]. The majority of the rod population is located near the periphery of the retina and is responsible for peripheral and night vision. Usually, patients with RP first notice difficulty seeing in dim light, such as difficulty with night vision, and gradually lose their peripheral vision. The course of RP varies. For some, the effect on vision may be mild. Others experience a progression of the disease that leads to blindness. Clinicians diagnose RP by examining the retina with an ophthalmoscope. The classic sign of RP is clumps of pigment in the peripheral retina called bone-spicules, which are spots on the peripheral retina that are relatively darker in color than that of the central macula region. 
In many cases, RP is diagnosed during childhood when the symptoms begin to become apparent. However, depending on the progression of the disease, it may not be detected until later in life.

\subsubsection{Symptoms of RP}

- Difficulty seeing in dim lighting or night vision

- Gradual loss of peripheral vision (tunnel vision, Figure 3)

- Glare

- Loss of contrast sensitivity

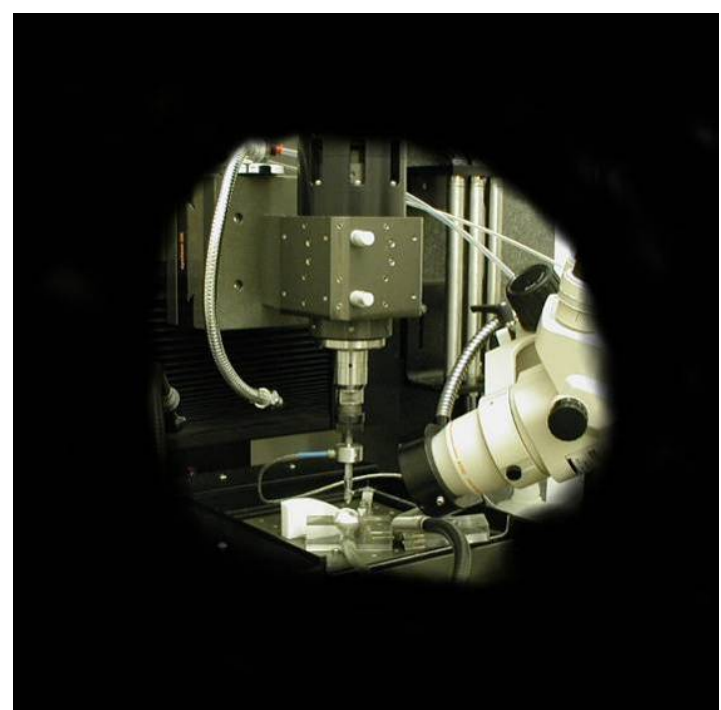

Figure 3. . Example of RP visual symptom, loss of peripheral vision

\subsection{Treatment Possibilities for ARMD and RP}

There are several treatment possibilities for ARMD. A high-dose vitamin and antioxidant treatment is the only proven treatment for slowing the progression of the degeneration process caused by both wet and dry forms of ARMD [Tezel 2004]. 
Currently, the goal for treating wet ARMD is the destruction and removal of the CNV. Below is a list of processes for $\mathrm{CNV}$ removal:

- Laser photocoagulation

- Photodynamic treatment

- Anti-Angiogenic Therapy

- Radiation

- Thermotherapy

At best, the above treatments yield scarring beneath the photoreceptor layer, leading to eventual receptor death and loss of vision. All target the removal of $\mathrm{CNV}$, ignoring the fact that the reformation of a healthy RPE is required to regain vision [Tezel 2004]. Treatments that do not change or heal the subretinal RPE interface only serve as a band aid and do not cure ARMD.

Like ARMD, retinitis pigmentosa has several treatment possibilities. Doses of vitamin A reduce the degeneration process [Sharma 1999]. Gene therapy, investigations into growth factors, and even a patient's diet have been determined to influence RP as well. To date, there is no cure to revitalize the lost photoreceptors and restore vision back to normal.

\subsection{Preservation of Ganglion Cells}

Although both age related macular degeneration and retinitis pigmentosa ultimately result in the loss of photoreceptors in the respective areas affected by the disease, the remaining bipolar and ganglion cell layers are moderately undamaged. In a study of pathological correlation by comparison, Medeiros et al. (2001) determined that 
in the case of wet ARMD, despite immense loss of photoreceptors, there was a $53 \%$ preservation of ganglion cells in the end stage of the disease. In the case of dry ARMD, there is no significant loss of ganglion cells [Medeiros 2001]. There was less than a $10 \%$ loss of ganglion cells in 5 out of 6 eyes tested, compared to healthy eyes used as the control. As for RP, $30 \%$ to $75 \%$ of the ganglion cells survived in the macular regions compared to the control eyes [Stone 1992, Santos 1997, Humayun 1999]. The same eyes had a survival rate of $20 \%$ to $30 \%$ compared to the control eyes. Thus, with a sufficient number of ganglion cells being preserved in both diseases, electrical stimulation of the remaining neural retinal layers is a possibility for these patients to regain at least some vision.

\subsection{Current Visual Prosthetic Possibilities}

A visual prosthesis is a man-made device intended to partially restore or improve the vision of a person who has succumbed to diseases such as RP and ARMD. To help

these patients, visual prostheses are being developed for placement in the visual cortex of the brain for direct stimulation, or in the eye, either under the retina as a subretinal implant or on the retinal surface as an epiretinal implant, to excite the functioning optic nerve path. Each design possibility has certain advantages and disadvantages.

\subsubsection{Cortical Prosthesis}

The initial work towards a visual prosthetic was initiated with direct electrical stimulation of the visual cortex [Dobelle 2000]. While patients can lose their sight through degenerative processes such as RP, ARMD, or any other vision impairment 
disease, a cortical implant essentially bypasses the phototransduction performed by the retina; therefore, does not require any functionality of the eyes nor the optic nerves, which travel from the eyes through the optic chiasm to the visual cortex (Figure 4).

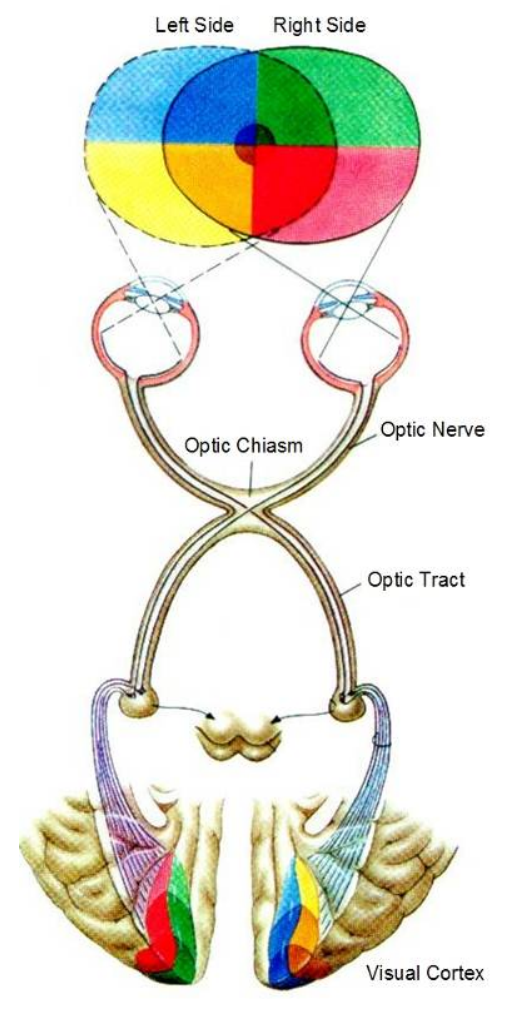

Figure 4. Neural pathway of the human brain. (Adapted from http://faculty.etsu.edu/currie/images/vision4.jpg)

Cortical stimulation requires placement of a microelectrode array into the visual cortex. The electrical stimulation produces one to four closely spaced phosphenes from each electrode [Dobelle 2000]. A phosphene is a sensation of light caused by excitation of the retina or visual cortex by mechanical or electrical means rather than by light [Hecht 2003]. The cortical implant from the Dobelle Institute (Portugal) Lda. consists of several components [Dobelle 2000]. A miniature video camera is mounted to a pair of special glasses which connects to a computer contained in a pack worn on the patient's waist (Figure 5). The computer interprets and simplifies the video via image pixilation 
and transmits it to the electrode array consisting of 64 individual electrodes in an 8 by 8 staggered array (Figure 5). The electrode array is implanted in the back of the head through a hole in the skull, which is drilled by the surgeon, directly on the visual cortex region of the brain. This type of visual implant has been successfully placed into eight human patients, each suffering from different types of blindness. Most cases were primarily from blindness due to traumatic accidents; however, the implant is applicable to virtually all causes of blindness. The device setup has been approved for commercial use in Europe.
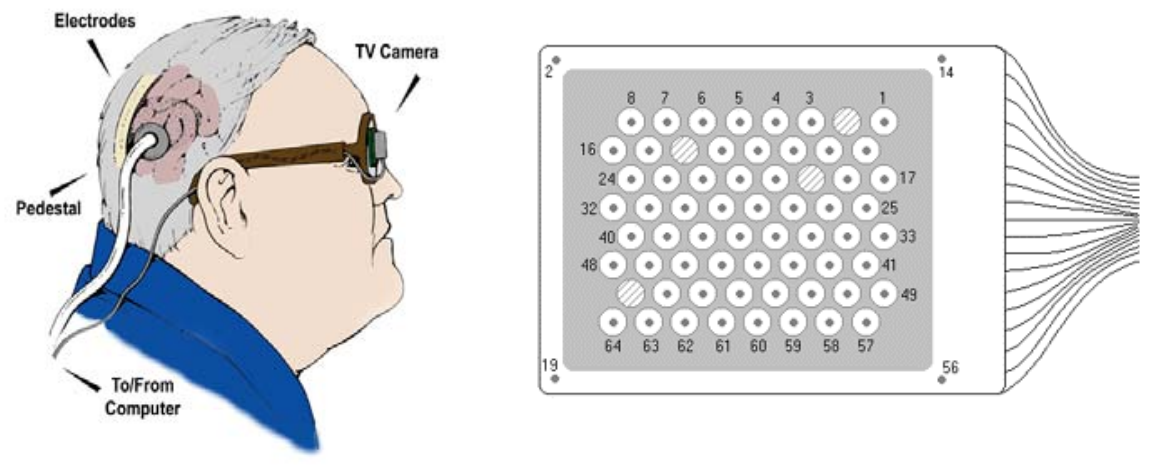

Figure 5. The cortical implant from the Dobelle Institute. (Obtained from http://www.dobelle.com)

Seven out of 8 patients whom have successfully had the cortical prosthesis implanted noted changes in vision, including four who stated the ability to see color [Dobelle 2000]. Two patients were able to detect people in light colored clothing, and were also able to detect straight edges as small as a pencil. Both were capable of driving an automobile, albeit slowly. Another patient demonstrated these abilities ten minutes after the viewing camera was turned on. On the downside, the implantation procedure is basically a form of brain surgery, entailing a serious risk to the patient. This fact suggests that other 
possibilities of visual stimulation should be explored, especially ones with less risk of severe injury or death to the patient.

\subsubsection{Microphotodiode Array Implant}

The microphotodiode array prosthesis is placed subretinally, i.e. under the retina, or sandwiched between the retinal pigment epithelium and the retinal neural layer. The prosthesis is placed in the region where the photoreceptors have been lost (Figure 6). The subretinal approach is considered a direct physical replacement of the degenerated photoreceptors [Schubert 1999, Tassicker 1954, Michelson 1985, Chow 1990, Nisch 1999].

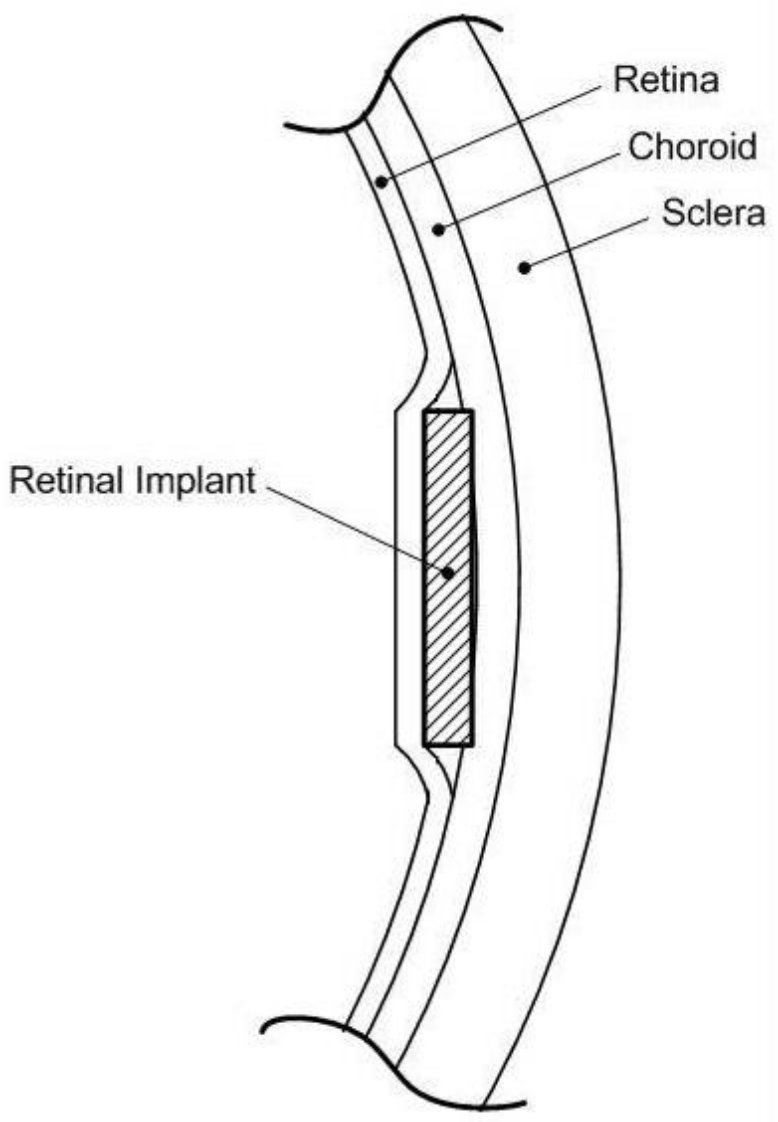

Figure 6. Cross-sectional view of the placement of a subretinal implant in the eye 
One type of subretinal prosthetic implant is an array of microphotodiodes which are used to power the implant and supply the stimulation to the electrodes for excitation of the remaining functional bipolar and ganglion cells of the retina in the general vicinity of the implant. These microphotodiodes convert light rays into electrical current, delivering potential changes correlating to changes in light intensity [Schubert 1999, Margalit 2002]. The microphotodiodes do not require any external power; they are solely powered by the incident light. Initially, the array consisted of a single microphotodiode with electrode however, currently, it ranges from 5000 to 7500 electrodes [Chow 1993, Peachey 1999, Zrenner 1999, Gekeler 2001]. Chow et al. (1993) and Zrenner et al. (1999) have separately developed their version of the subretinal implant, and both groups have created companies to investigate their respective designs, Optobionics Corporation and Retinal Implant AG, respectively. Both designs are quite similar, using microphotodiodes with a stimulating electrode in the middle of each. The prostheses are fabricated using traditional CMOS and microfabrication techniques. For both groups, the chip diameter ranges from 1 to $3 \mathrm{~mm}$ and has a thickness of approximately 25 to 50 microns. Both chip designs also have a span of approximately 30 microns between the 20 by 20 micron microphotodiodes, leaving a 10 micron gap between the photodiodes [Chow 1993, Zrenner 1999].

In Vivo experiments have been performed by both groups. Zrenner et al. (1999) have performed experiments in pigs and rabbits. The United States Food and Drug Administration authorized Optobionics Corporation to implant their subretinal chip design into 6 human patients that have lost vision due to retinitis pigmentosa, for a 2 year safety and feasibility study. Chow et al. [2004] reported that, at this time, no patients 
have shown signs of implant rejection, infection, inflammation, erosion, neovascularization, retinal detachment, or migration and most patients showed improved perception of brightness, contrast, color, movement, shape, resolution, and visual field size. However, the results of their study have not been duplicated by other investigators, so their results have come under close scrutiny.

The positioning of the subretinal implant is advantageous since the stimulating electrodes are inherently closer to the bipolar cells, which may lower the stimulation thresholds [Chow 1993, Zrenner 1999]. Subretinal implantation actually mimics physiologically the direction of phototransduction. Because the circular chip is sandwiched between the retinal neural layers and the choroid, it forms a virtually impermeable boundary for oxygen and nutrients to pass, causing malnourishment to the retina and potentially damaging or killing the already damaged bipolar and ganglion cell layers. One group has developed a procedure of drilling holes into the implant with a laser to prevent this possible barrier effect [Schubert 1999, Margalit 2002]. Currently, the subretinal design has much less sensitivity than naturally occurring photoreceptors. Normal ambient lighting conditions are at an approximate intensity level of 8 lux (lumen per square meter), the amount of light intensity needed to generate sufficient stimulation for the Chow and Zrenner devices are 12000 lux and 70000 lux, respectively [Maynard 2001]. More efficient diodes or active electronics requiring external power sources need to be developed for the subretinal approach to operate properly under normal or even dim lighting conditions. Thus, other implant approaches need to be developed. 


\subsubsection{Microelectrode Array}

The microelectrode approach is somewhat different from that of the microphotodiode approach. Specifically, the microelectrode arrays are typically placed on the inner surface of the eye, on top of the retina. It is analogous to placing a poster on a bulletin board, where the bulletin board is the retina and the poster is the microelectrode array (Figure 7).

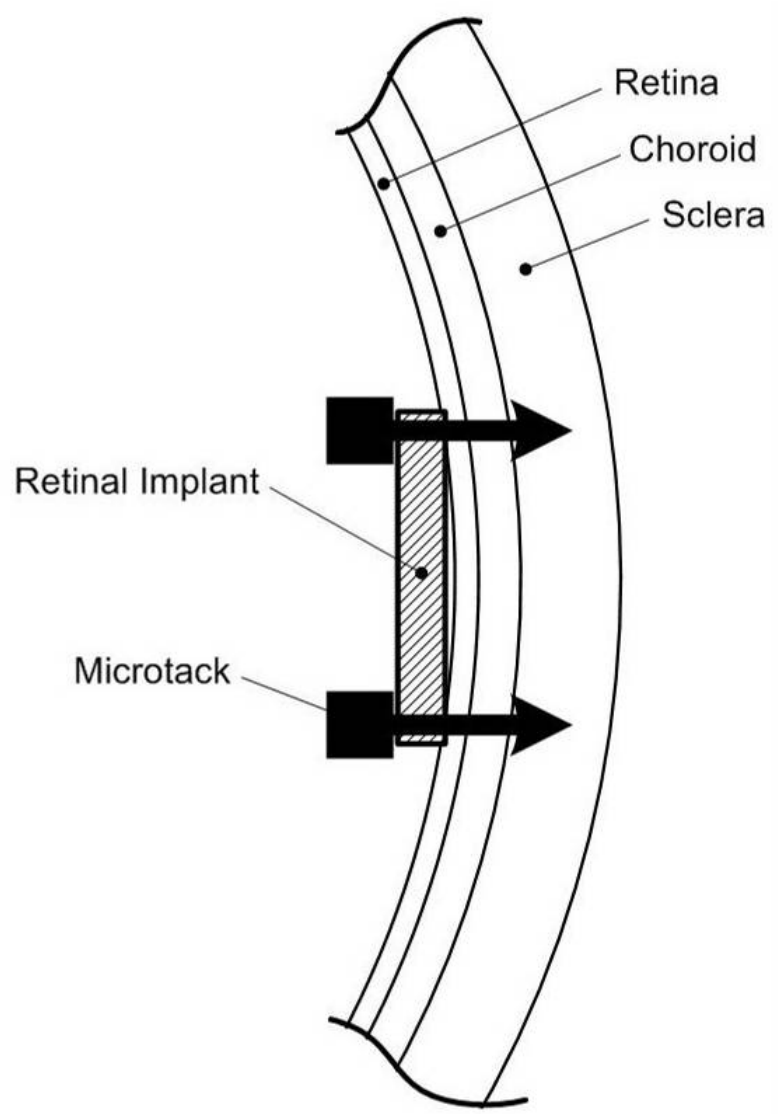

Figure 7. Section view of the placement of an epiretinal implant in the eye

There are a handful of groups that are developing and testing versions of a microelectrode array (MEA) device. All are using the same basic approach, which is an electrode array placed directly on the retinal surface [Rizzo 1997, Rizzo 2001, Meyer 2002]. Early designs began with a single electrode, while new arrays consist of 25 
electrodes. The electrodes stimulate the ganglion cells and their corresponding axons, instead of following the direction of phototransduction [Humayun 1999]. The cortical implant and epiretinal implant have their similarities whereas both devices require a high resolution CCD camera mounted to a pair of tinted glasses, a waist or belt mounted image processing unit that is connected to the electrode array. The ultimate goal in the microelectrode array project is to replace the belt mounted image processor with an implantable CMOS device, converting the wired setup into a wireless one, with a transmitter loop on the glasses and a receiver loop in the vitreous cavity of the eye (Figure 8).

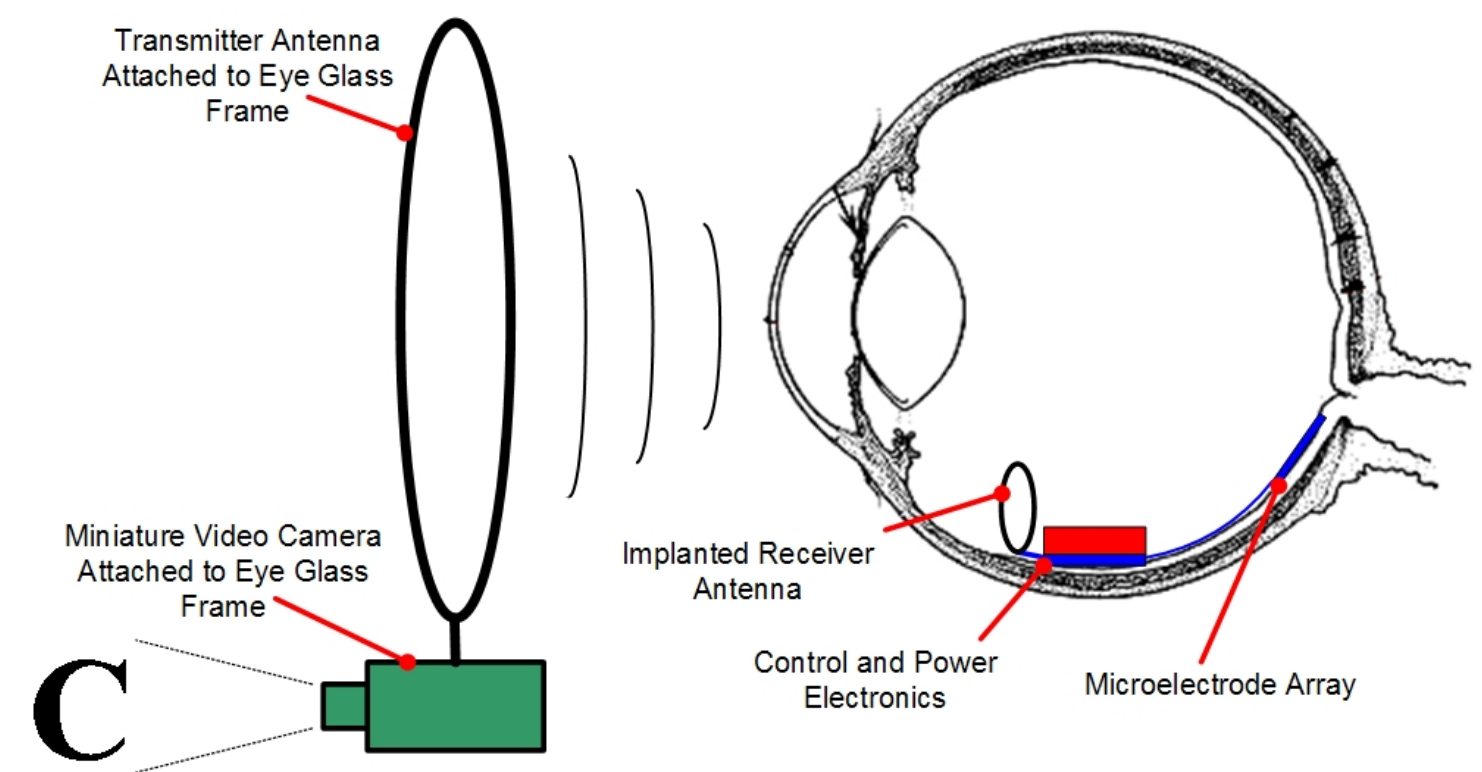

Figure 8. Schematic of wireless epiretinal implant

Two groups are leading the way in the MEA approach, Humayun et al. (2001) with Second Sight LLC, and Rizzo et al. (2001) with The Boston Retinal Implant Project. The designs of both groups have similarities. Rizzo et al. (2001), developed initial designs that contained a platinum electrode array encased in a thin film of polyimide, the 
array shown in Figure 9 contains 25 electrodes ( 5 by 5 array, ?? $\mathrm{mm}$ x ?? mm), whereas Humayun et al. (2001) has developed an implant that is fabricated using CMOS techniques and their first generation design consists of 16 electrodes ( 4 by 4 array, ?? mm $\mathrm{x}$ ?? mm). Both groups have been authorized by the USFDA to perform clinical trials for human implantation. Target patients will have been diagnosed with RP and ARMD, typically mild or end stage cases.

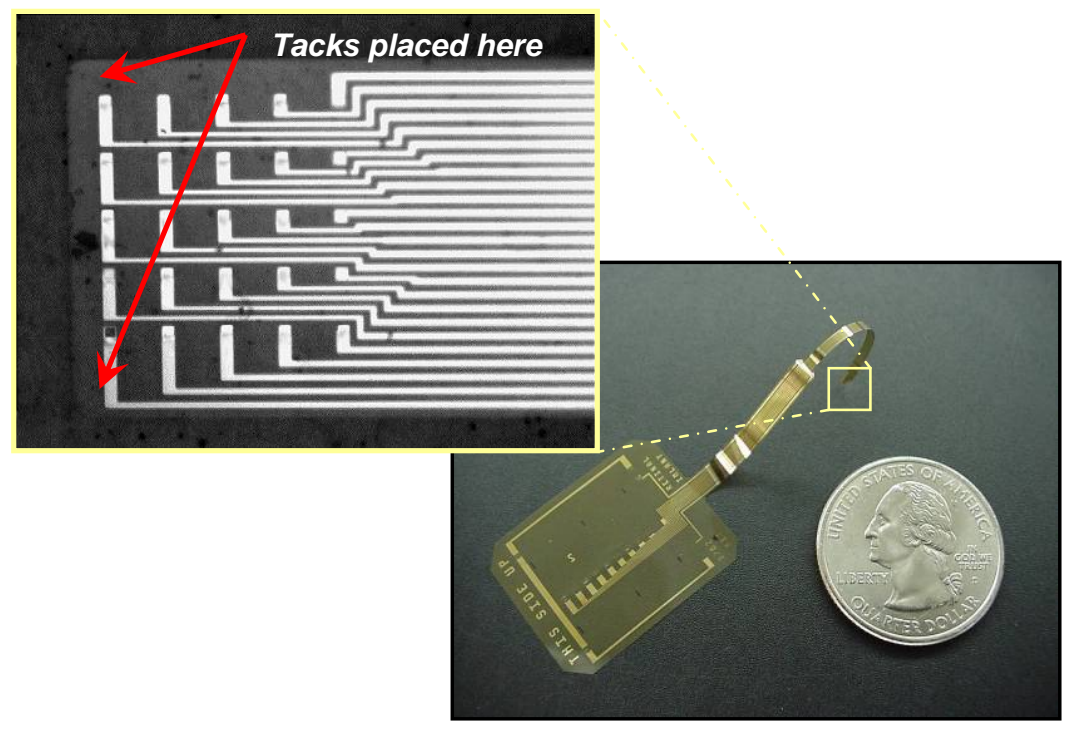

Figure 9. Preliminary epiretinal implant electrode design

\subsubsection{Fastening of MEA to Retinal Surface}

The ability to mechanically fasten the MEA prosthesis is a predicament. The intraocular rotational motion can reach an angular velocity of more than 400 degrees per second [Humayun 2001, Margalit 2002]. With this near impulse velocity created by the twitching motion of the eye, a sturdy device or structure will be needed to securely hold the prosthesis on the epiretinal surface. Several techniques for attaching the implant to the epiretinal surface were attempted in experiments by other groups, such as 
bioadhesives [Margalit 2002] and retinal tacks [Gerding 2001, Burke 1987, de Juan 1987, Abrams 1986, Puustjarvi 2001]. Bioadhesives used in the studies consisted of commercial fibrin sealants, UV curable glues, and hydrogels. The studies by Margalit et al., determined that one type of hydrogel, SS-PEG hydrogel (Shearwater Polymers Inc.) proved to be a strong adhesive and also nontoxic to the retina or other ocular tissue [Margalit 2000]. Bioadhesives do not allow easy replacement of the implant, possibly damaging retinal tissue under and around the adhered area. Whereas retinal tacks when removed for replacement may only damage the tissue at the removal site [Majji 1999].

Retinal tacks have been used in ocular surgeries for decades, most notably for the fixation of a detached retina. Having a resemblance to its household counterpart, the thumbtack, it is fairly large, with a head approximately 2 to $3 \mathrm{~mm}$ in diameter and a pin diameter of approximately $1 \mathrm{~mm}$ in diameter. These tacks are typically made of titanium, a highly biocompatible material that can either be used as an alloy or in its pure state [Brunette 2001, Barbucci 2002, Park 1992, Dee 2002]. This technique has been proven to be an effective approach for reattaching the retina, providing a permanent, stable and biocompatible form of adhesion [Gerding 2001, Burke 1987, de Juan 1987, Abrams 1986, Puustjarvi 2001]. Although these macroscale tacks have been successful in retinal reattachment, these tacks are too large for attaching MEAs to the epiretinal surface since the dimensions of the MEAs are approximately the size of the head of a single retinal tack, which is insufficient to securely fasten the MEA to the epiretinal surface. Thus, researchers have begun to design microscale tacks. 
The initial microtack designed for epiretinal attachment was developed by Doug Shire at Cornell University [Shire 2002], it was composed of silicon and had a minimal cross sectional area of approximately 100 square microns (Figure 10).

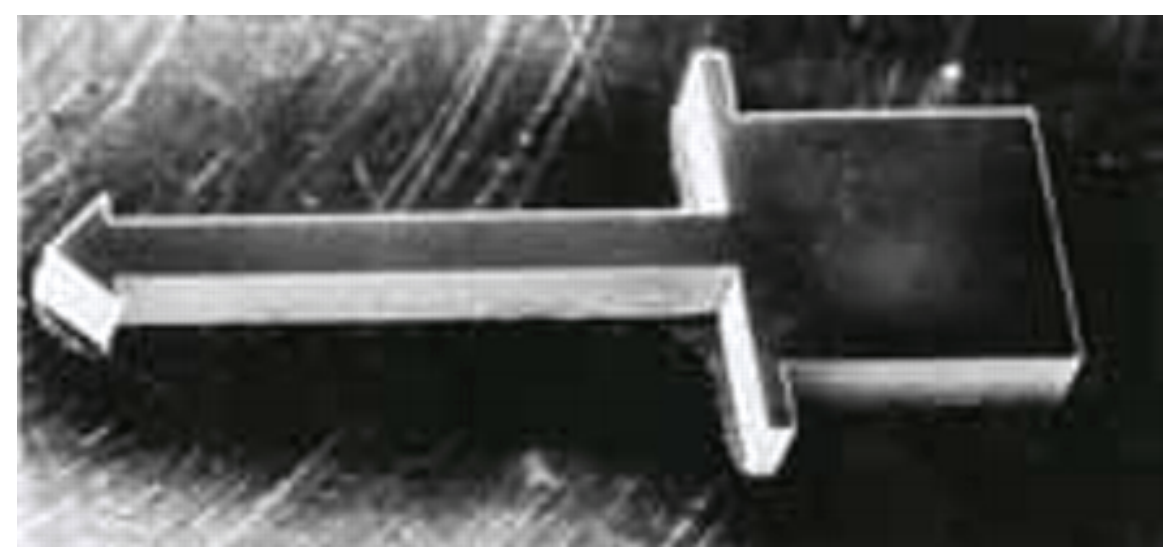

Figure 10. SEM image of first silicon microtack., Shire et al. (2002)

The ophthalmic surgeons had several problems inserting the initial designs of the silicon microtacks. Having such a small structural geometry induced failure of the microtack upon insertion into the epiretinal tissue. There were three basic modes of failure: 1) buckling of the relatively long, slender and brittle microtack shaft; 2) off-axis loading of the microtack imposed by the surgeon; and, 3) the micro-forceps slipping down the shaft of the microtack, ultimately breaking the microtack. Therefore, new microtack designs must be developed to increase the structural integrity during tissue insertion while minimizing the insertion force to reduce tissue damage. 


\section{INSTRUMENTATION AND EQUIPMENT}

\subsection{Ultra-High-Precision Micro Milling/Drilling Machine}

The Ultra-High-Precision Micro Milling/Drilling Machine (micromill) is a computer-numeric-controlled (CNC) milling station, using G-code, which is quite similar to those at the macro-scale (Figure 11). The micromill was exclusively developed by Craig Friedrich [Friedrich 1996, Vasile 1996] in collaboration with Dover Instruments Corporation (Westboro, Ma). The micromill at the University of Louisville is the newest of only three custom fabricated systems existing in the world; the other two are at Louisiana Tech (Ruston, LA) and Michigan Tech (Houghton, MI).

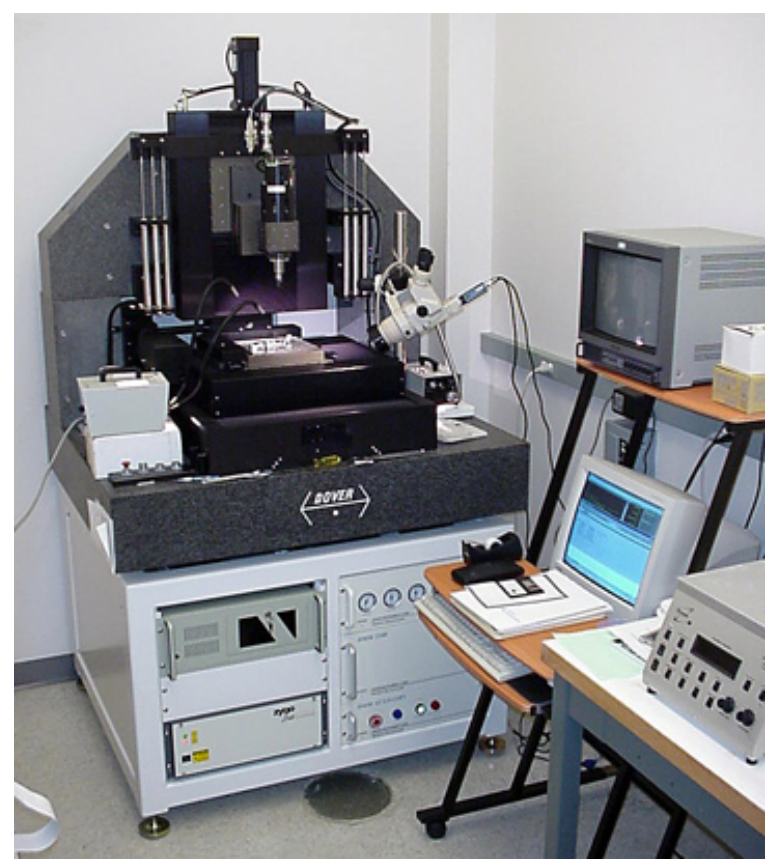

Figure 11. Ultra-High-Precision Micro Milling/Drilling Machine

The micromill is a four axis system, consisting of the $\mathrm{X}, \mathrm{Y}$, and $\mathrm{Z}$ translational directions of motion and the $\theta$ direction which represents the spindle rotation. All three of the translational and the rotational stages float on a thin cushion of air, or air bearings. 
Lubrication is not required for the bearings of this design, which minimizes the maintenance required for the system, and therefore increasing the accuracy. All that is needed for the bearings to function properly is clean, moisture and oil free air, regulated at 100 PSI. The absolute positions of the $\mathrm{X}$ and $\mathrm{Y}$ translational axes are controlled with a laser interferometry system (Zygo Corp, Middlefield, CT); having a highly accurate translational resolution of $1.25 \mathrm{~nm}$. Linear motors are used to actuate the $\mathrm{X}$ and $\mathrm{Y}$ axes. The $\mathrm{Z}$ axis consists of a brushless servo motor (Parker Hannifan Corp., Rohnert Park, CA) to turn a precision ground lead-screw (Universal Thread Grinding Co., Fairfield, CT). The rotation of the servo motor is translated into linear motion by the lead-screw. The absolute vertical position of the spindle is controlled using an exposed linear encoder (Heidenhain Corp., Shaumburg, IL) and a rotary encoder/resolver. The translational resolution of the $\mathrm{Z}$ axis is $20 \mathrm{~nm}$. All three translation axes have an overall travel of 150 $\mathrm{mm}$ ( $\sim 6$ inches). The spindle has a speed range that varies from $3 \mathrm{RPM}$ to a maximum speed of 20000 RPM.

There are two major concerns for mechanical machining at the micro-scale: 1) vibration from the surrounding environment; and, 2) thermal expansion/contraction of material due to temperature variations. To minimize the risk of vibration, four pneumatic cylinders are used to dampen and support a two ton granite block surface, in which all stages are mounted. The combination of the two techniques reduces the external vibrations to an absolute minimum. Holding the room to a constant ambient temperature and using cutting fluid or a misting fluid during machining procedures ensures that fluctuation in the temperature is also kept to a minimum. 
Machining is not the sole function of the micromill; it is a much more versatile machine. In the initial experiments, the micromill was used as a means for mounting a load cell and using the ultra-precise positioning capabilities for programmed input displacements. The adaptability of the micromill is only limited by the creativity of the user.

\subsection{Custom Fabricated Micro-Clamp}

Due to the size of the microtacks, a special fixture needed to be fabricated in order to properly secure the microtacks prior to testing. The custom-made micro-clamp allowed for firm grasp and proper orientation of the microtack for the force measurement experiments. The base or motionless portion of the micro-clamp (Figure 12), is comprised of 316 stainless steel and was manually machined in the UofL Mechanical Engineering Department machine shop. The retractable jaw (Figure 12) is made of 6061 aluminum and was machined on the micromill. The PMAC code that was written for the retractable jaw machining process is included in Appendix I. This clamp was used in the force measurement experiments in the RSAIII rheometric system to grip the microtack during microtack testing.

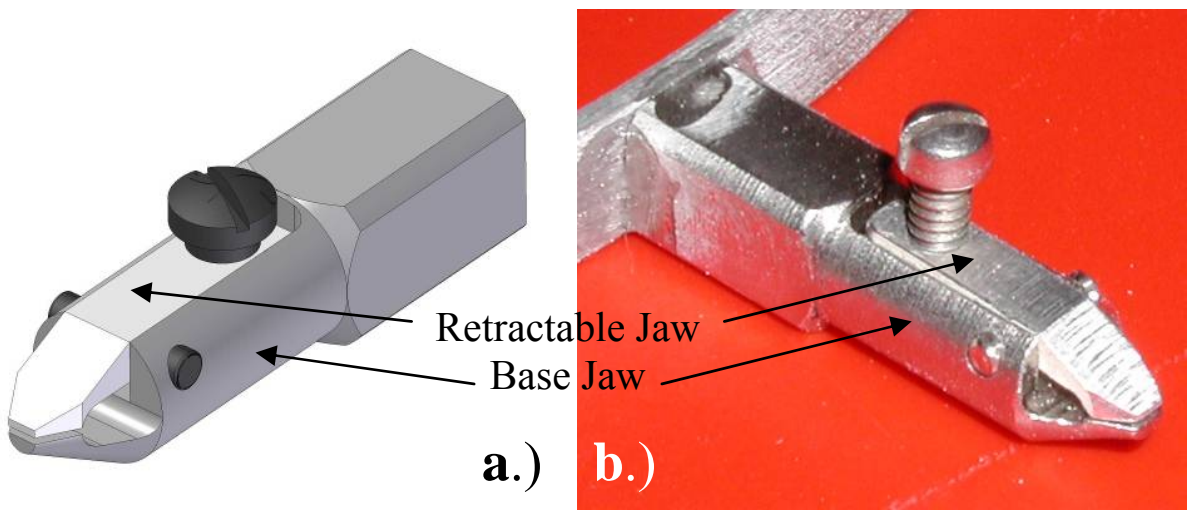

Figure 12. Images of micro clamp, a.) Solid Edge rendering, b.) machined micro-clamp 


\subsection{Ophthalmic Vacuum Fixture}

To securely grip the porcine eyes used in the force measurement experiments, a vacuum fixture was loaned from Dr. Henry Kaplan's group at the Department of Ophthalmology and Visual Sciences at the University of Louisville. The fixture ( $L=$ $75 \mathrm{~mm}, W=30 \mathrm{~mm}, H=50 \mathrm{~mm}$ ) was made of polymethylmethacrylate (PMMA), having a spherical cavity $(\mathrm{D}=25 \mathrm{~mm})$ to hold an eye ball (Figure 13). A vacuum line was drilled below the cavity allowing the eye to be partially pulled down, creating a secure and nondestructive grip on the eye.

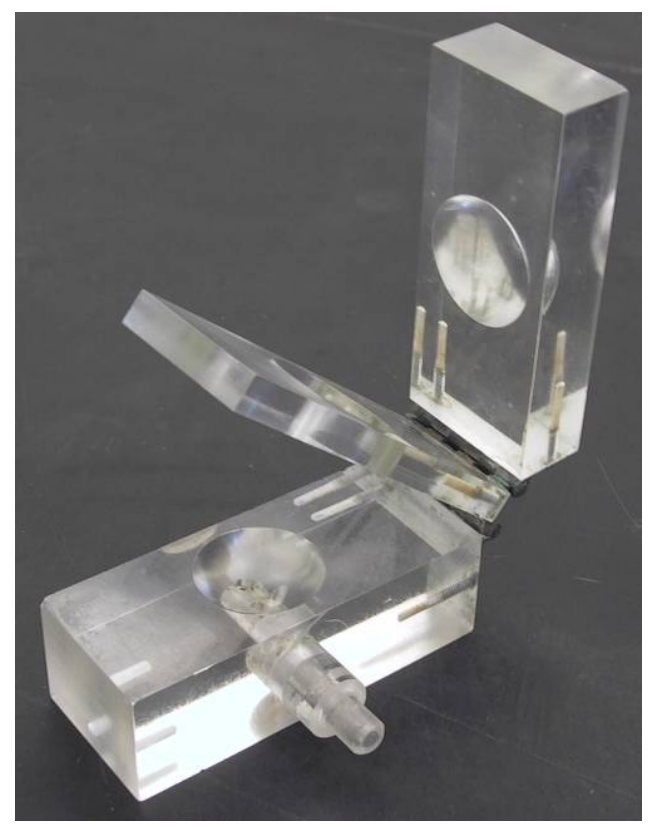

Figure 13. Image of ophthalmic vacuum fixture

\subsection{RSA III Rheometrics System Analyzer}

The RSA III Rheometrics System Analyzer (TA Instruments, New Castle, DE) tests the dynamic mechanical properties of solid materials by using a servo drive linear actuator to mechanically impose an oscillatory deformation, or strain, upon the material being tested (Figure 14). The sample is coupled between the motor and a transducer, 
which measures the resultant force generated by sample deformation [TA Instruments 2003]. Displacement and velocity are set by the operator, and the actual sample deformation is determined by the measured actuator and transducer displacement. The low load transducer, (Transducer \#1) has a maximum load of 367 grams and the high load transducer, (Transducer \#2) has a maximum load of 3670 grams. The system has an overall resolution of 0.100 grams.

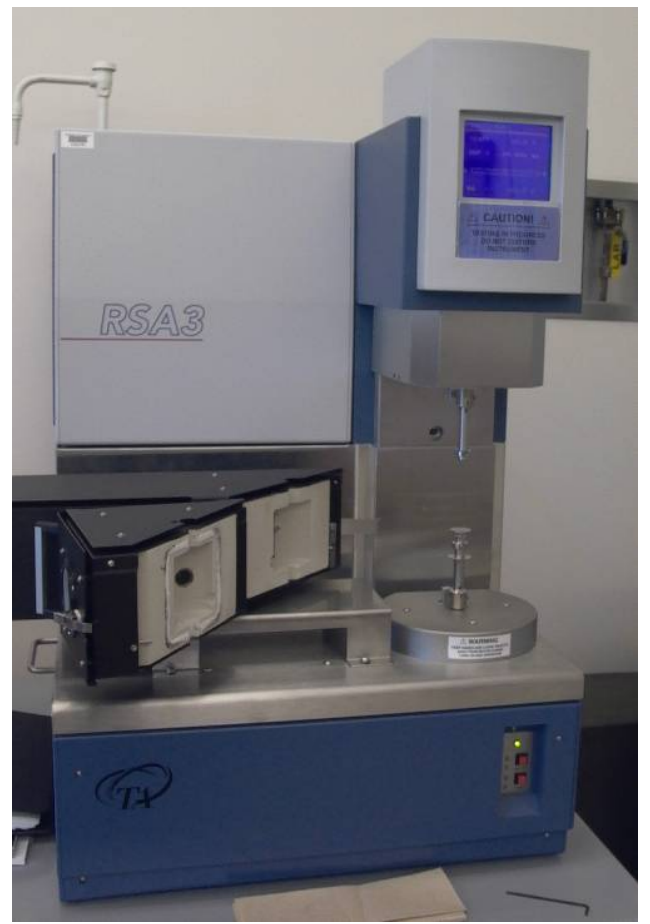

Figure 14. TA Instruments Rheometric Series RSA III

The RSA3 was used to accurately measure the load required to insert the different microtack designs and the load required to remove the microtacks from a synthetic material and actual biological eye tissue. The displacements and velocities were inputted into the computer interface and varied depending on material and microtack design. The recorded data was output as an ASCII text file and input into Microsoft Excel for analysis. 


\subsection{WYKO Metrology System}

The WYKO NT-2000 interferometer (Veeco Instruments, Woodbury, NY) takes data by combining the path of light reflecting off of a sample with the path of light reflecting off an internal reference surface (Figure 15). When the two paths combine, which occurs when alignment and focus of the test sample is satisfactory, the light waves interfere to produce a pattern of dark and light bands called fringes [Lichtenan 1997].

In vertical scanning interferometry (VSI) mode, the surface is profiled by scanning vertically downward so that each point on the surface produces an interference signal. At evenly spaced intervals during the scan, frames of interference data imaged by the video camera are captured and processed. Using a series of advanced computer algorithms, the system precisely locates the peak of the interference signal for each point on the surface and processes them to determine the surface height profile. The software program then calculates and displays the analysis output. VSI mode on the WYKO interferometer was used to perform metrology on all the fabricated microtacks. Overall and critical dimensions were recorded.

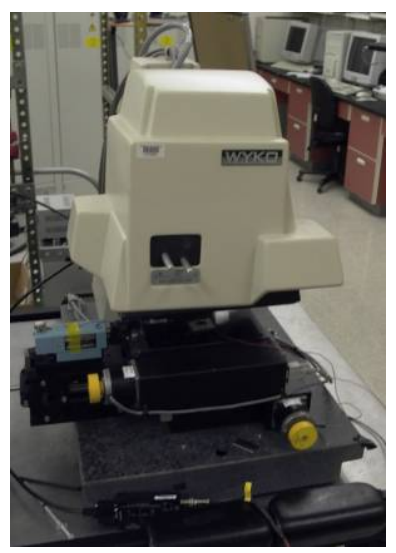

Figure 15. WYKO NT-2000 interferometer 


\subsection{Zeiss SUPRA ${ }^{\mathrm{TM}}$ 35VP Scanning Electron Microscope}

The SUPRA ${ }^{\mathrm{TM}}$ 35VP Scanning Electron Microscope (Carl Zeiss SMT Inc. Thornwood, NY) has a nominal resolution of 1.7 nanometers at $15 \mathrm{kV}$, and a nominal resolution of 2.0 nanometers at $30 \mathrm{kV}$ in variable pressure (VP) mode (Figure 16). It has a magnification range of $12 \mathrm{x}$ to $900,000 \mathrm{x}$, and a VP vacuum range of 2 to $133 \mathrm{~Pa}$ in steps of $1 \mathrm{~Pa}$. The Zeiss SUPRA ${ }^{\mathrm{TM}}$ 35VP scanning electron microscope was used to record true three dimensional and top view images of all fabricated microtacks.

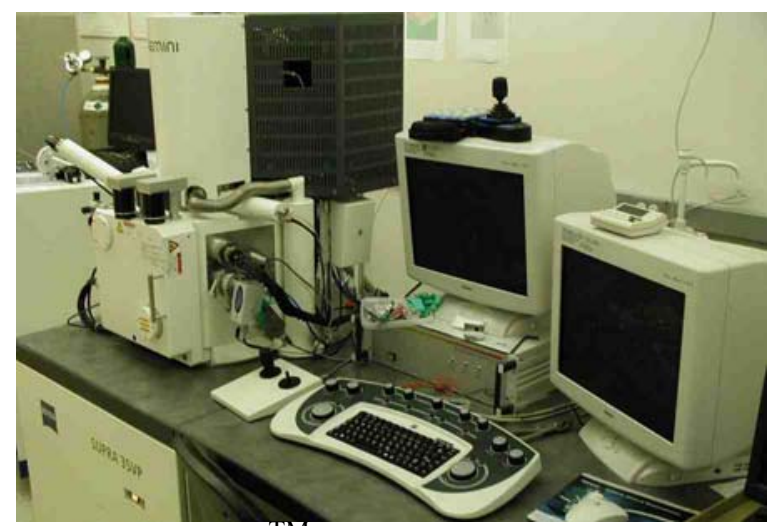

Figure 16. Zeiss SUPRA ${ }^{\mathrm{TM}}$ 35VP Scanning Electron Microscope

\subsection{SolidEdge Modeling Software}

SolidEdge V17 (UGS, Plato, TX) was implemented to generate three dimensional CAD models of the retinal microtack designs, fixtures and the microclamp components. This software assisted the user in determining proper geometric design constraints the microtack designs. 


\section{PROCEDURES}

\subsection{Description of Microtack Design}

There were three basic microtack designs investigated in this study, two of which were constructed out of silicon and the third machined in pure titanium. The most simple design of the three, the chisel tipped silicon microtack (Figure 17, a) is basically a two dimensional structure that is extruded in the direction normal to the mask. This microtack design is similar in geometry to that reported by Shire et al. (2002); however, it is a more robust design, with a larger shaft width and thickness, reducing its ability to buckle. To improve upon the chisel concept, a pointed tip was added to the design, which, theoretically, should reduce the amount of force required for insertion (Figure 17, b). Both silicon microtacks have the same straight barb geometry and "grip head" feature.

The third tack design, mechanically machined out of titanium, has a partially conical, tapered tip, which created a sharp point. Two curved barbs, similar to a fish hook, were added to increase the amount of retention force required to remove the tack, to prevent the microtack from "backing out" from the inner eye tissue (Figure 17, c). A modified "head" structure with two ledges and a step was also integrated into the new design to increase and enhance the gripping area for a pair of micro forceps during surgery, basically preventing the micro-forceps from slipping down the length of the tack and inducing failure in the tack or damaging retinal tissue. 


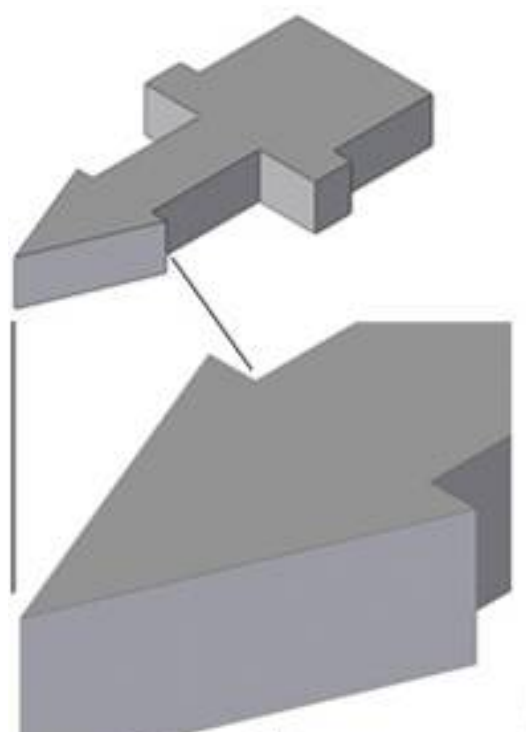

a.)

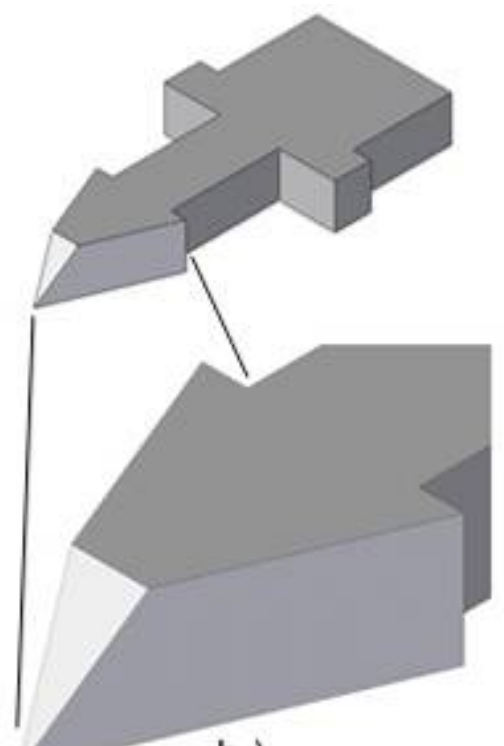

b.)

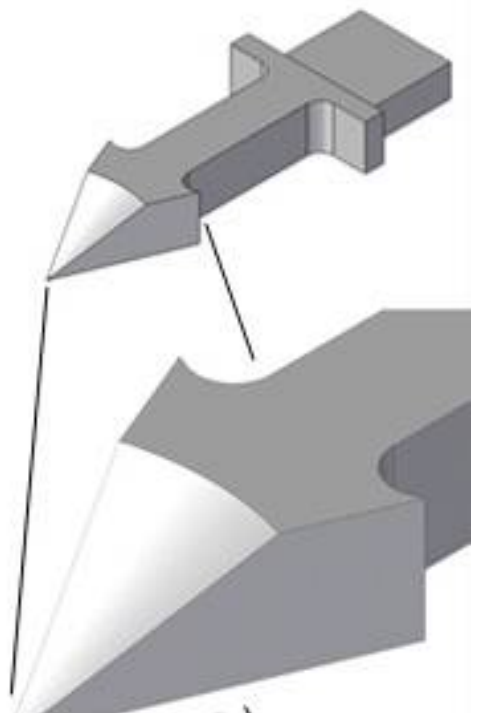

c.)

Figure 17. Solid Edge rendering of three microtack designs: a) silicon chisel tip,

b.) silicon pointed tip, c.) titanium sharp tip

\subsection{Microtack Fabrication}

Two different micromachining processes were used in the fabrication of the microtacks: 1) traditional silicon microfabrication techniques; and, 2) micro mechanical machining. The silicon microfabrication procedures (described in section 4.2.1) were performed by Dr. Jose Franco Sarabia while the micro mechanical machining procedures were performed by the author. The specific details of these fabrication techniques are described below.

\subsubsection{Silicon Microtack using DRIE and Microfabrication Techniques}

Deep Reactive Ion Etching (DRIE) in combination with silicon fusion bonding (SFB) and buried sacrificial oxide layers were utilized in forming the microtacks constructed in silicon. Both the chisel tip and pointed tip designs started with bonding a thermally oxidized "handle" wafer $(\mathrm{d}=100 \mathrm{~mm}, \mathrm{t}=500$ micrometers $)$ to a thermally 
oxidized $<100>$ silicon double side polished wafer $(\mathrm{d}=50 \mathrm{~mm}, \mathrm{t}=250$ micrometers $)$. The two wafers were bonded at room temperature and then annealed in nitrogen at $1000^{\circ} \mathrm{C}$ to complete the permanent silicon fusion bonding process (Figure 18, A). For the chisel tip design, a thick negative photoresist was first spun-on the device wafer (Figure 18 , B), photolithography was then performed on the top surface of the device wafer using a microtack mask similar to Figure 19. An oxide etch in buffered oxide etchant (BOE) was performed to open the windows for the DRIE process. The patterned oxide layer (Figure 18, C) functioned as a masking layer during the DRIE etch step. The device wafer was then anisotropically etched in an ICP (inductively coupled plasma) DRIE etch system (STS, Newport, United Kingdom) until the buried oxide layer was reached (Figure 18, D). The oxide layer on the handle wafer served as an effective etch stop during the DRIE process and also functioned as a subsequent release layer. The final step was to release the precisely etched silicon tack structures in hydrofluoric acid (HF) from the handle wafer (Figure 18, E). 


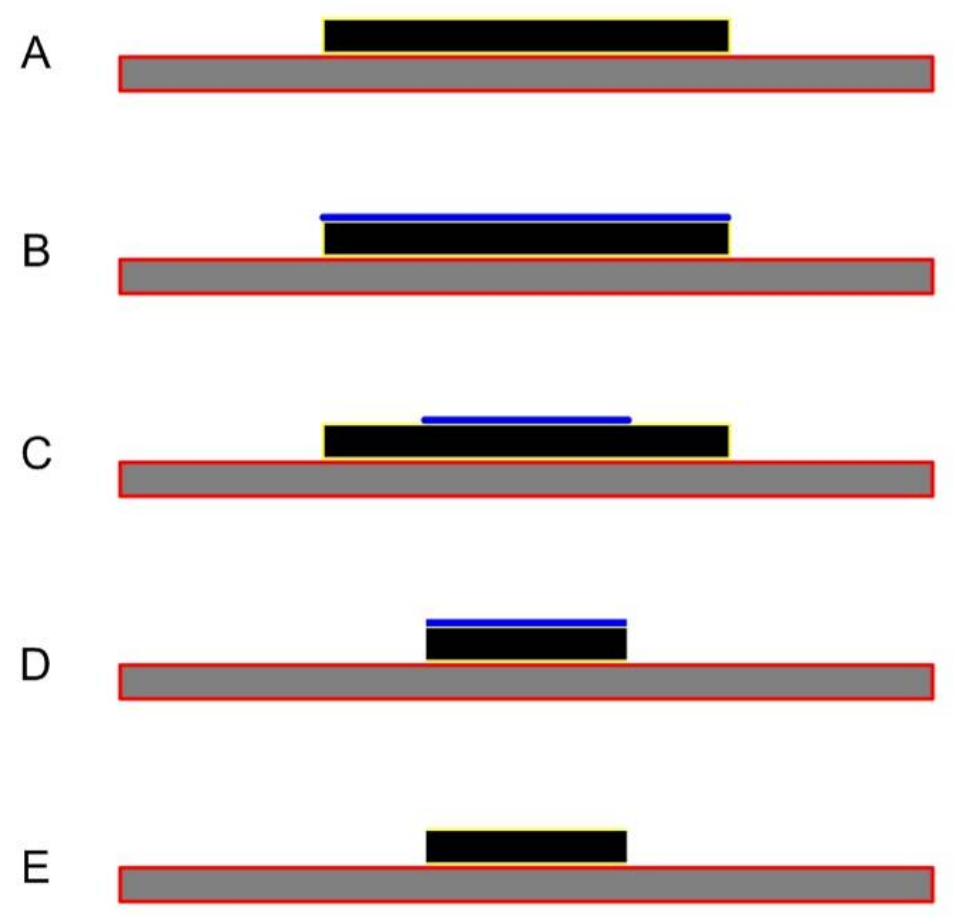

Figure 18. Microfabrication processes for the chisel tip microtack design 

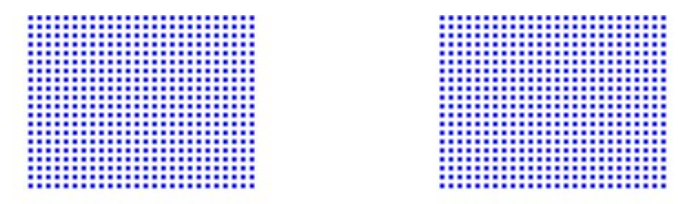

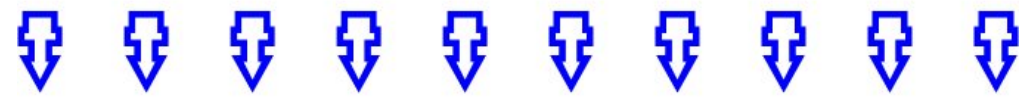

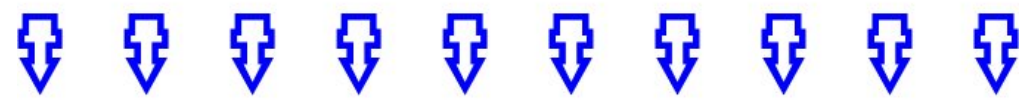

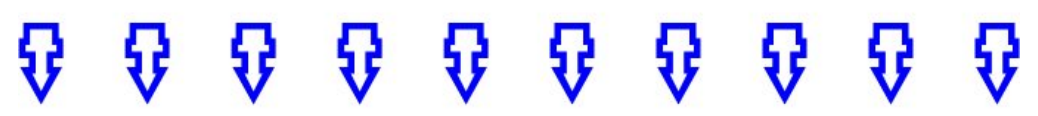

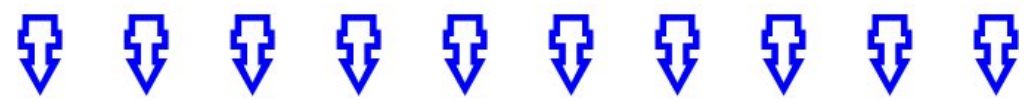

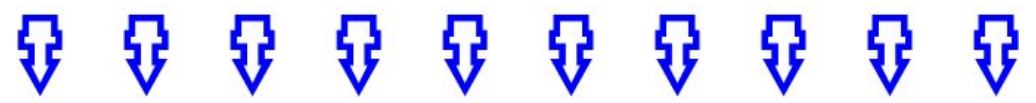

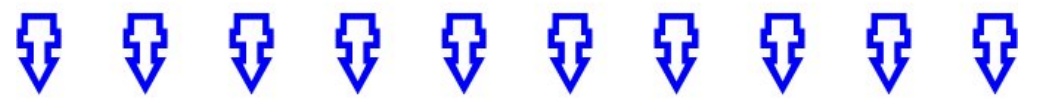
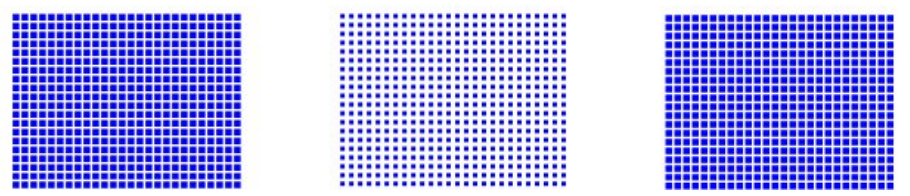

Figure 19. DRIE mask

To produce the pointed tip microtack design, the same SFB processes were performed as described above (Figure 20, A). Subsequently, a layer of positive resist was spun-on (Figure 20, B) and patterned (Figure 20, C) using the mask shown in Figure 21. To form the tip of the microtack, a V-groove was anisotropically etched into the device wafer (Figure 20, D), leaving a $54.74^{\circ} \mathrm{V}$-shaped tip. Photolithography is then performed on the top surface of the device wafer using a thick negative photoresist which functions as a masking layer during the DRIE etch step (Figure 20, E). The microtack mask in Figure 19 was then aligned to the existing V-grooves using the alignment markers and 
patterned as described above in the chisel tip section. The device wafer was then anisotropically etched in the DRIE etch system (Figure 20, F) and the microtacks were released from the handle wafer (Figure 20, G) using the same processing parameters as described above in the chisel tip section.

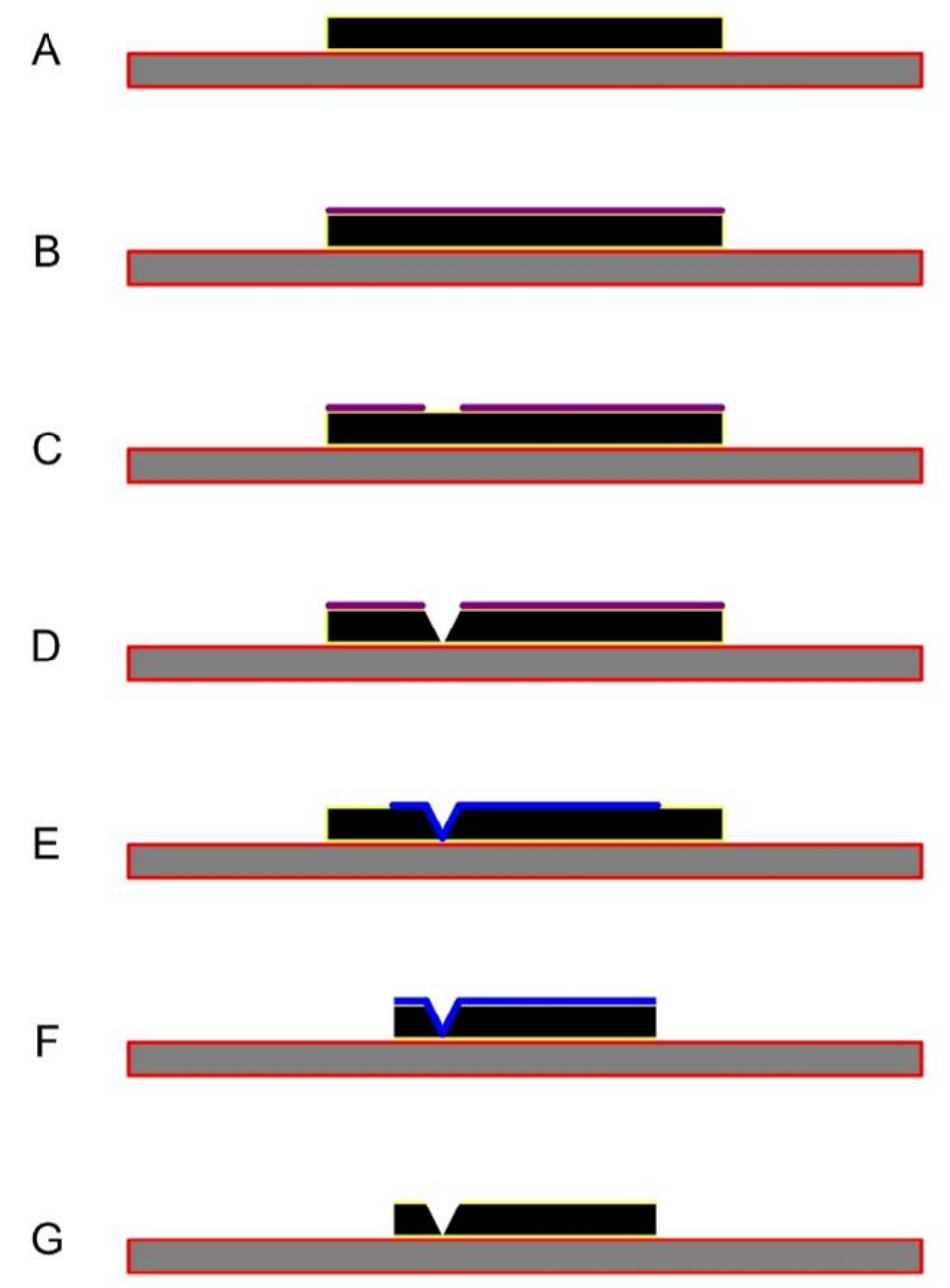

Figure 20. Microfabrication processes for the pointed tip microtack design 

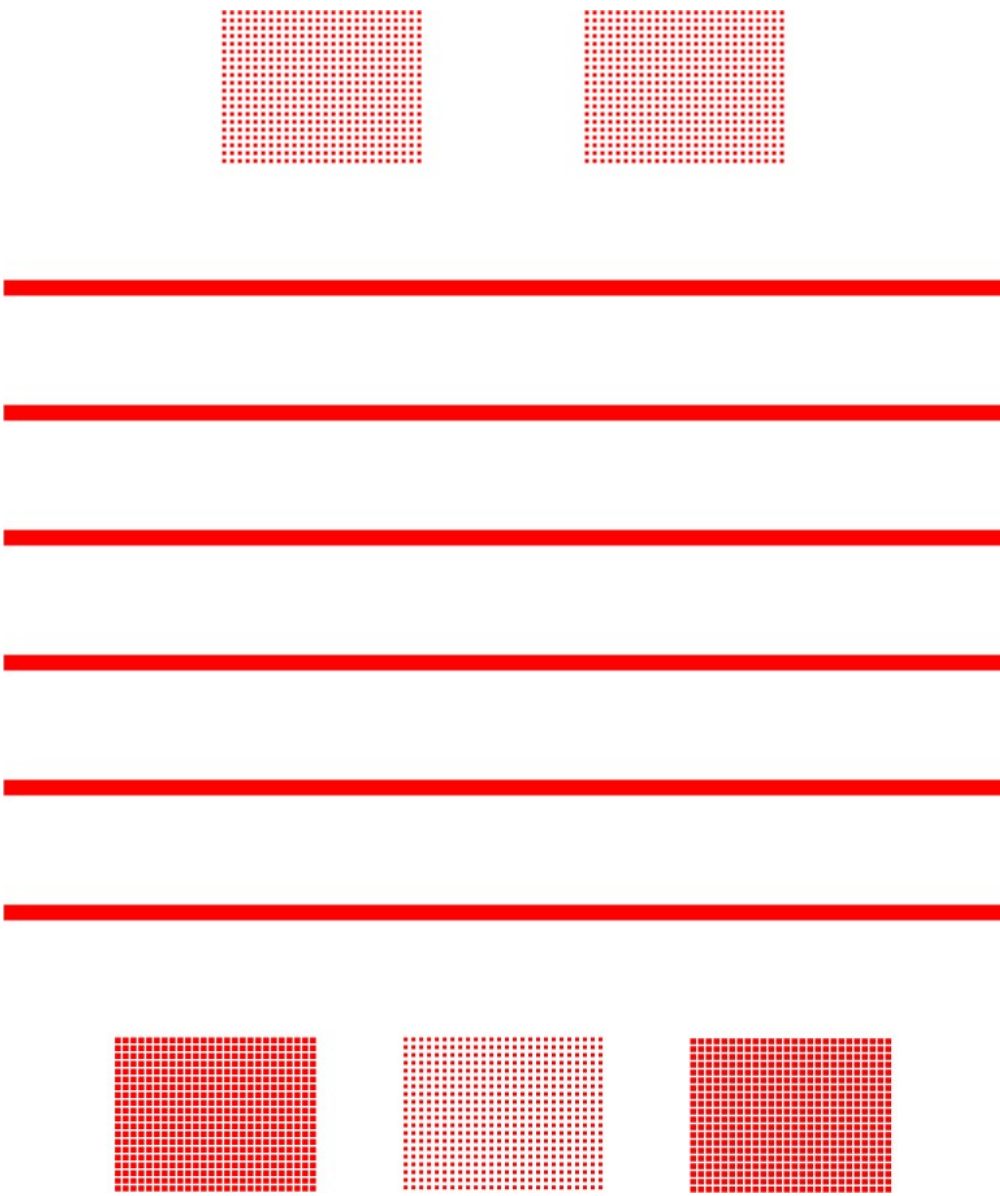

Figure 21. V-Groove anisotropic etch mask

\subsubsection{Sharp Tip 3-D Microtack Fabrication Technique}

Given the capabilities of the ultra-high-precision micromilling machine, a more ductile and biocompatible material, such as titanium, could be used in the micromechanical machining process [Cambron, 2003]. Prior to micromechanical machining, $25 \mathrm{~mm}$ square coupons were cut from a sheet of pure titanium foil (Alpha Aesar, Ward Hill, MA) with a thickness of 250 micrometers using a dicing saw (Disco, Manassas Park, VA). The Ti coupons were bonded and clamped using a 1 inch C-clamp (apply $5-10 \mathrm{lbs}$ of force) to a silicon wafer of similar size using a two part epoxy (88 Epoxy laminating resin \& 87 epoxy hardener, Fibre Glast Development Corp., 
Brookville, $\mathrm{OH}$ ) and allowed to cure in an oven at $65^{\circ} \mathrm{C}$ for approximately one hour (Figure 22).

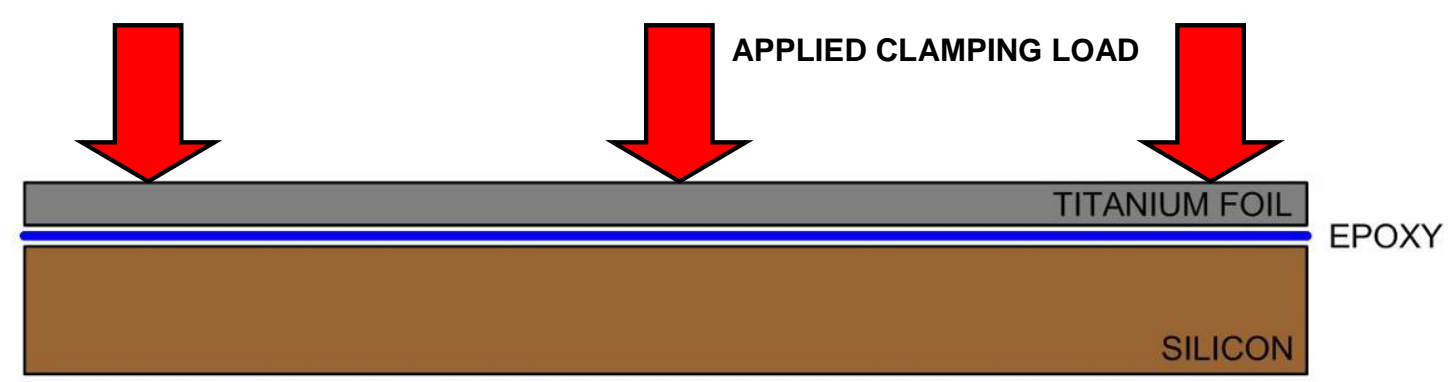

Figure 22. Schematic of bonding process for titanium foil

This procedure ensured flatness of the titanium substrate while increasing the precision of the micromachining processes by removing all gaps between titanium foil and silicon wafer and also protecting the milling stage from any damage resulting from the machining processes, when machining through the titanium the endmill will cut into the handle wafer instead of the milling stage. The thickness of the commercially available titanium foil provided the desired microtack thickness and enabled proper 3-D machining of the tack's sharpened tip, perimeter, and "grip head" for the micro-forceps.. Additionally, the versatility and ultra-high resolution of the $\mathrm{x}-, \mathrm{y}-$, and $\mathrm{z}$-translational stages of the micromilling machine allowed these new features to be incorporated into the updated design. Several machining algorithms were written and programmed into the milling machine motion controller using programmable multi axis controller (PMAC) code (Appendix I). Each microtack was machined serially, i.e. one tack at a time, around a radial array to reduce machining time (Figure 23). Initially, the machining program was written with an embedded scaling factor (10:1) to debug the program by machining a macroscale block ( $75 \mathrm{~mm}$ by $75 \mathrm{~mm}$ by $10 \mathrm{~mm}$ thick) of polycarbonate (Lexan $\AA$ ) using a $1.588 \mathrm{~mm}$ ( $0.0625 \mathrm{inch}$ ) two flute endmill (Figure 24). This debugging procedure saved 
time and money since the machining larger tack patterns enabled visual identification of machining errors and tools and substrate materials are both significantly cheaper than the 150 micron endmills and titanium material.

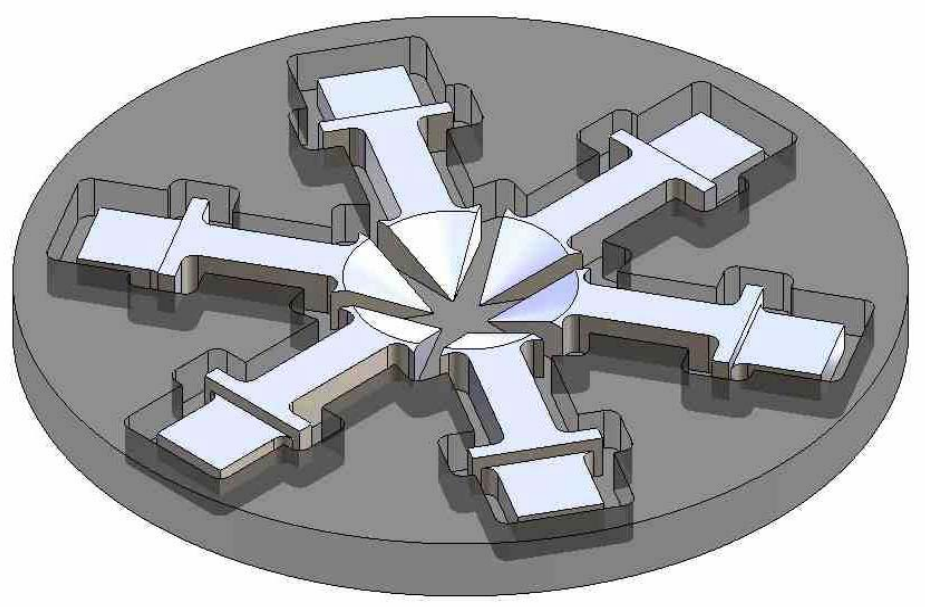

Figure 23. Solid Edge rendering of the radial array design for machining of the microtacks

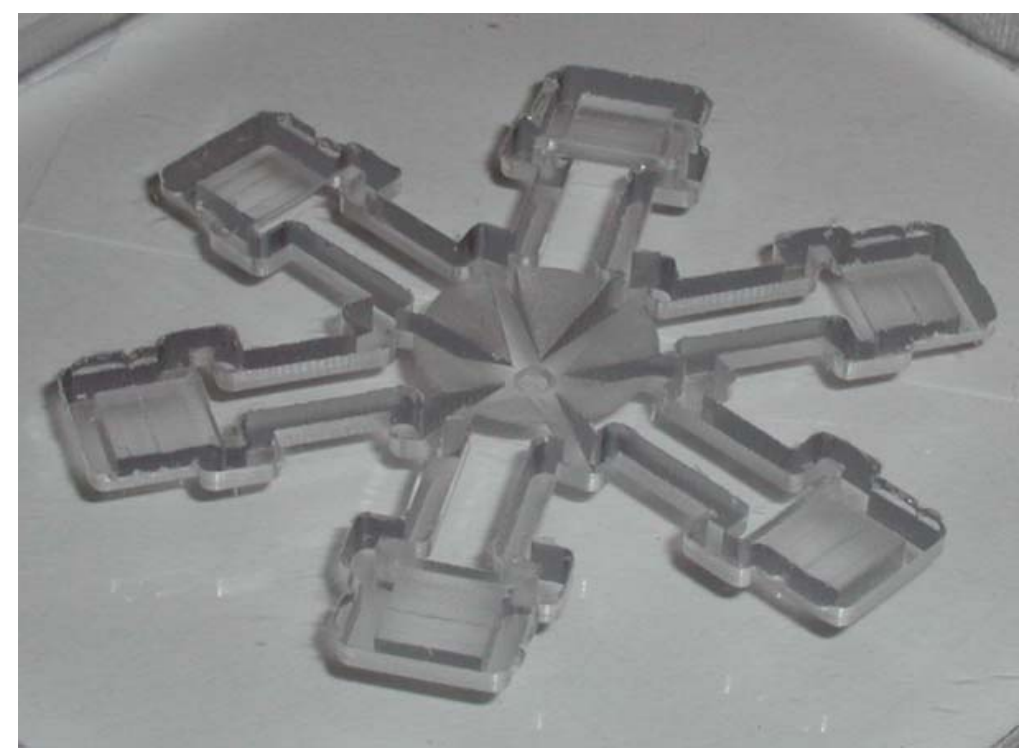

Figure 24. Image of the radial array of microtacks machined in a block of Lexan ${ }^{\circledR}$ using the 10:1 scale for debugging the machining program 
For micromachining the sharpened Ti tacks, a $2 \mathrm{~mm}$ standard jobber drill bit was used as bulk material removal of the pointed tip cavity (Figure 26). Then a 150 micrometer micro grain tungsten carbide end-mill (MiniTool Inc., Los Gatos, CA) was used to machine tacks at the final 1:1 scale (Figure 25). The endmill was relocated to the center of the microtack array and cut the perimeter, forceps grip and the pointed tip. The spindle speed, feed rate, plunge rate, depth of cut, and cutting fluid were characterized to determine the optimum machining process parameters (Table 1). An image of the actual machining process is shown in Figure 26. The machining time to complete a single microtack was approximately 25 minutes.

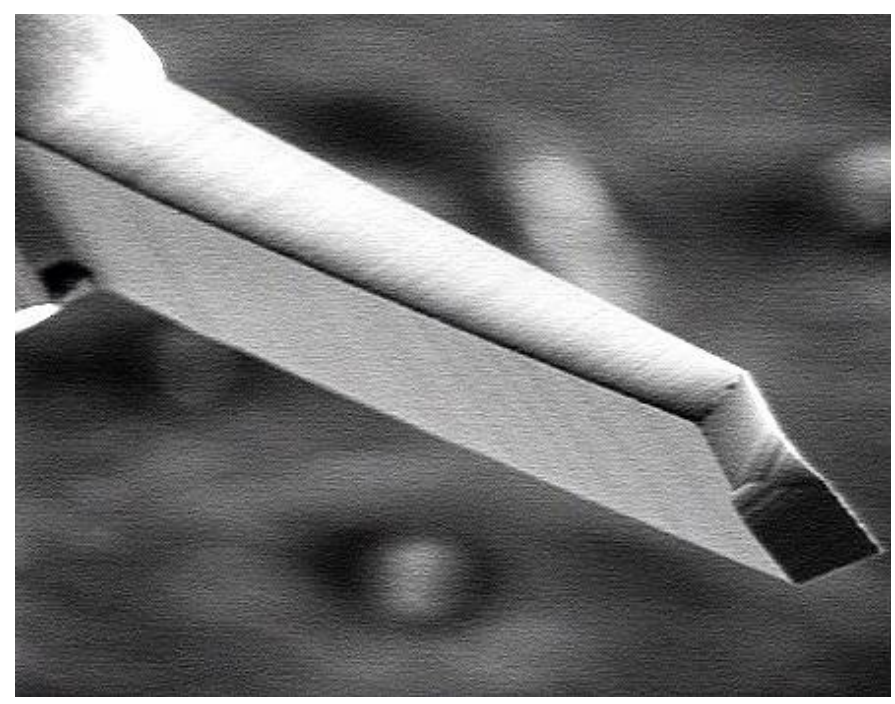

Figure 25. SEM image of 150 micrometer endmill 
Table 1. Optimized machining variables for machining Ti microtacks

\begin{tabular}{|c|c|}
\hline Spindle Speed & $8000 \mathrm{RPM}$ \\
\hline Feedrate & $50 \mu \mathrm{m} / \mathrm{s}$ \\
\hline Plunge Rate & $25 \mu \mathrm{m} / \mathrm{s}$ \\
\hline Depth of Cut & $12.50 \mu \mathrm{m}$ \\
\hline Cutting Fluid & Trico Misting Fluid \\
\hline
\end{tabular}

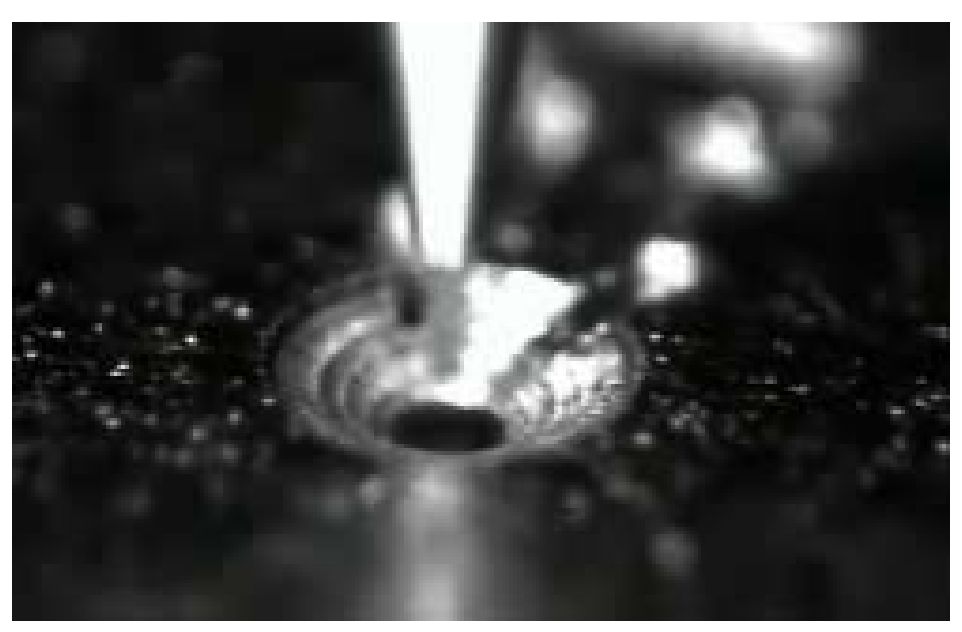

Figure 26. Image of machining process using 150 micrometer endmill in $\mathrm{Ti}$

Post-machining processing of the tacks consisted of removal of the two-part epoxy, as well as the burrs and surfaces flaws created during the micromilling process. The Ti coupon was placed in a pure Dichloromethane bath at room temperature for approximately two hours to dissolve the epoxy and release the microtacks from the silicon substrate. All burrs and flaws created during the machining process by the cutting tools (Figure 27) were etched away by placing the microtacks in a 40:1 dilution of $\mathrm{HF}$ at room temperature for 5 to 6 minutes. 


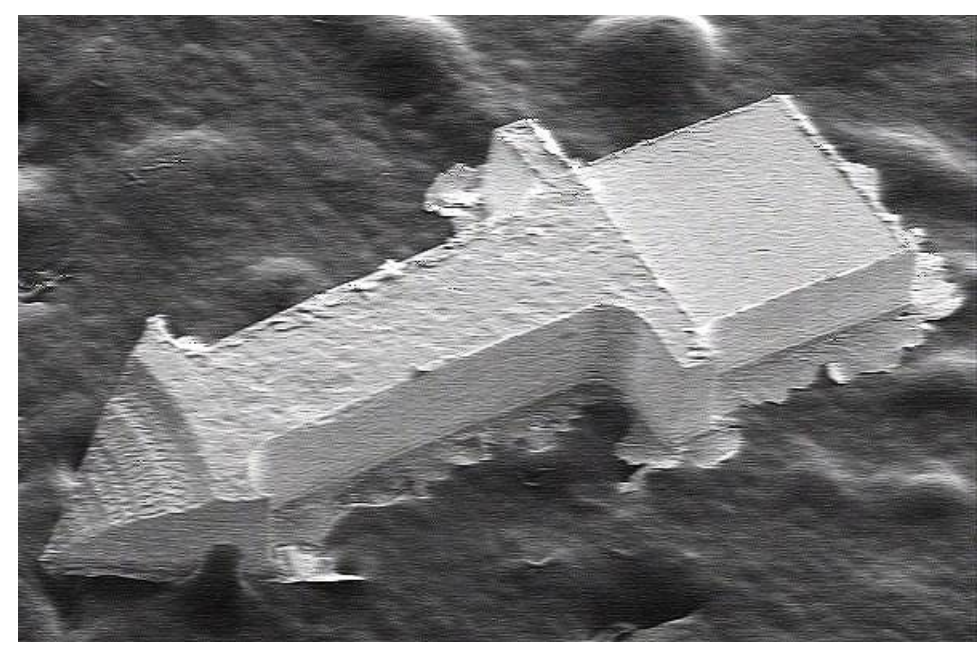

Figure 27. SEM image of Ti microtack with burrs and machining flaws

\subsection{Metrology}

Metrology was performed using the WYKO NT-2000. The microtacks were placed on a polished silicon wafer, to ensure flatness during the measurements. Standard operating procedures were used in vertical scanning interferometry (VSI) mode to measure the overall and critical dimensions of both the silicon and titanium microtacks. A two dimensional sketch of each microtack design is shown in Figure 28, all features and geometries that were measured are labeled accordingly and the original design specifications are listed with a brief description in Table 2. Three dimensional isometric images were recorded of each microtack using the SUPRA ${ }^{\mathrm{TM}}$ 35VP SEM as well. 

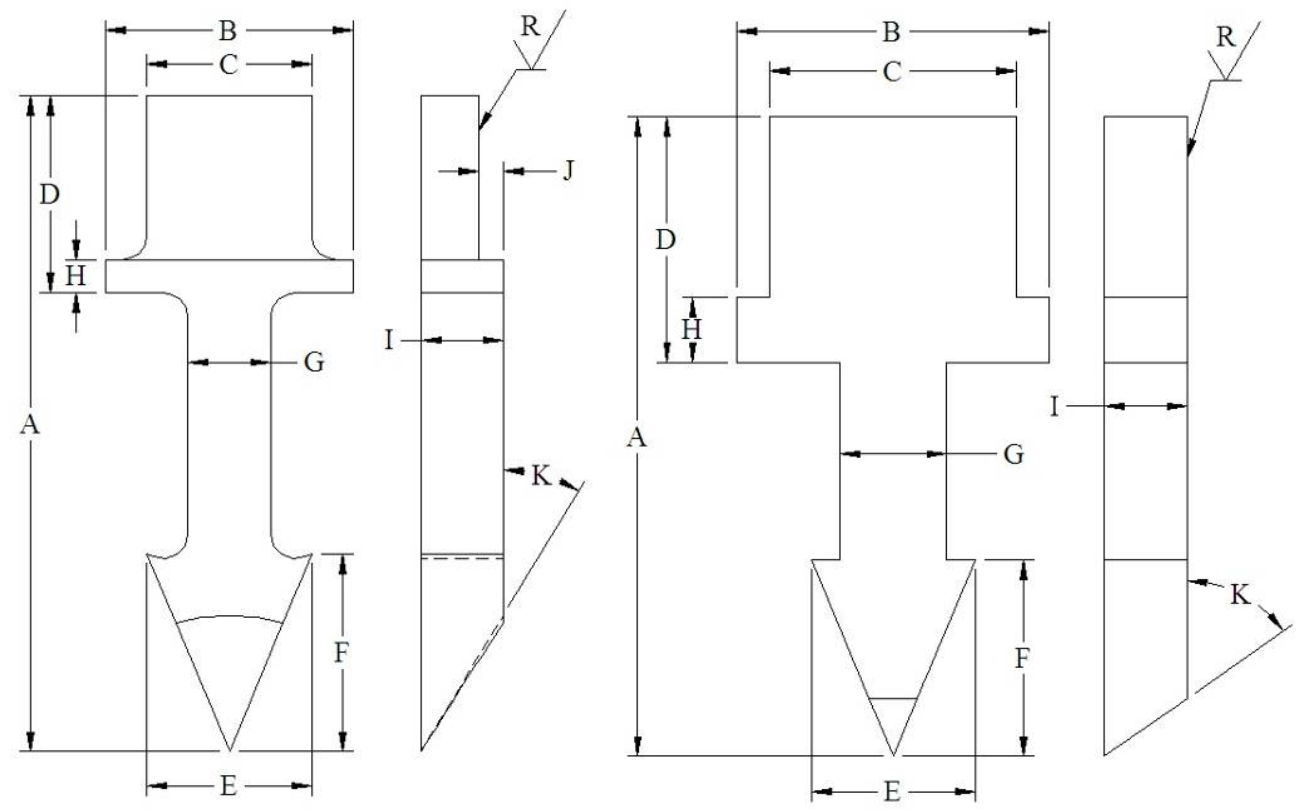

Figure 28. 2D sketch displaying the top and side views of the overall and critical dimensions measured for the microtack designs

Table 2. Designed dimensions for microtacks, in micrometers $(\mu \mathrm{m})$

\begin{tabular}{|c|c|c|c|}
\hline & Description & Titanium & Silicon \\
\hline A & Overall Length & 1950 & 1950 \\
\hline B & Overall Width & 750 & 950 \\
\hline C & Grip Head Width & 500 & 750 \\
\hline D & Grip Head Length & 500 & 750 \\
\hline E & Dual Barb Width & 600 & 500 \\
\hline F & Dual Barb Length & 500 & 600 \\
\hline G & Shaft Width & 250 & 325 \\
\hline H & Stop Thickness & 100 & 200 \\
\hline I & Overall Thickness & 250 & 250 \\
\hline J & Grip Step Depth & 100 & N.A. \\
\hline K & Tip Angle & $32^{\circ}$ & $54.74^{\circ}$ \\
\hline
\end{tabular}

\subsection{Determination of Buckling Force}

Buckling, as noted in prior sections, has been a source of failure for the silicon microtacks fabricated by Shire et al. (2002). With the microtack material and dimensions identified, buckling forces can be determined for each new microtack design using four 
different analytical methods. All four of these analytical methods assume that the tack geometry acts as a column with the same cross-sectional area as the microtacks and the microtacks have a clamped-free end condition [Juvinall 2000, Beer 1992] (Figures 29 and 30a).

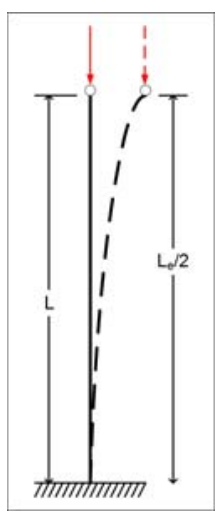

Figure 29. Clamped-Free end condition for buckling.

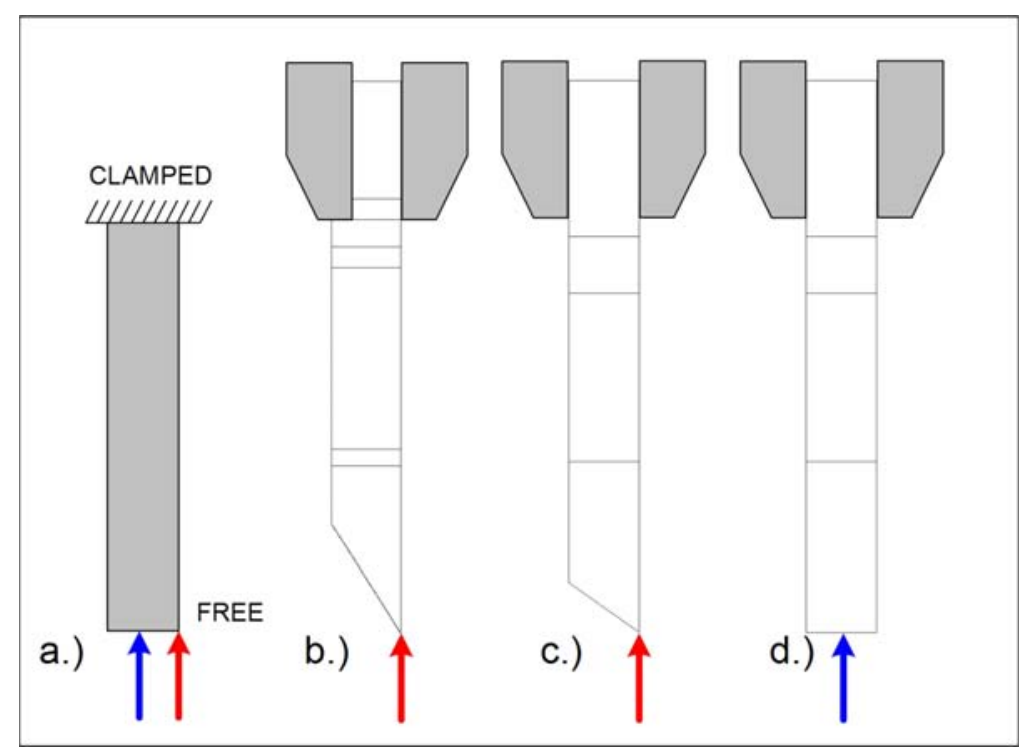

Figure 30. Comparison of microtacks for a: a) simply loaded column; b) titanium sharp tip; c) silicon pointed tip; and, d) silicon chisel tip

The first analytical method used to calculate the buckling force was based on the superposition method for a compressive and bending load combination. Specifically, Beer et al. (1992) states that 


$$
\sigma_{y}=\frac{P}{A}+\frac{M c}{I}
$$

Where $A$ is the cross-sectional area of the column, $I$ is the column moment of inertia, $c$ is the distance from the neutral axis to the outer most point of the column, $\sigma_{y}$ is the yield strength of the material, $P$ is the applied load, and $M$ is the moment that is applied to the column ( $P \cdot d, d$ is the distance from the centroid to point of application of the load). To determine the load at which the column would yield, solve for $P$ by

$$
P=\frac{\sigma_{y} A I}{A d c+I}
$$

For the titanium sharp tip and silicon pointed tip microtacks this equation is valid, but for the chisel tipped tack there would not be an induced moment due to load symmetry, therefore

$$
P=\sigma_{y} A
$$

A second method for computing the buckling force is Euler's equation:

$$
P_{c r}=\frac{\pi^{2} E I}{L_{e}^{2}}
$$

Where $E$ is the modulus of elasticity, $L_{e}$ is the effective column length where in the clamp-free end condition, $L_{e}=2.1 L$, and $P_{c r}$ is the critical load at which the column will buckle when a centric load is applied [Juvinall 2000]. With this method, assuming axial loading, the critical load will essentially be much higher than the actual case.

The third analytical method for the determining the buckling load incorporates the J. B. Johnson parabola equation for column buckling. The equation:

$$
P_{c r}=\sigma_{y}-\frac{\sigma_{y}^{2}}{4 \pi^{2} E}\left(\frac{L_{e}}{\rho}\right)^{2}
$$


where $\rho$ is the radius of gyration $(\rho=0.289 h)$ [Juvinall 2000], $\mathrm{h}$ is the thickness of the microtack ( $\mathrm{h}=250$ micrometers) and the quantity $\frac{L_{e}}{\rho}$ is the slenderness ratio which was 43.95. This signifies that the microtacks at their designated length of $1.500 \mathrm{~mm}$ are considered an intermediately long column [Juvinall 2000], which falls within the range for using the Johnson equation.

The fourth and final method for determining buckling force is the Secant Formula for eccentric column loading [Juvinall 2000, Beer 1992, Norton 1998]. This eccentricity occurs when the line of action of the applied load does not pass through the centroid of the column cross-section as seen in all tack designs. The equation for finding the critical eccentric load is:

$$
\sigma_{y}=\frac{P}{A}\left(1+\frac{e c}{\rho^{2}} \sec \left(\left(\frac{L}{2 \rho}\right) \sqrt{\frac{P}{E A}}\right)\right)
$$

where $e$ is the eccentric distance from the applied load to the centroidal axis. This equation is very similar to Equation (1) since it also accounts for both the applied compressive and bending loads, and combines them. The quantity $\frac{e c}{\rho^{2}}$ is called the eccentricity ratio, where it was equal to 2.99 for the titanium and silicon pointed tip microtack designs since the load is eccentrically applied while the eccentricity ratio is equal to 0.5 for the silicon chisel tip tack. The smaller ratio for the chisel tipped microtack would account for the typical variations in the loading eccentricity for a concentrically loaded structure [Norton 1998].

Table 3 lists all the critical loads required to buckle the simply clamped-free column for each of the analytical methods. The Euler method computed the highest load 
primarily due to the equation assuming a centric load. The other three methods computed more realistic loads, all under 1000 grams $_{\text {force, }}$, with the Secant and Bending methods yielding similar results for each design. The Johnson equation is also valid since the microtack 'columns' possess slenderness ratios within its calculated range. It is anticipated that the measured buckling failure loads for the microtacks will fall between the values computed by the Johnson Equation (Eqn. 4) for the maximum load and the Secant/Bending Equations (Eqns. 1,2 and 5) for the minimum load. The calculations for each microtack can be seen in Appendix II.

Table 3. Different theoretical values for buckling force for each microtack design using the four analytical methods

\begin{tabular}{|c|c|c|c|c|}
\hline & Bending & Euler & Johnson & Secant \\
\hline Titanium & 222.99 & 3828.65 & 840.12 & 211.90 \\
\hline Silicon Pointed Tip & 248.47 & 4827.08 & 988.78 & 237.53 \\
\hline Silicon Chisel Tip & 993.88 & 4827.08 & 988.78 & 624.40 \\
\hline \multicolumn{5}{|c}{ Units $=$ grams $_{\text {force }}{ }^{* *}$} \\
\hline
\end{tabular}

\subsection{Characterization of Microtack Performance in Fiber Reinforced Silicone Rubber Gasket Material}

Prior to testing the microtacks in biological eye tissue, the tacks were characterized in a 380 micron thick sample $(25 \mathrm{~mm}$ x $125 \mathrm{~mm})$ of biomedical grade fiber reinforced silicone rubber gasket material (Specialty Manufacturing, Inc., Saginaw, MI) with a Shore durometer hardness of 40 . This was the material of choice due to its availability in our laboratory and its ease of use for the comparative tests between microtack designs.

Each silicone gasket sample was cut to size, special care was given to maintain similar orientation of the reinforcement fibers, so repeatable and comparable results could 
be obtained between tests (Figure 29). Three holes were punched into each silicone gasket sample, to match those in the vacuum fixture. The test sample was then placed, under tension $(\sim 1737 \pm 30.5$ grams $[$ Appendix II] $)$, over the vacuum fixture via three tightened screws (Figure 30) to reduce the overall compliance of the material [Frick 2001] during testing. Thereby, reducing the amount displacement required to overcome the residual compliance of the reinforced material. The insertion and retention force experiments were conducted in the RSA III system described in Chapter 3. The operating parameters and testing procedures implemented in the silicon gasket material experiments are described below in section 4.7.

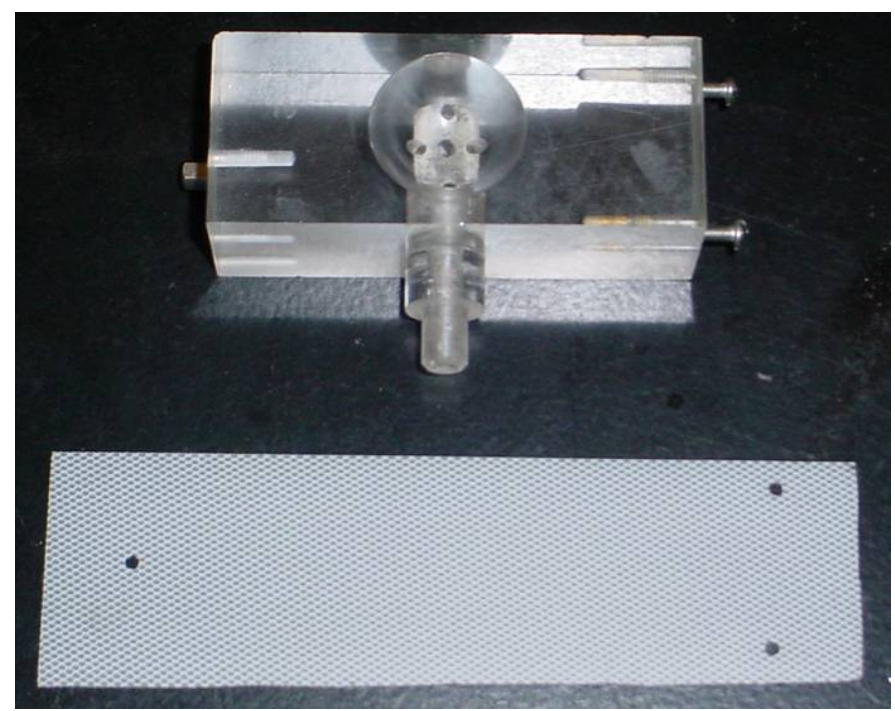

Figure 31. Prepared gasket material alongside vacuum fixture 


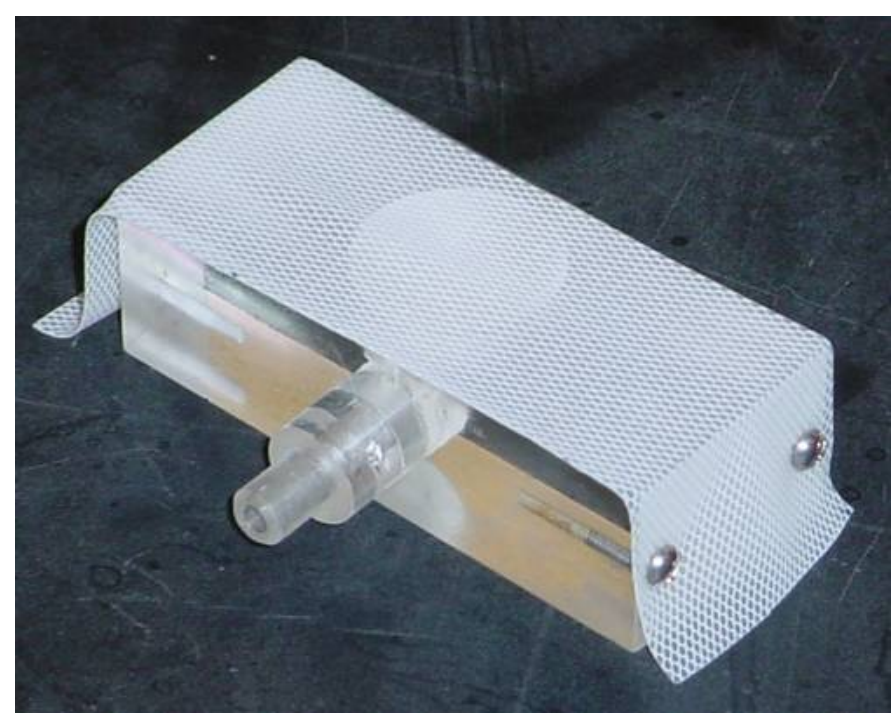

Figure 32. Gasket material mounted under tension over the vacuum fixture

\subsection{Characterization of the Microtack Performance in a Porcine Eye}

After characterization of the microtacks in the silicon gasket material, the microtacks were tested in biological tissue, i.e. porcine eyes. The porcine eyes were donated by Swift, Inc. in Louisville, KY. Since the eyes were not in suitable condition for immediate experimentation due to an abundance of conjunctiva and residual ocular muscle tissue, surgical scissors and forceps were used to remove the remaining tissue, leaving the scleral tissue, cornea, and clipped optic nerve exposed for testing. Subsequently, the eyes were preserved in a Saline solution ( $0.9 \%$ sodium concentration) and stored in refrigeration for no more than 3 days. After proper preparation of the eyes, the eyes were mounted in the vacuum fixture with the optic nerve protruding out to top of the fixture. Saline was applied every 5 to 10 minutes to the secured eye specimen via pipette to prevent the ocular tissue from drying. The insertion and retention force experiments were conducted in the RSA III system described in Chapter 3. The 
operating parameters and testing procedures implemented in the biological tissue experiments are described below in section 4.7.

\subsection{Insertion and Retention Force Experiments}

The force measurement experiments were performed using the RSA III rheology system described in Chapter 3. Prior to performing the insertion and retention tests, the high and low load transducers were calibrated to ensure the validity of the results. The calibration process consisted of hanging known masses from the high and low load transducers and adjusting the output reading to match the known mass (TA Instruments 2003). The complete calibration process is fully described in Appendix II.

A testing protocol was developed to ensure each tack design would be investigated in the same manner. The force measurement experiments were partitioned into three zones: insertion, hold, and removal, based upon the direction of microtack motion [Bzostek 1999]. During the insertion zone, the motion of each tack was inserted into the substrate and the compressive force encountered by the microtack increased until it punctured through the test sample. The Hold zone occurred when the tack was held in a static position for 1 second, allowing the test specimen to settle prior to removal. During the removal zone, the compressive force quickly reduces to zero and the removal (tensile) force created by the tack increases until the tack is entirely removed from the test specimen, at which time the force level again returns to zero. A constant microtack velocity of $1 \mathrm{~mm} / \mathrm{s}$ was used for both the insertion and removal zones. The input displacements incremented until insertion and removal occurred. This process was performed on each tack design and test sample. 
Initially, the microtacks were inserted into the synthetic rubber samples. The vacuum fixture with mounted material sample was placed in the RSA III system. Using tweezers, along with a steady hand under a lighted stereo microscope, the microtack was securely positioned in the micro-clamp and tightened. The micro-clamp was then mounted into the RSA3 (Figure 31).

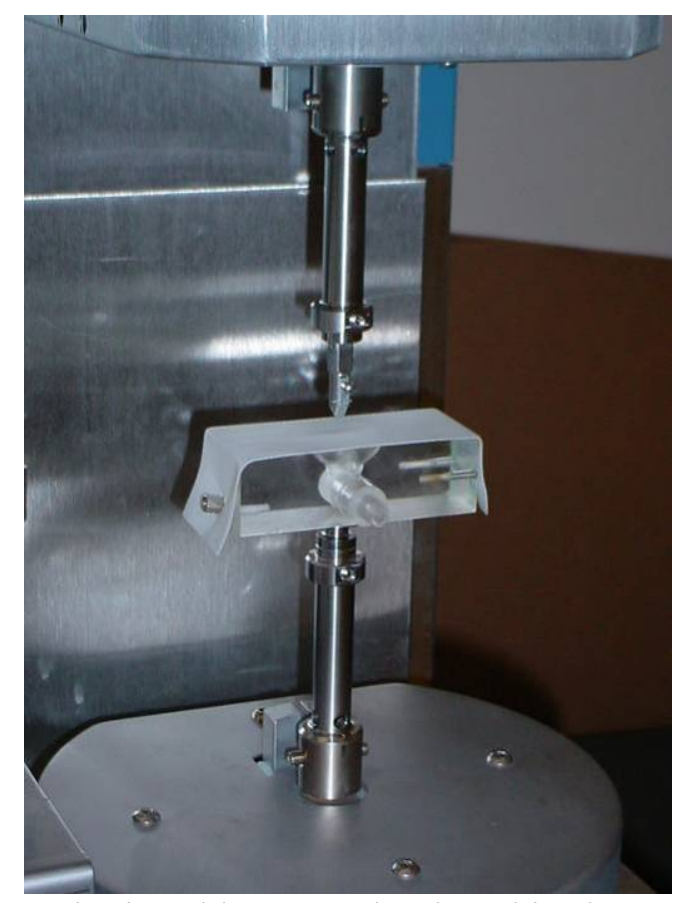

Figure 33. Synthetic rubber sample placed in the RSA III system

A stereo microscope with a mounted CCD camera plugged into a viewing monitor was used to observe the microtack during testing. The RSA III Z-axis traversed down until the tip of the microtack just touched (i.e. visual check and force detection on RSA III) the silicone gasket material. To determine the amount of displacement needed to overcome the residual compliance in the sample, the user simply started at the low end of the scale, $\sim 500$ micrometers of displacement, and increased in 500 micrometer increments until tack penetration occurred. There were 10 force measurement experiments performed in each silicone gasket sample, similar to the one series of images in Figure 34. One 
silicone gasket sample was used per microtack, however, the silicone gasket sample holding fixture was moved $1 \mathrm{~mm}$ to ensure that the microtacks were not inserted in the same location more than once.

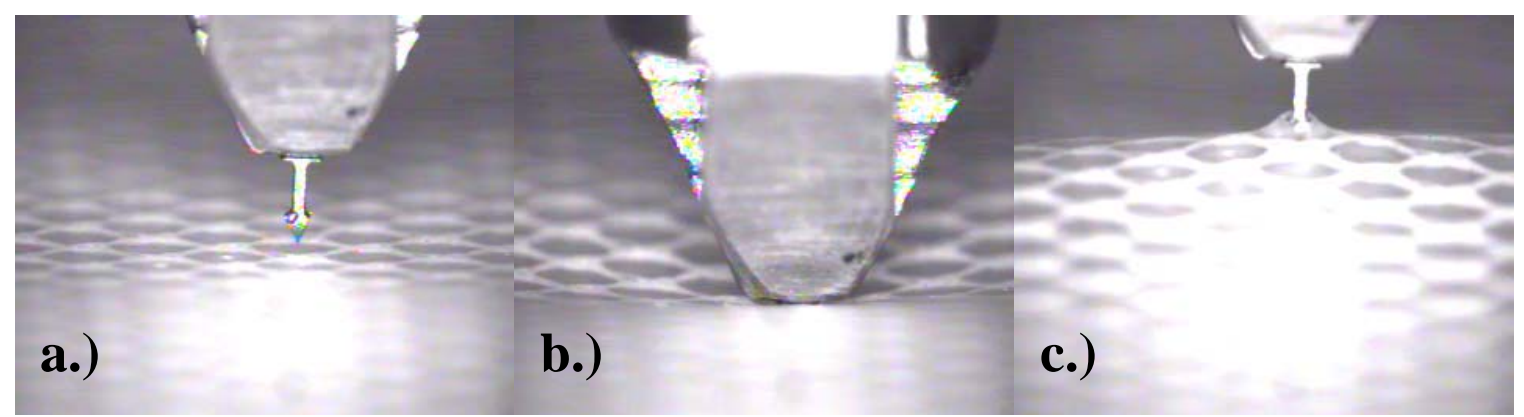

Figure 34. Series of images from force measurement experiment, a.) Touch, b.) Insertion, c.) Removal

The force measurement experiments with the porcine samples consisted of the same protocol as described for the silicone gasket material, except for the requisite vacuum line connected to the vacuum fixture (Figure $35 \& 36$ ). Tissue puncture tests were performed primarily in the region surrounding the optic nerve since this area is where the implant would typically be placed inside the eye (Figure 37). Compliance of the biological tissue was also a major issue and had to be overcome (Figure 38). The same procedure was used to determine input displacement as well. 


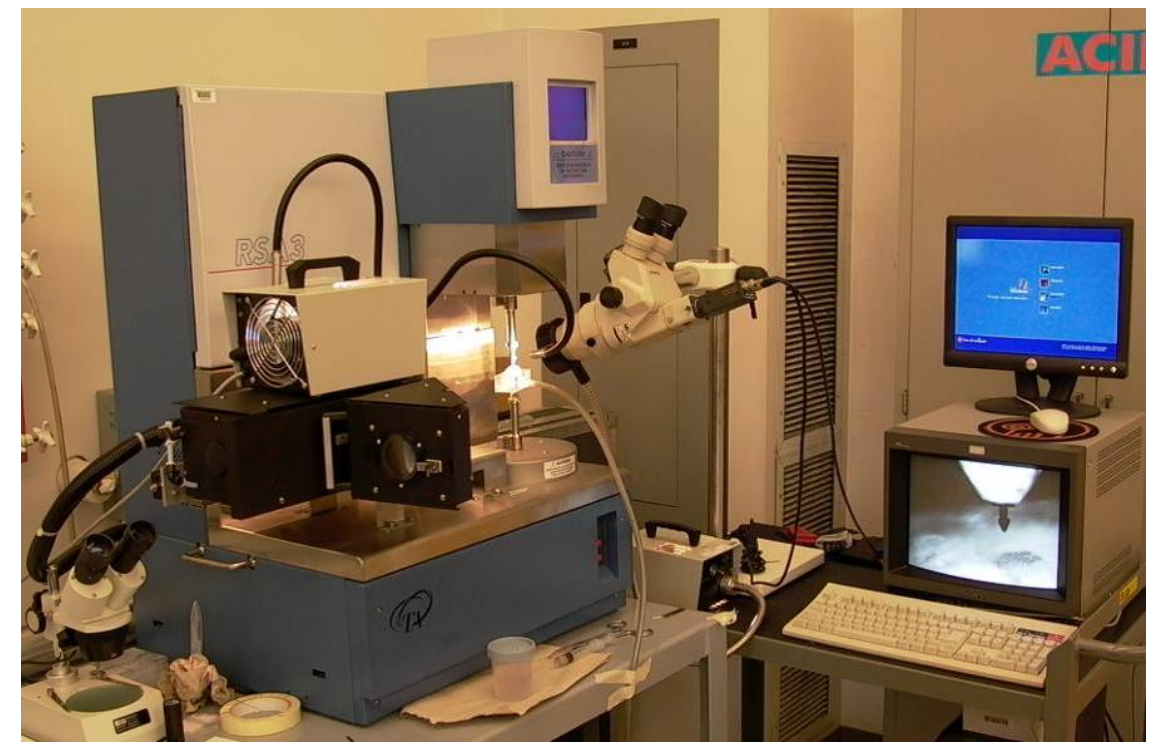

Figure 35. Overall test setup for insertion and retention force experiments in the eyes

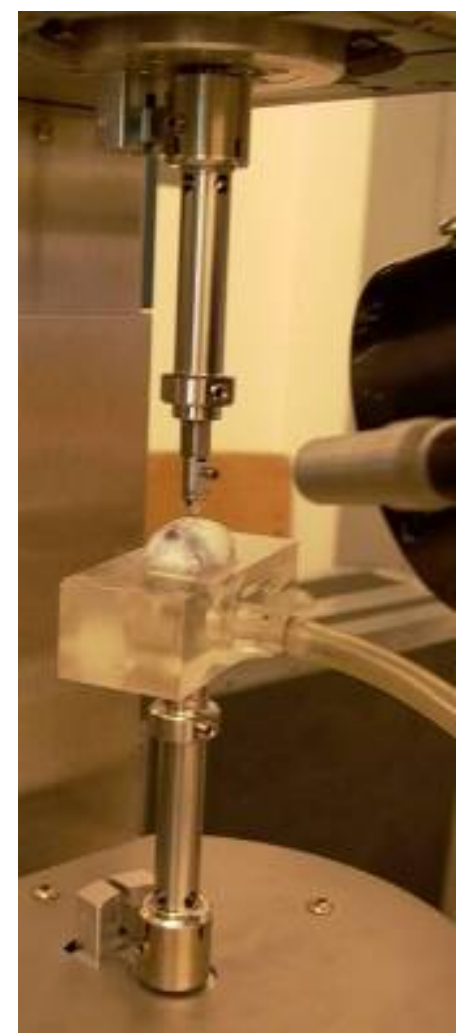

Figure 36. Close up of vacuum fixture holding a porcine eye 


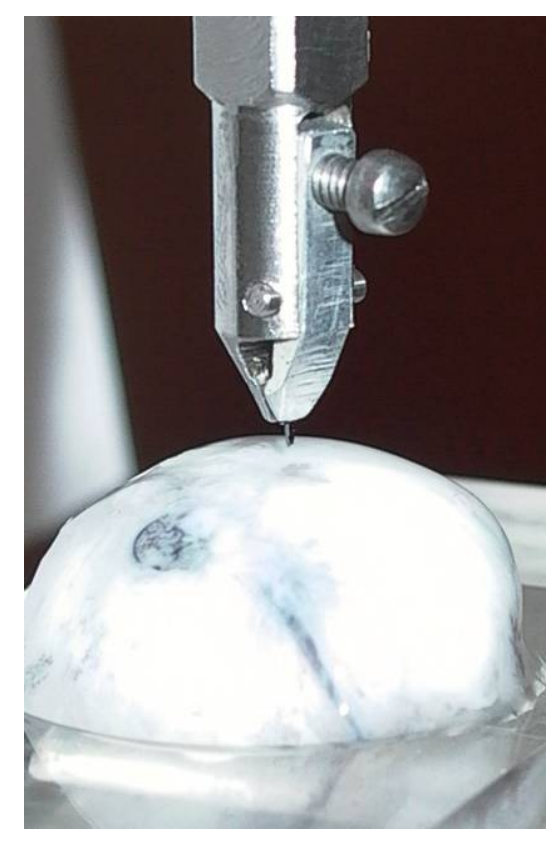

Figure 37. Typical placement of tack for insertion

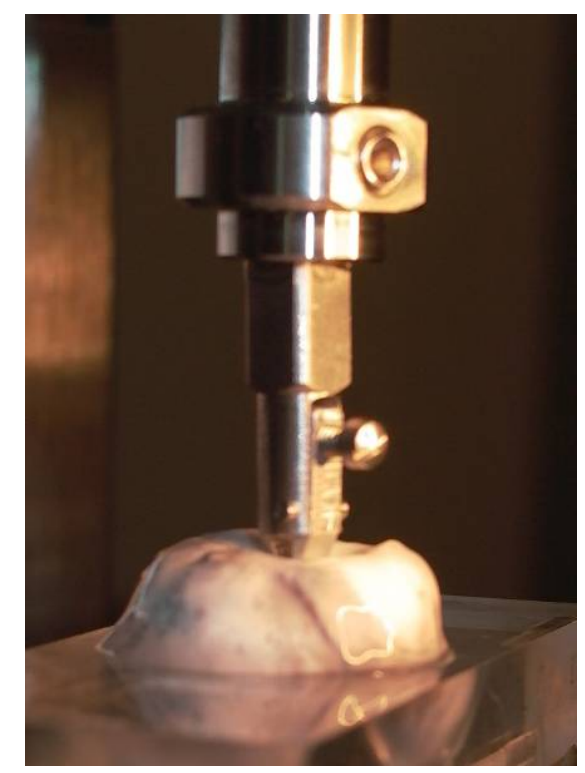

Figure 38. Insertion of microtack demonstrating tissue compliance issues

\subsection{Data Extraction and Analysis}

The three different microtack designs were tested in two different sample materials: fiber reinforced synthetic rubber gasket material and porcine eye tissue. Ten 
insertion/removal tests were performed on each microtack unless the tack was damaged due to breakage or bending under high insertion. The data in the form of an ASCII text file was retrieved from each force measurement experiment consisted of the time duration (seconds), displacement (millimeters), and normal force (grams). Combinations of the recorded data was analyzed and input into the plots listed:

- Normal Force versus Time

- Normal Force versus Displacement

- Total Force - Derived from the Normal Force curve

The data files were exported as text files (.txt) from the RSA III and imported into a Microsoft Excel worksheet. The data was recorded at a sample rate of 300 sample points per zone, the maximum number available with the RSA III system. This sample rate exceeds the minimum number required by the Nyquist Criterion to avoid data aliasing [Dally 1993, Taylor 1997]. The duration for each of the three zones previously described are: 1) insertion: $\Delta \mathrm{t}=3$ seconds, hold: $\Delta \mathrm{t}=1$ second, and removal: $\Delta \mathrm{t}=6$ seconds. Table 4 describes the sample rates for each zone.

Table 4. Sample rates for each zone of experimental setup

\begin{tabular}{|c|c|c|}
\hline & $\Delta \mathrm{t}(\mathrm{s})$ & Sample Rate $(\mathrm{Hz})$ \\
\hline Insertion & 3.00 & 100 \\
\hline Hold & 1.00 & 300 \\
\hline Removal & 6.00 & 50 \\
\hline
\end{tabular}

Total force is an alternative quantitative value that can be used for comparing force data between microtack designs. The total force was computed as the area under the curve for the normal force versus time plot and was calculated using MathCAD 2001 Professional (Mathsoft Inc.). The data file was imported into the developed software 
macro (Appendix II) and converted to a single line using the lspline() command. The area under the curve was calculated by integrating the resulting line equation. There were two areas of interest, the area during insertion and the area during removal. Each of these areas was integrated separately. The area of insertion was integrated from $t=0$ seconds ( $F=0$ grams) to the time of maximum insertion force occurred. The area of removal was integrated from the time removal began $t \sim 4$ seconds $(F=0$ grams) to the time of maximum removal force occurred. A student's paired t-Test, with a two-tailed distribution was performed on all results determining significance. 


\section{RESULTS AND DISCUSSION}

\subsection{Microtack Fabrication}

\subsubsection{Fabrication of Chisel Tip and Pointed Tip Silicon Microtacks}

A total of 96 silicon microtacks of each design were batch fabricated using the DRIE and anisotropic etch processes (Figures 39 and 40). The DRIE process was able to produce a sharp edge in the silicon; however, the overall shape is a chiseled tip similar to the microtacks produced by Shire, et al. (2002). SEM images of all other microfabricated silicon microtacks are included in Appendix III. Four silicon microtacks were measured using the WYKO. The top view and 3D rendering of a single Si microtack is shown in Figure 41a) and 41b). The recorded measurements are shown in Table 6.

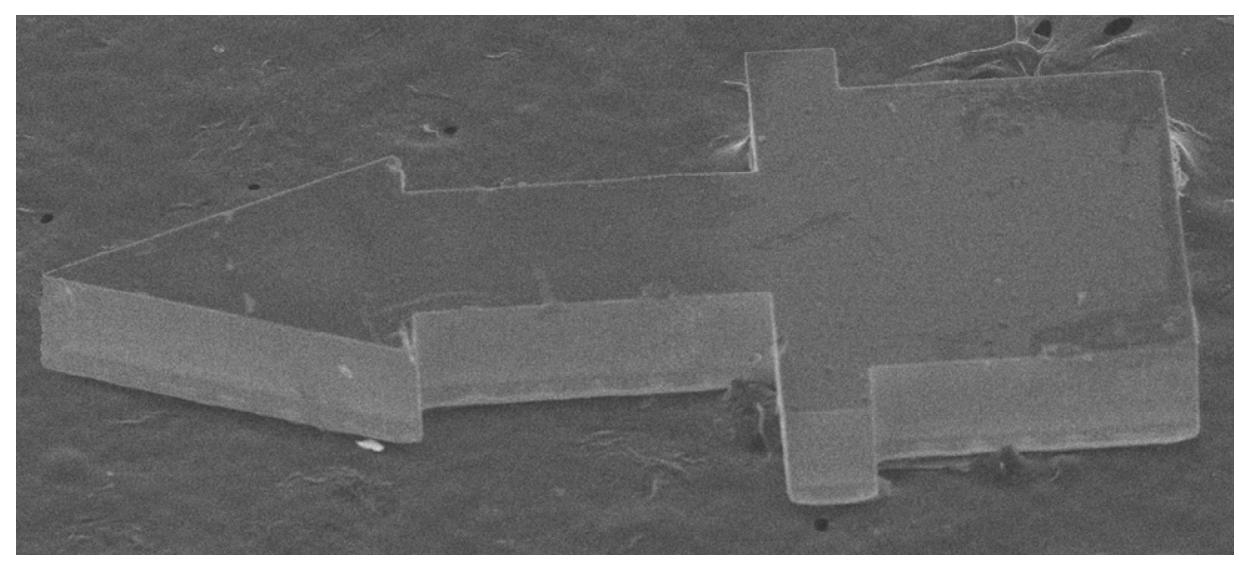

Figure 39. SEM images of silicon chisel tip microtack 


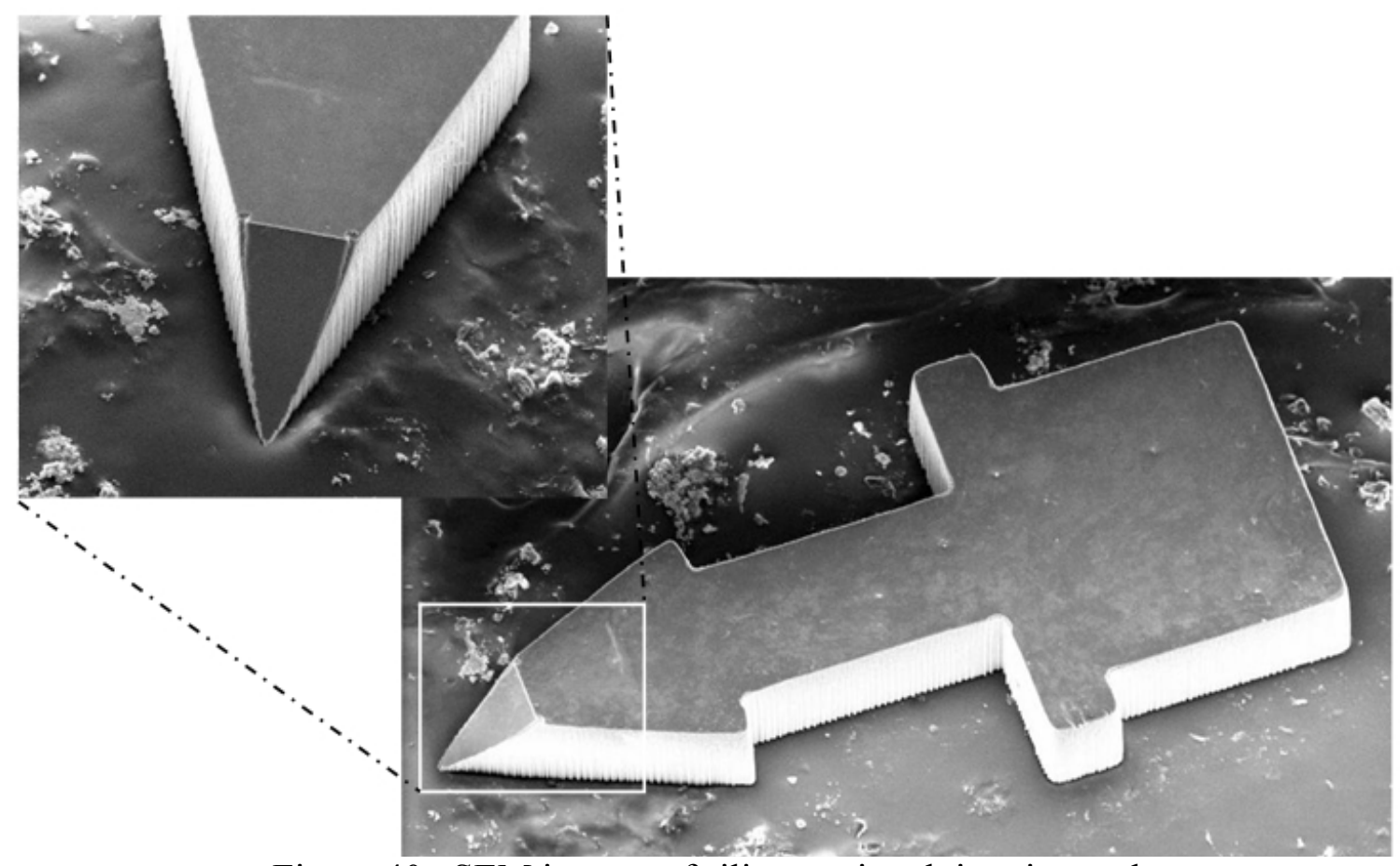

Figure 40. SEM images of silicon pointed tip microtack
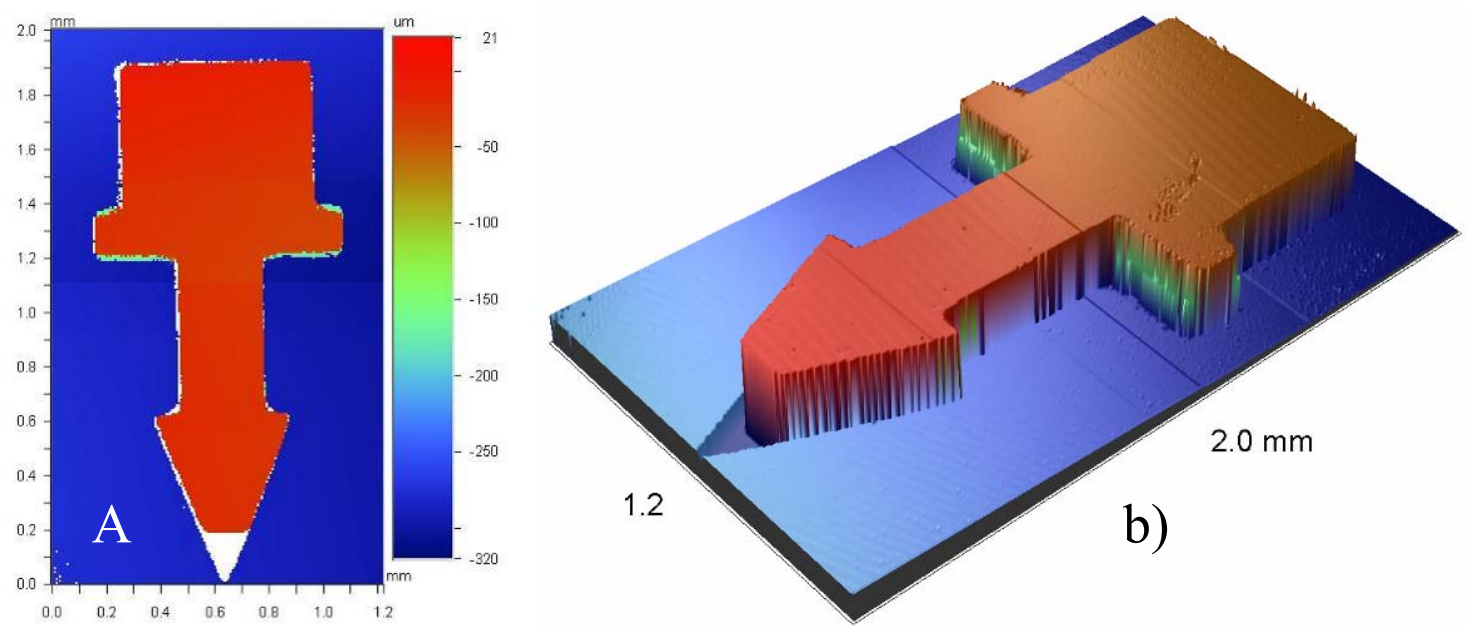

Figure 41. a) Top view and b) 3D rendering of a silicon pointed tip microtack scanned using the WYKO 
Table 5. List of designed, actual and percent difference of silicon pointed tip microtacks

\begin{tabular}{|c|c|c|c|c|}
\cline { 2 - 4 } \multicolumn{1}{c|}{} & \multicolumn{5}{c|}{ Silicon Chisel/Pointed Tip Microtack } \\
\cline { 2 - 5 } \multicolumn{1}{c|}{ Design } & Actual \pm SD & $\%$ DIFF \\
\hline A & 1950 & $1918 \pm 11.84$ & $-1.7 \%$ \\
\hline B & 950 & $941.5 \pm 13.99$ & $-0.9 \%$ \\
\hline C & 750 & $738.5 \pm 7.51$ & $-1.5 \%$ \\
\hline D & 750 & $746.8 \pm 8.50$ & $-0.4 \%$ \\
\hline E & 500 & $506.5 \pm 4.04$ & $1.3 \%$ \\
\hline F & 600 & $586.5 \pm 14.06$ & $-2.3 \%$ \\
\hline G & 325 & $327.3 \pm 6.70$ & $0.7 \%$ \\
\hline H & 200 & $199.0 \pm 4.62$ & $-0.5 \%$ \\
\hline I & 250 & $267.8 \pm 4.50$ & $7.1 \%$ \\
\hline J & N.A. & N.A. & N.A. \\
\hline K & 54.74 & $54.0 \pm .430$ & $-1.3 \%$ \\
\hline
\end{tabular}

The dimensions measured for the chiseled and pointed silicon microtacks vary from the design parameters; there are several potential reasons for these discrepancies. Specifically, in the case of the undersized dimensions, the photolithographic process could have contributed to this discrepancy since overexposure of the photoresist would cause the etched region to be larger. In addition, under cutting of the masked region during the DRIE process could also contribute to excess material being removed.

The surface roughness ( $\mathrm{R}$ in figure in Table 6) of the silicon microtack was also measured using the WYKO. The root-mean-squared roughness $\left(\mathrm{R}_{\mathrm{q}}\right)$ was $0.236 \pm 0.040$ $\mu \mathrm{m}$. The surface roughness of the silicon microtack was relatively low due to the wafer being double-sided polished, minimizing surface roughness.

\subsubsection{Fabrication of 3-D Sharp Tip Titanium Microtacks}

A total of 10 titanium microtacks were successfully machined using the ultrahigh-precision micromilling process (Figure 42), which clearly illustrates the 3-D 
features that the micromilling technique is capable of producing in the new microtack design. SEM images of all other machined titanium microtacks are included in Appendix III.

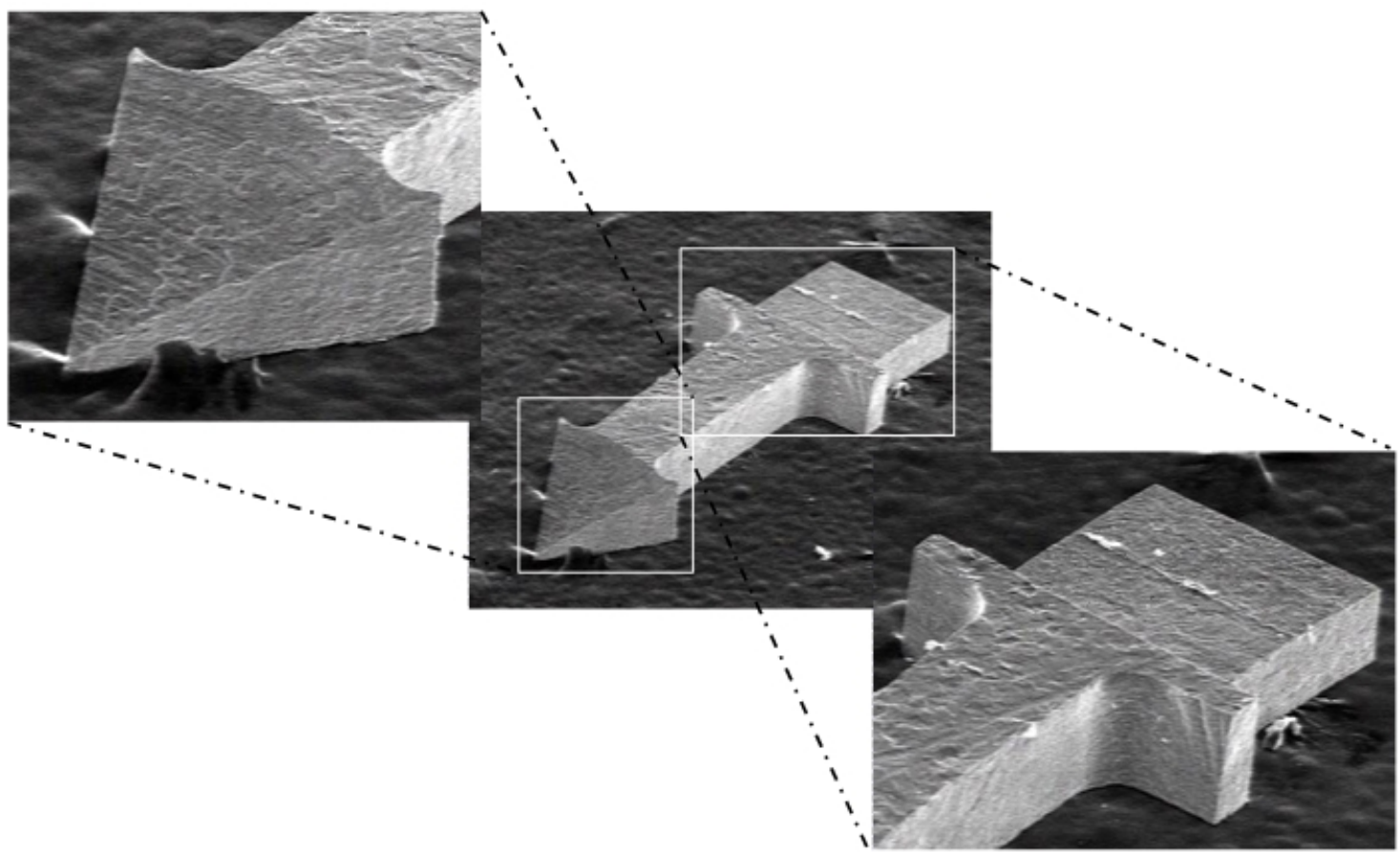

Figure 42. SEM images of machined and deburred titanium microtack

Metrology was performed using the WYKO VSI to measure the overall and critical dimensions of four titanium microtacks. Vertical scanning interferometer output images were obtained for each microtack (Figure 43) and the dimensions defined previously were measured. The recorded metrology data showed that all of the measured dimensions of the titanium tacks were within $6.5 \%$ of the design parameters (Table 6). 

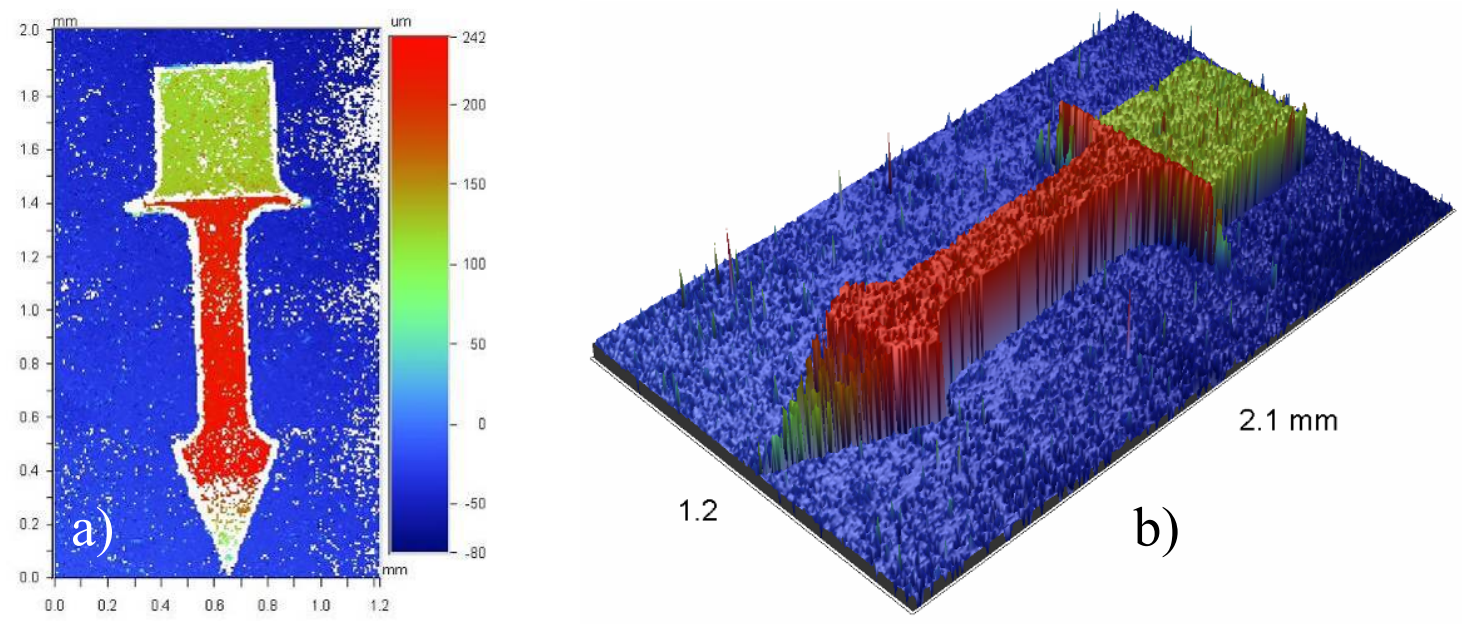

Figure 43. Typical Vertical Scanning Interferometer output image for a Titanium microtack: a) Top view; and, b) 3D rendering

Table 6. WYKO metrology data for the measured titanium microtacks

\begin{tabular}{|c|c|c|c|c|}
\cline { 2 - 4 } \multicolumn{1}{c|}{} & \multicolumn{5}{c|}{ Titanium Tapered Tip Microtack } \\
\cline { 2 - 5 } \multicolumn{1}{c|}{ Design } & Actual \pm SD & $\%$ DIFF \\
\hline A & 1950 & $1920.5 \pm 4.57$ & $-1.5 \%$ \\
\hline B & 750 & $709.3 \pm 18.98$ & $-5.4 \%$ \\
\hline C & 500 & $471.0 \pm 6.90$ & $-5.8 \%$ \\
\hline D & 600 & $573.2 \pm 2.52$ & $-4.5 \%$ \\
\hline E & 450 & $424.5 \pm 4.80$ & $-5.7 \%$ \\
\hline F & 550 & $514 \pm 10.24$ & $-6.5 \%$ \\
\hline G & 225 & $222.9 \pm 10.12$ & $-1.0 \%$ \\
\hline H & 100 & $98.7 \pm 2.04$ & $-1.3 \%$ \\
\hline I & 250 & $258 \pm 4.35$ & $3.2 \%$ \\
\hline J & 90 & $87.6 \pm 8.90$ & $-2.7 \%$ \\
\hline K & 32 & $32.7 \pm .86$ & $2.1 \%$ \\
\hline
\end{tabular}

All of the dimensions measured (A through $\mathrm{J}$ in Table 6) are less than the design parameters; there are several reasons for this phenomenon. When machining pure titanium, if the machining parameters aren't optimized, the chips removed during the machining process tend to gall onto the cutting tool due to the ductility of the material. This can increase the wear on the endmill; thereby, elevating the cutting forces required 
to machine the material, which result in an increase in the amount of deflection of the endmill. Consequently, this increase in endmill deflection can lead to machining an oversized hole or slot [Shaw 2005]. Additionally, when an endmill is placed into the micromilling machine collet system it tends to have a small amount of eccentricity. The collet system used on the micromilling system (ER 16) has an eccentricity tolerance of $\sim 10$ micrometers. If this issue is not compensated for, it will cause discrepancies in the final product dimensions due to the increased movement of the tool during the machining process. The chemical deburring process will also cause the titanium microtacks to be undersized because the HF etching procedure also results in the loss of material since it is a chemical material removal process, although the material loss by this process will be minute compared to the issues previously stated. However, the combination of all three of these factors will contribute to the error in the final titanium microtack dimensions.

The surface roughness ( $\mathrm{R}$ in figure in Table 6) of the titanium microtack was also measured using the WYKO. The root-mean-squared roughness $\left(\mathrm{R}_{\mathrm{q}}\right)$ was $7.33 \pm 1.26 \mu \mathrm{m}$. The surface roughness of the titanium microtack was relatively high due to the chemical deburring process. The etching process produced rough pitted areas throughout the surface area of the microtack, increasing the surface roughness. This surface roughness could possibly promote tissue in growth which may assist in securing the microtack and implant in place. 


\subsection{Force Determination Experiments}

\subsubsection{Characterization of Microtack Performance in a Fiber Reinforced Silicone Rubber Material}

All microtack designs were tested in a fiber reinforced silicone rubber material using the protocols defined in Chapter 4. Specifically, each tack design was inserted into the material and normal force versus time plots were obtained (Figure 44) and the three zones previously described are clearly distinguished. The first peak in the negative force domain represents the insertion (Zone 1) of the microtack into the synthetic rubber material. The horizontal line in the hold zone (Zone 2) corresponds to the constant force applied once the synthetic rubber settles on the grip end of the microtack during the one second motionless hold period. The second peak in the positive force domain represents the retention force (Zone 3) required to completely remove the microtack from the specimen. Once the tack is fully removed, the force returned to zero. The plot also shows the differences in load between the microtack designs. As demonstrated in Figure 44, the titanium microtack requires less force for insertion and greater force for removal, while the silicon pointed tip tack requires greater insertion force than the titanium and less than silicon chisel tip. The removal force is approximately the same for the two silicon designs; this is primarily due to the fact that the 'barbs' on both silicon tack designs possess essentially the same dimensions and geometry. Note the additional spike at the end of the insertion peaks for the two silicon microtacks compared to the titanium microtack which does not have this additional spike. This spike is due to the tip geometry of the titanium microtack which induces a smoother transition during insertion, while the silicon tip designs "pop" through the interface resulting in the additional peak. 
Furthermore, the amplitude of this additional peak is smaller for the pointed tip silicon microtack compared to the chisel tip design, which further corroborates this rationale.

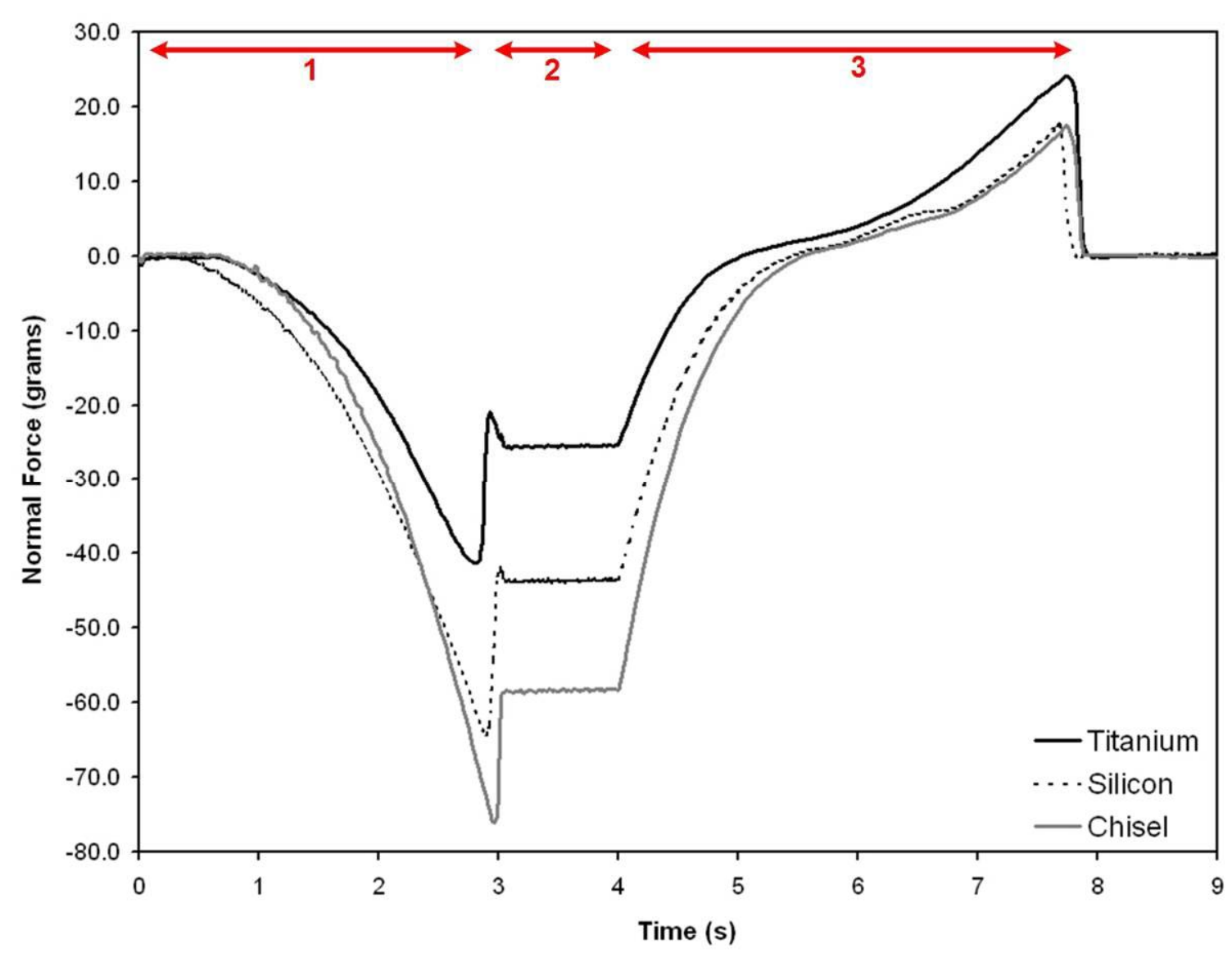

Figure 44. Typical normal force versus time plot for all microtacks tested in the fiber reinforced silicone rubber gasket material

Figure 45 illustrates the average insertion and removal forces, respectively, for all the experiments for each microtack design. The average maximum insertion force of the titanium microtack $(42.90 \pm 5.20$ grams $)$ was found to be significantly $\left(\alpha_{\text {insertion }}<<0.001\right)$ smaller than the insertion force for the silicon chisel tip (41.7\% higher) and silicon pointed tip (35.4\% higher) microtacks. The average maximum removal force of the titanium microtack $(23.54 \pm 1.50$ grams $)$ was found to be significantly $\left(\alpha_{\text {removal }}<<0.001\right)$ 
higher than the silicon chisel tip (28.1\% lower) and silicon pointed tip (30.1\% lower) microtacks. In comparing the results between the two silicon tip designs, the pointed tip was found to require significantly $\left(\alpha_{\text {insertion }}<<0.001\right)$ less insertion force than the chisel tip design. Similarly, the retention forces were significantly $\left(\alpha_{\text {removal }}<<0.001\right)$ higher for the silicon pointed tip design compared to the chisel tip design. These results indicate that all of the hypotheses are accepted for the fiber reinforced silicone rubber material. Individual microtack results are included in Appendix IV.

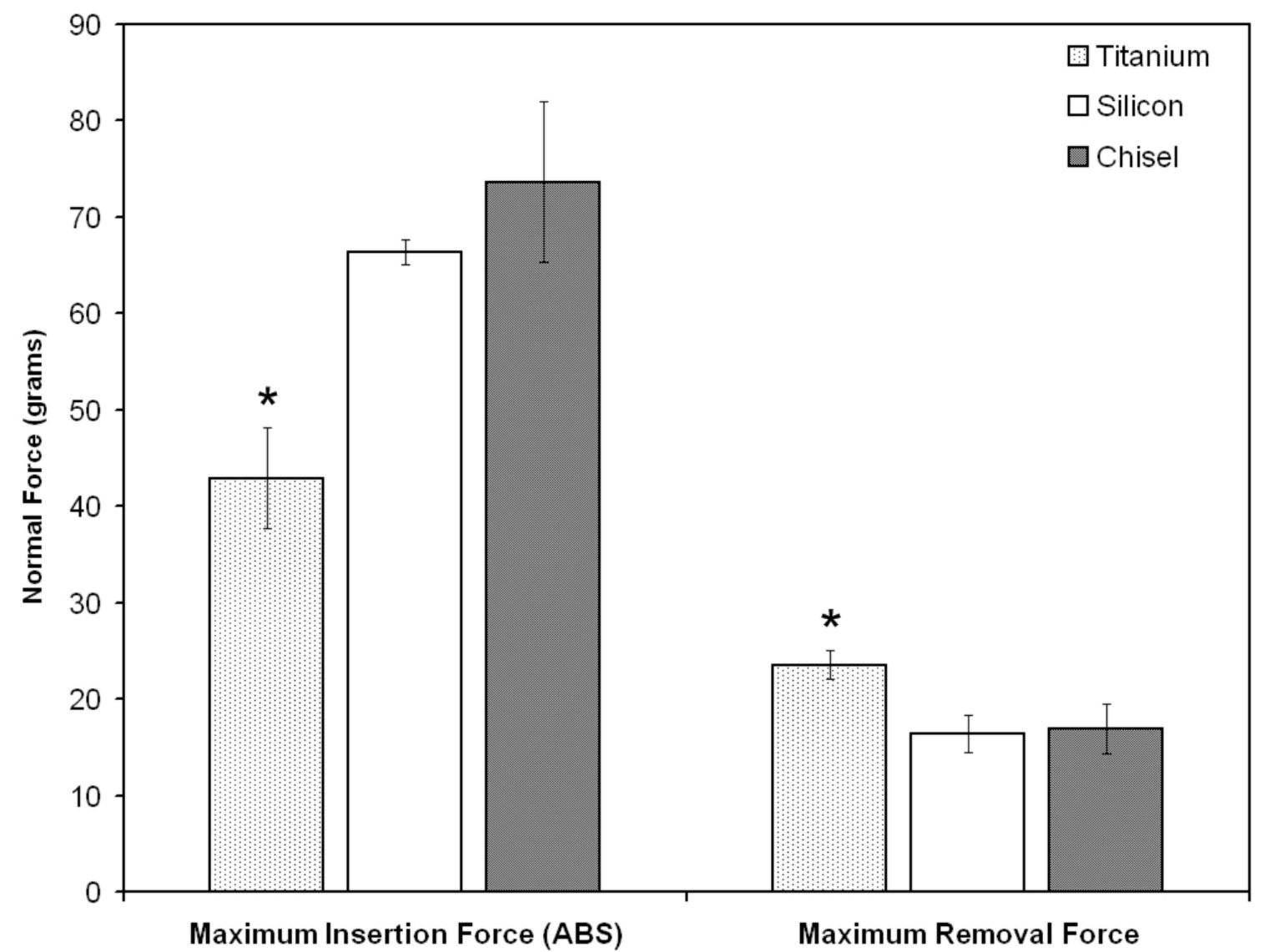

Figure 45. Average maximum insertion and removal forces for all microtack designs in the fiber reinforced silicone rubber gasket material. $\left({ }^{*} \alpha<<0.001\right)$ 
Figure 46 displays a typical normal force versus displacement plot for a single run using each type of microtack design. Three zones are observed. Zone 1 (Insertion) starts at the plot origin represented in red and extends to the peak in the negative force domain. This point signifies the complete insertion of the microtack into the synthetic rubber material. The vertical line in the positive displacement domain represents Zone 2 (Hold) displayed in green. Zone 3 (Removal) displayed in blue immediately follows the Hold. The peak in the positive force domain represents the total retention force required to completely remove the microtack from the specimen. Once the tack is fully removed, the force returns to zero. The plot also shows the differences in the force and the required displacement between the microtack designs.

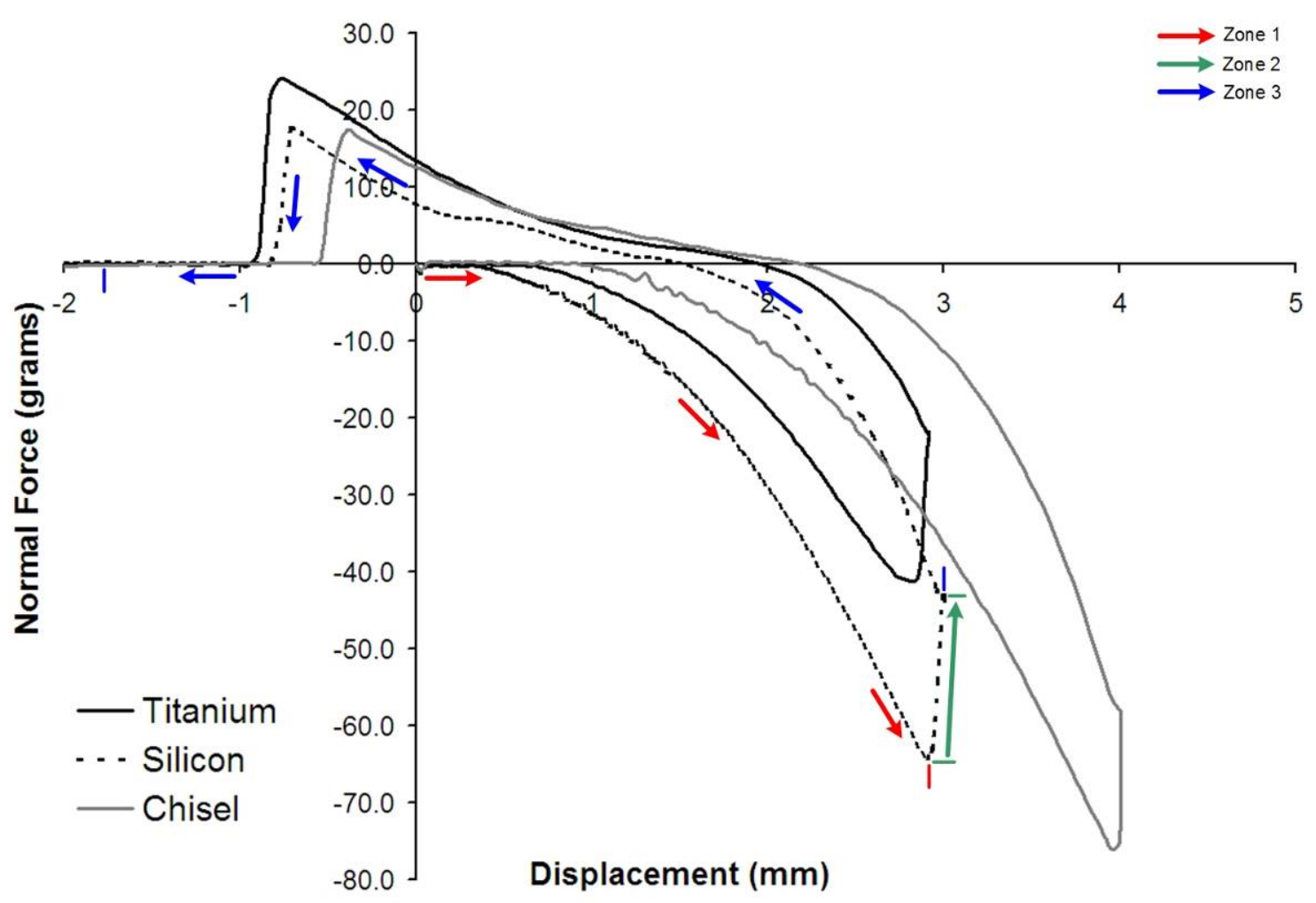

Figure 46. Normal force versus displacement plot for all microtack designs in the fiber reinforced silicone rubber gasket material 
Figure 47 exhibits the average displacement required for insertion and removal, respectively, for all the experiments for each microtack design. The average maximum displacement required for insertion of the titanium microtack was $2.81 \pm 0.03 \mathrm{~mm}$; this was $11.5 \%$ less than the silicon chisel tip design, and $8.5 \%$ less than the silicon pointed tip microtack. The average maximum displacement required for removal of the titanium microtack was $0.78 \pm 0.07 \mathrm{~mm}$, which was $8.8 \%$ greater than the silicon chisel tipped, and $24.7 \%$ greater than the silicon pointed tip microtack. The results illustrate that the titanium microtack requires less displacement for insertion and more displacement for removal than both the silicon microtack designs. Individual microtack results are included in Appendix IV.

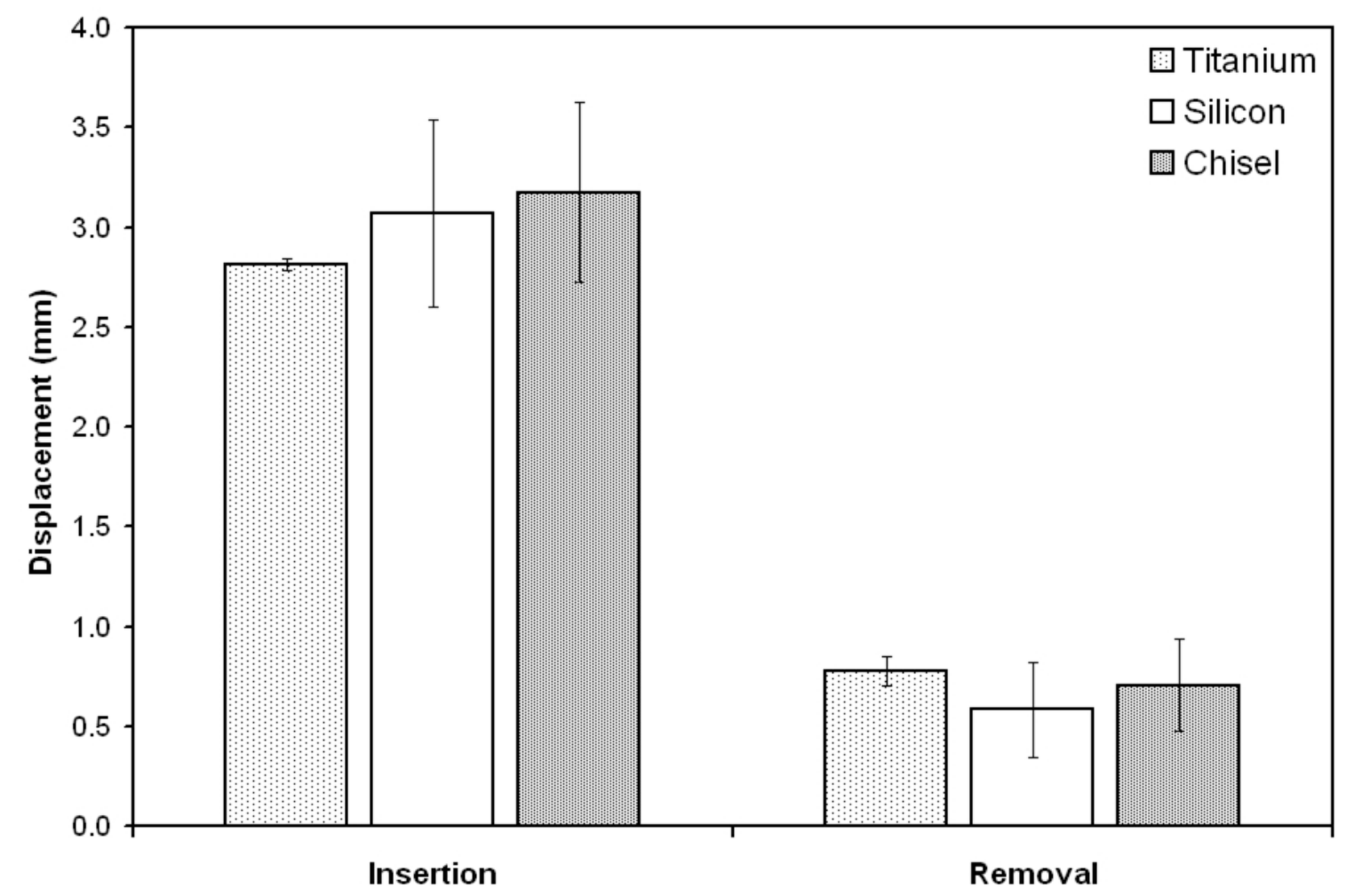

Figure 47. Average displacement required for insertion and removal for the microtacks designs in the fiber reinforced silicone rubber gasket material 
An alternative quantitative value for comparing the force data between microtack designs is the total force. The highlighted regions in Figure 48 shows the area under the force curve included in the calculation of the total force, specifically from 0 grams of load to the maximum value for insertion (red) and removal (blue). These regions were used for ease of calculation within MathCAD.

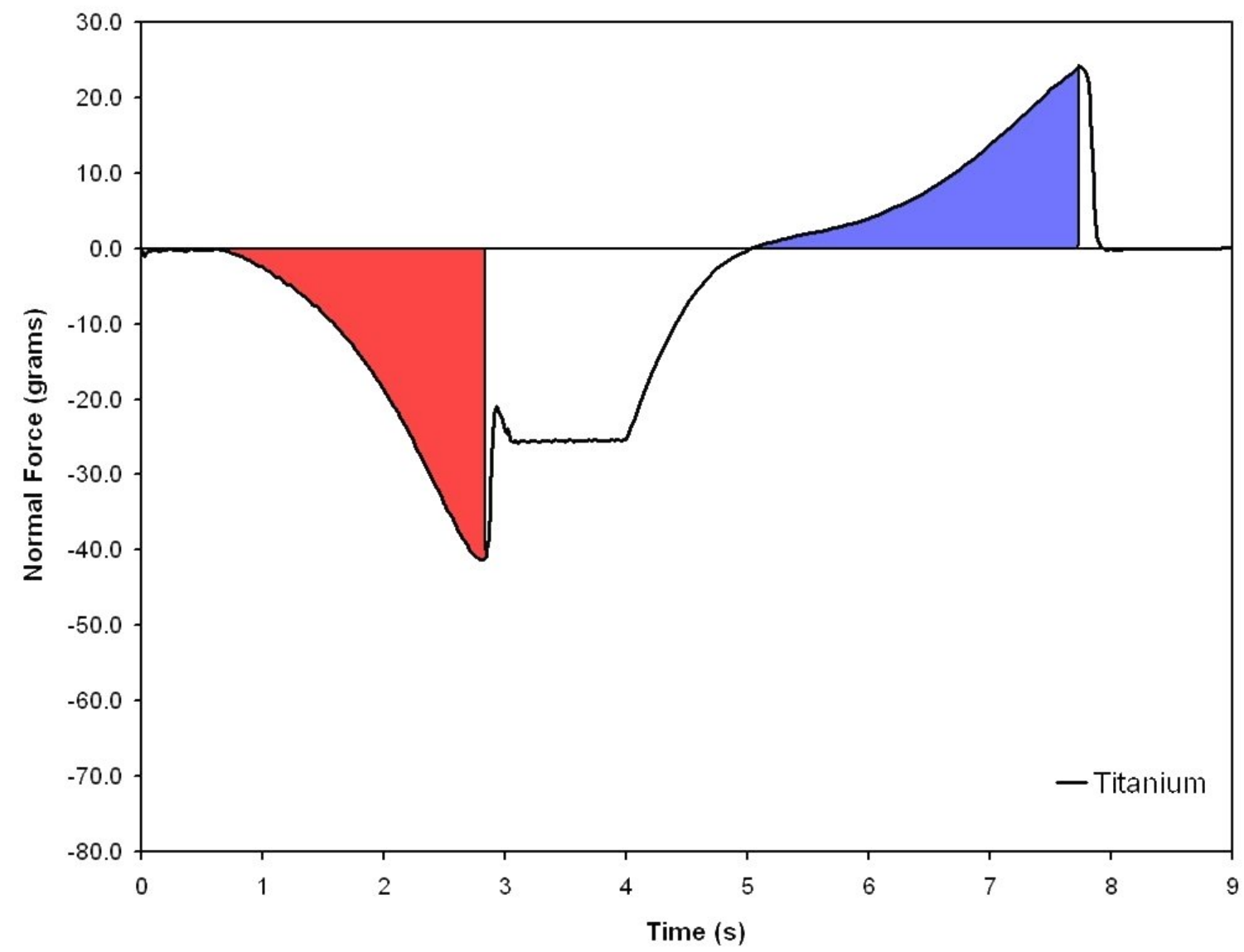

Figure 48. Normal force versus time plot illustrating how total force was calculated

Figure 49 summarizes the average total insertion and removal forces, respectively, for all the experiments for each microtack design. The total insertion force for the titanium tack was the lowest of the three designs and it also had the greatest total removal force, while the silicon pointed tip tack requires greater insertion force than the titanium and less than 
silicon chisel tip. The total force for removal for the silicon pointed tip was approximately the same as the silicon chisel tip, due to the similar barb design. The average total insertion force correlates well with the maximum insertion force results in that the titanium microtack $(35.78 \pm 4.82$ grams $)$ was found to be significantly lower $\left(\alpha_{\text {insertion }}<<0.001\right)$ than the silicon chisel tip $(51.1 \%$ higher $)$ and silicon pointed tip (46.0\% higher) microtacks. Similarly, the average total removal force of the titanium microtack $(24.60 \pm 2.26$ grams $)$ was significantly higher $\left(\alpha_{\text {removal }}<<0.001\right)$ than the silicon chisel tip (37.2\% lower) and silicon pointed tip (40.5\% lower) designs. Whereas the total insertion and removal forces for the chisel tip and pointed tip silicon microtack designs were found to be significantly different from the titanium design. This further supported the acceptance of the study's hypotheses. Individual microtack results are included in Appendix IV. Thus, the total force results correspond well with the maximum insertion and removal force results reported earlier. 


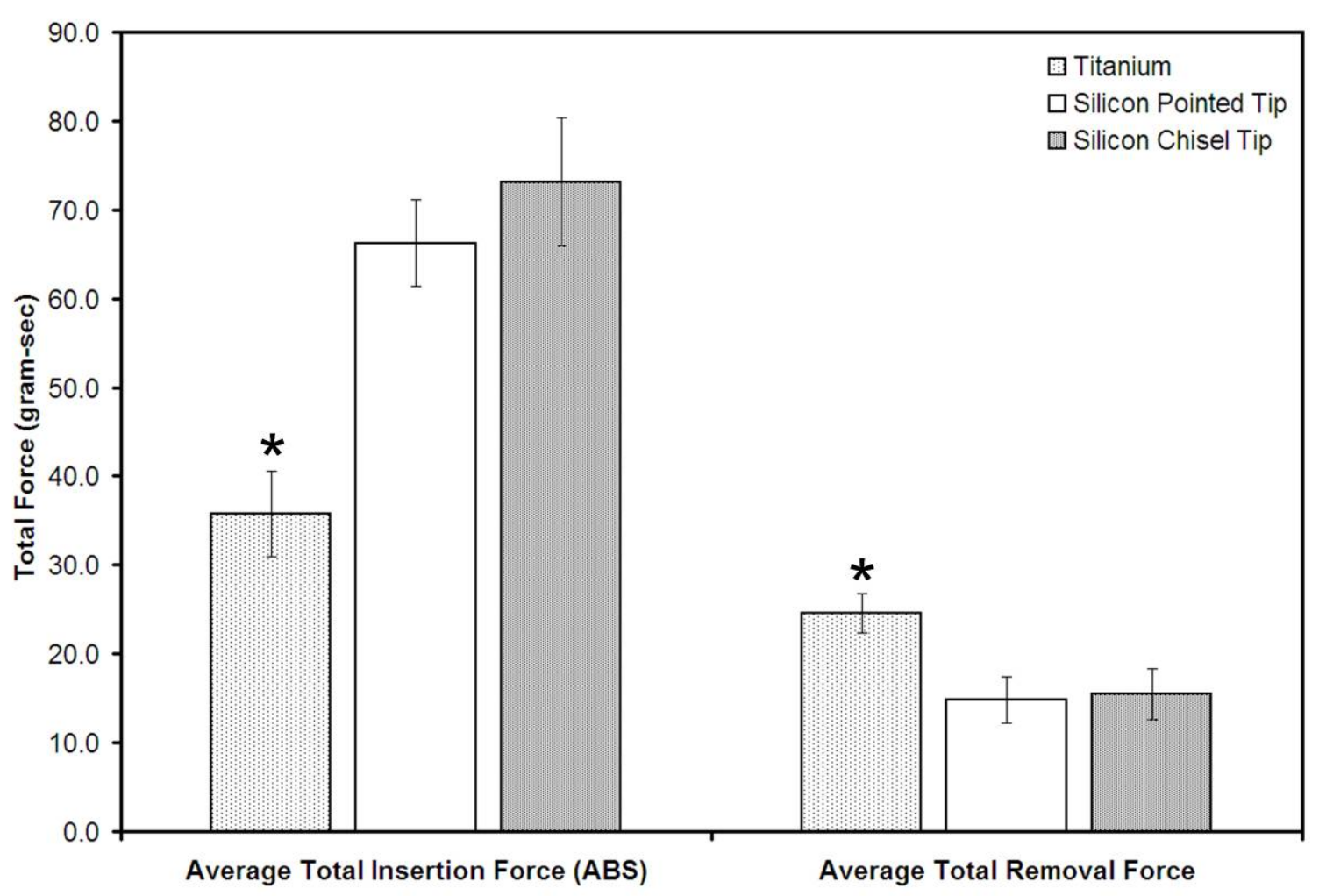

Figure 49. Average total insertion and removal force for the microtacks designs in the fiber reinforced silicone rubber gasket material. $\left({ }^{*} \alpha<0.001\right)$

Minimally invasive microtack insertion is desired, by doing so, damage to the retinal neural tissue will, theoretically, be low. Puncture geometries varied between microtack designs in the fiber reinforced silicone rubber. Figure 50 are representative stereomicroscope images of the insertion/removal points for each microtack design in the fiber reinforced silicone rubber material. In figure 50a, the intersection of the four markings indicates the initial penetration point and the four lines radiating from this point of intersection represents the fracture/crack propagation through the material until complete insertion was achieved. Figure 50b displays the point of insertion/removal of a silicon pointed tip microtack. The puncture geometry is similar to that of the titanium tack, however, the two base fracture lines are clearly defined compared to the Ti sample and the fracture lines are less evenly distributed in the radial direction. Figure 50c is the 
insertion/removal point for the silicon chisel tack. This puncture geometry is significantly larger and comparatively different than the two prior. The vertical line is the initial line of penetration. The other two lines are the fracture propagation due the parallel geometry of the chisel microtack.

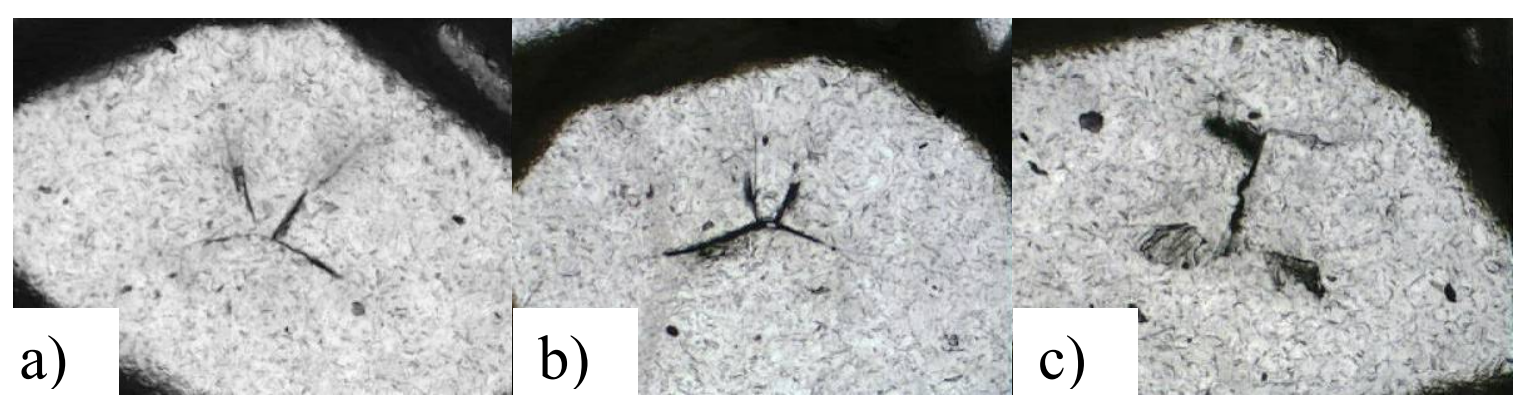

Figure 50. Stereomicroscope images of insertion/removal points of a) titanium, b) silicon pointed tip, and c) silicon chisel tip microtacks in the fiber reinforced synthetic rubber gasket material

\subsubsection{Characterization of the Microtack Performance in a Porcine Eye}

Actual ocular tissue experiments were performed in porcine (pig) eyes. Each microtack was inserted less than $6 \mathrm{~mm}$ from the optic nerve into the scleral tissue externally into the back of the eye. The ease of testing and repeatability of the force experiments drastically decreased with the porcine eye specimens. These two issues stemmed from several possible components encountered before and during the porcine eye testing.

A total of 4 microtacks of each design were inserted into porcine eyes. Figure 51 illustrates the average insertion and removal forces respectively, for all the experiments for each type of microtack design. The results differ from that of the synthetic rubber material. With this mode of experimentation, the silicon chisel tip microtack required less insertion force than both the silicon pointed tip microtack and the titanium sharp tip 
microtack; however, the titanium sharp tip tack still required the greatest amount of removal force among the three. The average maximum insertion force of the titanium microtack was $450.78 \pm 124.85$ grams; this was $21.7 \%$ more than the silicon chisel tipped, and $5.5 \%$ more than the silicon pointed tip microtack. The average maximum removal force for the titanium sharp tip microtack was $39.93 \pm 17.00$ grams; this was $15.1 \%$ more than the silicon chisel tipped, and $32.9 \%$ more than the silicon pointed tip microtack. Notwithstanding, none of the three microtack designs were significantly different than another for the amount of insertion or removal force. Individual microtack results are included in Appendix IV.

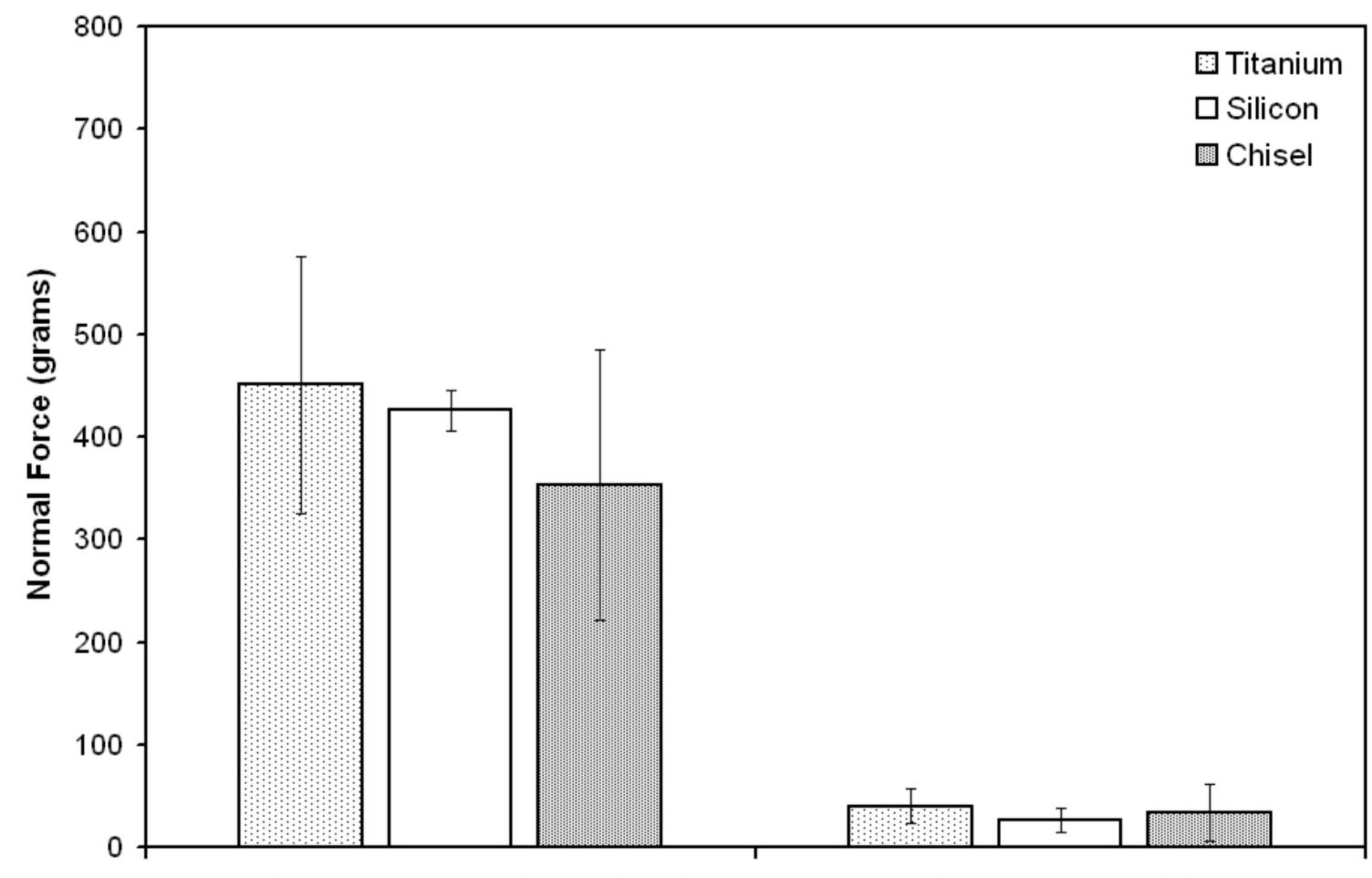

Maximum Insertion Force (ABS) Maximum Removal Force

Figure 51. Average maximum insertion and removal forces for each microtack design in the porcine ocular tissue

Figure 52 illustrates the average displacement required for insertion and removal, respectively, for all ocular experiments using each type of microtack design. These 
results also differ from the synthetic rubber material results. The silicon pointed tip microtack required less displacement for insertion than both the silicon chisel tip microtack and the titanium sharp tip microtack. The titanium sharp tip and silicon chisel tip microtack were within $0.50 \%$ of one another. The average displacement required for insertion of the silicon pointed microtack was $5.650 \pm 0.698$ grams, which was $21.9 \%$ less than the silicon chisel tip tack and $21.6 \%$ less than the titanium microtack. The average maximum displacement required for removal of the titanium microtack was $1.810 \pm 1.80$ grams, which was $5.8 \%$ more than the silicon chisel tip and $73.8 \%$ more than the silicon pointed tip microtacks. However, again, neither the displacements during insertion nor removal were significantly different between the microtack designs. Individual microtack results are included in Appendix IV.

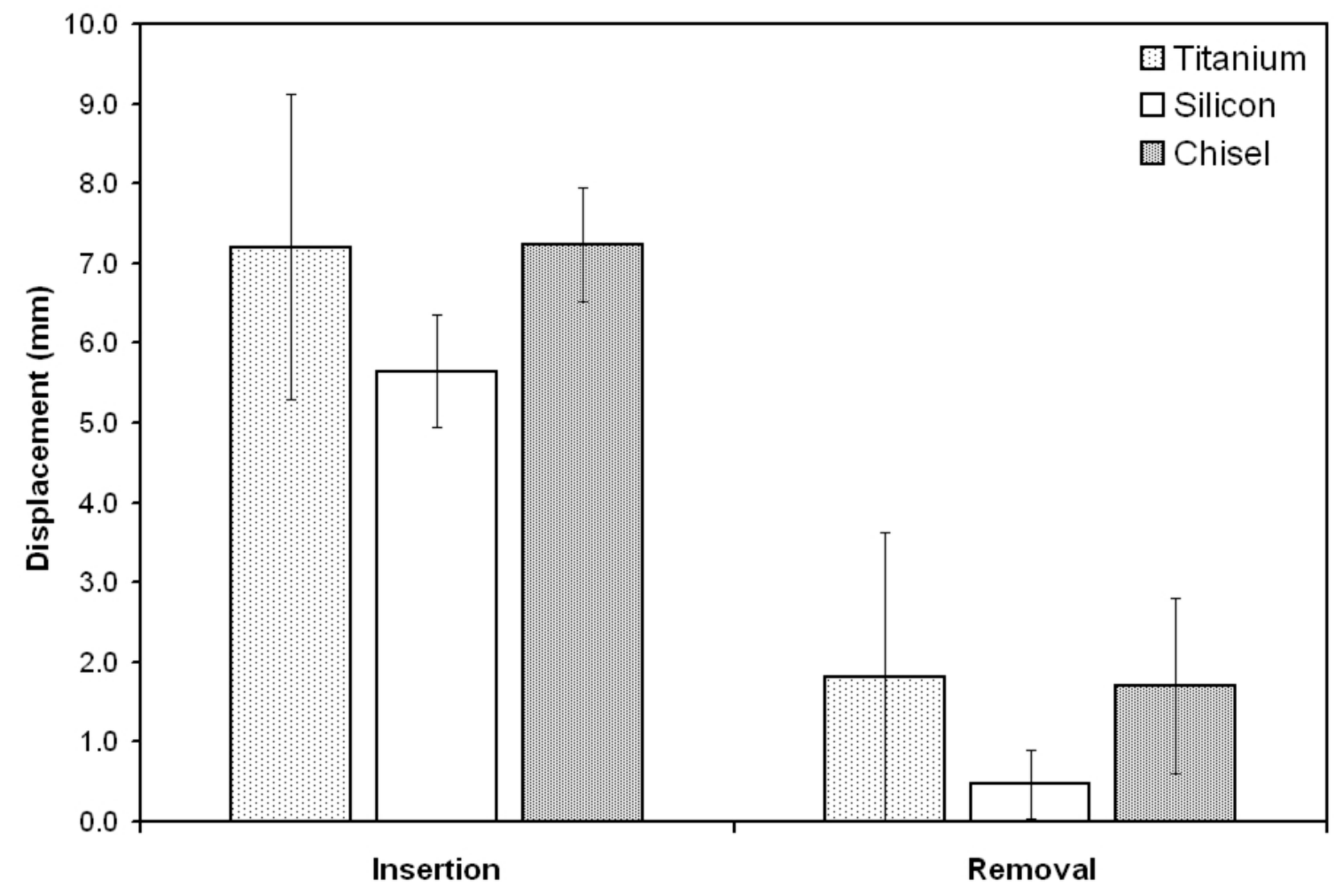

Figure 52. Average displacement required for insertion and removal of the different microtack designs in the porcine ocular tissue 
Figure 53 shows the average total insertion and removal forces respectively, for all the ocular experiments for each type of microtack design. The silicon chisel tip microtack required less total insertion force than both the silicon pointed tip microtack and the titanium sharp tip microtack. The average total insertion force of the silicon chisel microtack was $492.29 \pm 218.23$ grams; this was $13.3 \%$ less than the titanium microtack and $9.7 \%$ less than the silicon pointed tip microtack. The titanium tack required the most removal force of the three microtack designs with an average total removal force of $56.08 \pm 44.67$ grams for the titanium microtack, which was $1.8 \%$ more than and $61.4 \%$ less than the silicon chisel tip and the silicon pointed tip microtacks, respectively. Again, no significant differences were found between the three microtack designs in the biological tissue experiments for the total insertion and removal forces. Individual microtack results are included in Appendix IV. 


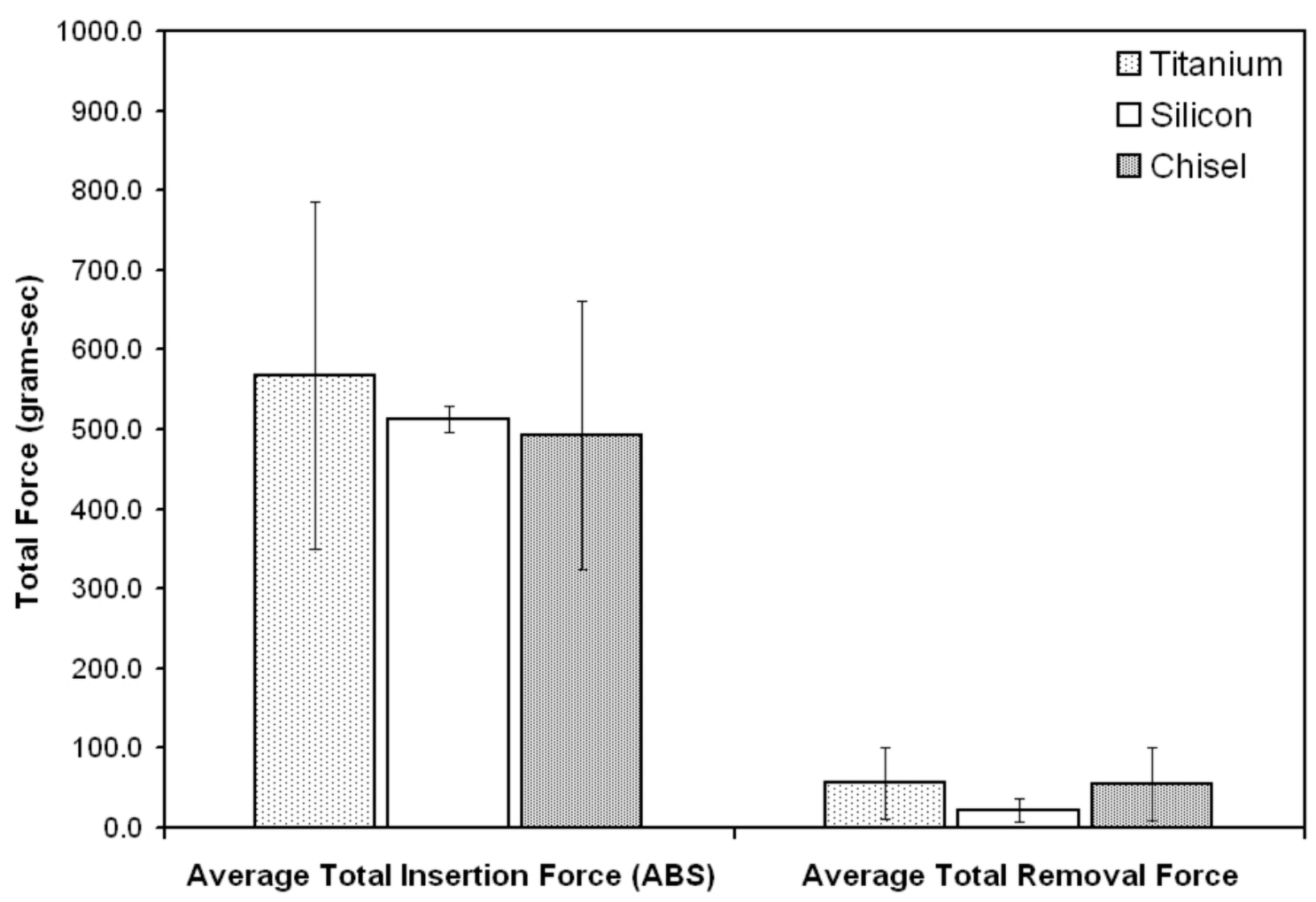

Figure 53. Average total insertion and removal force for the microtacks designs in the porcine ocular tissue

\subsubsection{Discussion of Variance Between Samples}

Several issues were of concern during and after the completion of the ocular experiments. Repeatability between measurements was a major concern compared to the rubber experiments. The primary reason for the differences between the sample results is that the fiber reinforced silicone rubber is a man made material with near homogeneous material properties, whereas the porcine ocular tissue varies from sample-to-sample since biological tissue obtained from multiple animals will vary in material property uniformity due to each biological tissue sample having different amounts of collagen and cell density in the tissue based on age and gender of the animal. In addition, the length of time between removing the tissue from the animal and experimental testing would affect the amount of cross-linking between the collagen fibers and number of cells undergoing 
apoptosis; thereby, altering the stiffness between the biological tissue samples. Similar structural variances also exist throughout the tissue, side-to-side, and from animal-toanimal. Unfortunately, all three tack designs were not tested in the same porcine tissue since alterations to the tissue during testing made it impossible

The biomechanical properties of the eye, primarily the sclera, are largely associated with the collagen content. Collagen is a protein that is the basic structural element for soft and hard tissues, including the sclera, the principal load bearing framework of the eye. Scleral tissue contains approximately $90 \%$ collagen by weight [Rada 2005]. With this high percentage, scleral tissue is highly sensitive to the changes in collagen synthesis. The biomechanical properties of the sclera depend largely on the interactions and crosslinking of collagen. These interactions and alterations are influenced by, to name a few, aging [Diamant 1972, Fung 1993], vision problems such as myopia and hyperopia (nearsightedness and farsightedness, respectively) [Rada 2005], vitreous humor liquefaction [Ihanamaki 2004], and induced collagen crosslinkage [Wollensak 2004]. All of the stated alterations cause an increase in the crosslinking of collagen, which increases the visco-elasticity of the sclera, making it a more rigid structure. Wollensak et al. (2004) reported that treating human and porcine scleral tissue with Glutaraldehyde induced collagen crosslinkage and significantly increased the Young's Modulus by $122 \%$ and $817 \%$, respectively. The crosslinkage of the porcine tissue is probably the foremost contributor to the lack of repeatability in these experiments.

The porcine eyes were removed from older age pigs and several eyes were used for testing. The donated eyes due to the age of the pig had already begun to undergo 
vitreous liquefaction. The percentage of liquefaction differs between each eye; this places another variable into the equation, creating more uncertainty in the measurements.

Tissue preservation also influenced the material properties of the eyes. Specimens were used in experiments typically within 2 to 3 days of pickup. They were preserved in a saline solution for that time. Collagen crosslinkage could have occurred if the preservation process was not optimized, creating an increase of uncertainty in the measurements.

Breakage and deformation of the silicon and titanium microtacks were a key problem in the ocular force measurement experiments. These buckling failures were primarily due to the increased visco-elasticity of the porcine tissue. One out of four silicon chisel tip microtacks and two of the four silicon pointed microtacks broke upon insertion into the ocular tissue, while one of four titanium microtacks bent during insertion. Table 7 displays the loads at which these microtacks failed along with the analytical results. The titanium and silicon pointed microtacks failure loads fall within the designated analytical range stated earlier. The silicon chisel tip microtack failed due to a much lower load than the analytical results. This could be due to an over tightening of the micro-clamp, possibly cracking the brittle silicon before insertion. Figures 54 through 56 are SEM images of each of the microtack designs demonstrating the various modes of failures for the tacks.

Table 7. Actual buckling failure corresponding to analytical data.

\begin{tabular}{|c|c|c|c|c|}
\cline { 3 - 5 } \multicolumn{1}{c|}{} & \multicolumn{3}{c|}{ Analytical } \\
\cline { 2 - 5 } \multicolumn{1}{c|}{} & Failure & Bending & Johnson & Secant \\
\hline Titanium & 539.99 & 222.99 & 840.12 & 211.9 \\
\hline \multirow{2}{*}{ Si Point } & 539.60 & 248.47 & 988.78 & 237.53 \\
\cline { 2 - 5 } & 513.84 & & & \\
\hline Si Chisel & 161.41 & 993.88 & 988.78 & 624.4 \\
\hline \multicolumn{4}{|c|}{$* *$ Units $=$ grams $_{\text {force }} * *$} \\
\hline
\end{tabular}




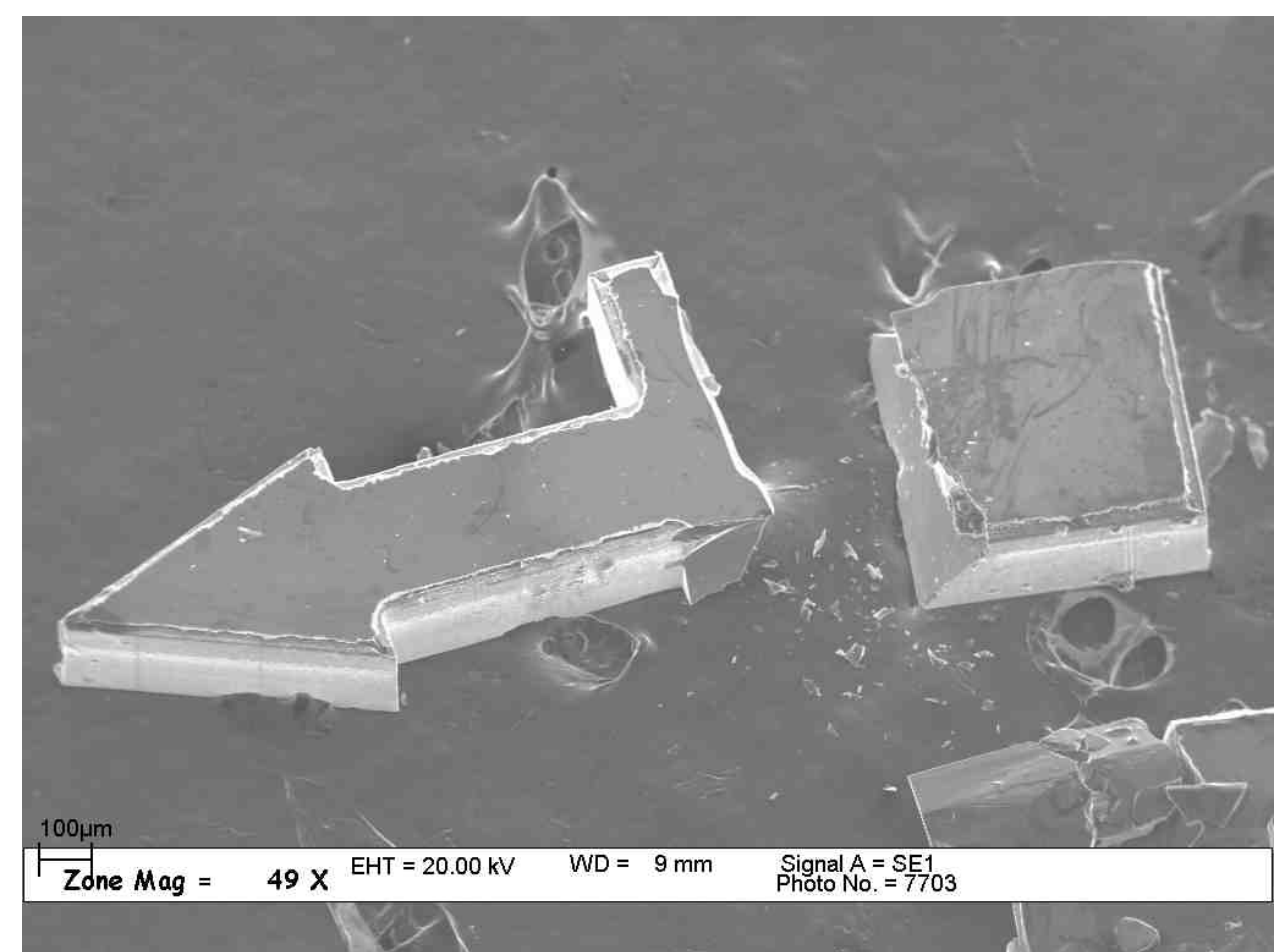

Figure 54. SEM image of the failure mode of the silicon chisel tip microtack

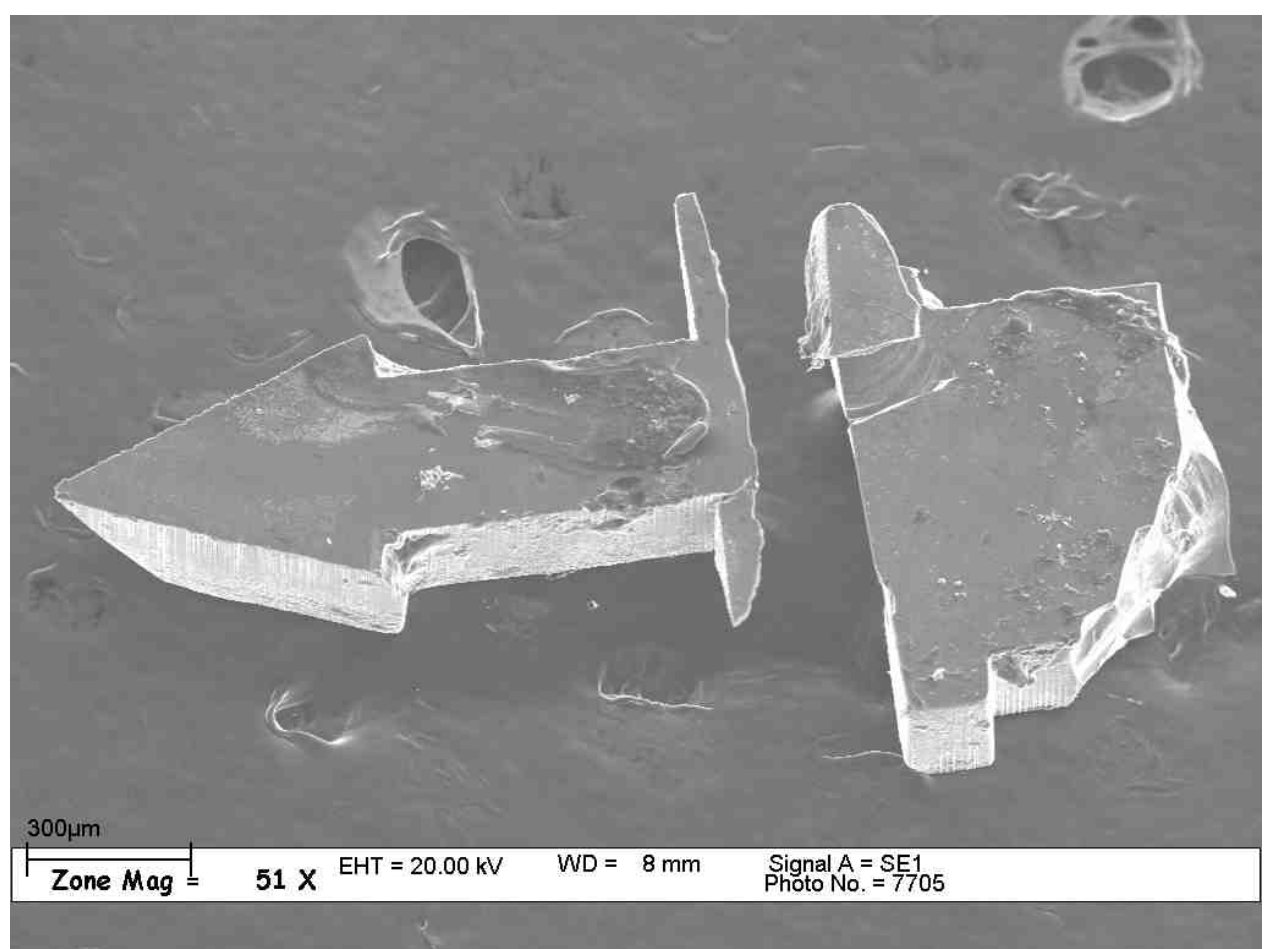

Figure 55. SEM image of the failure mode of the silicon pointed tip microtack (Note: the tack was imaged upside down, hence the surface of the pointed tip is not shown.) 


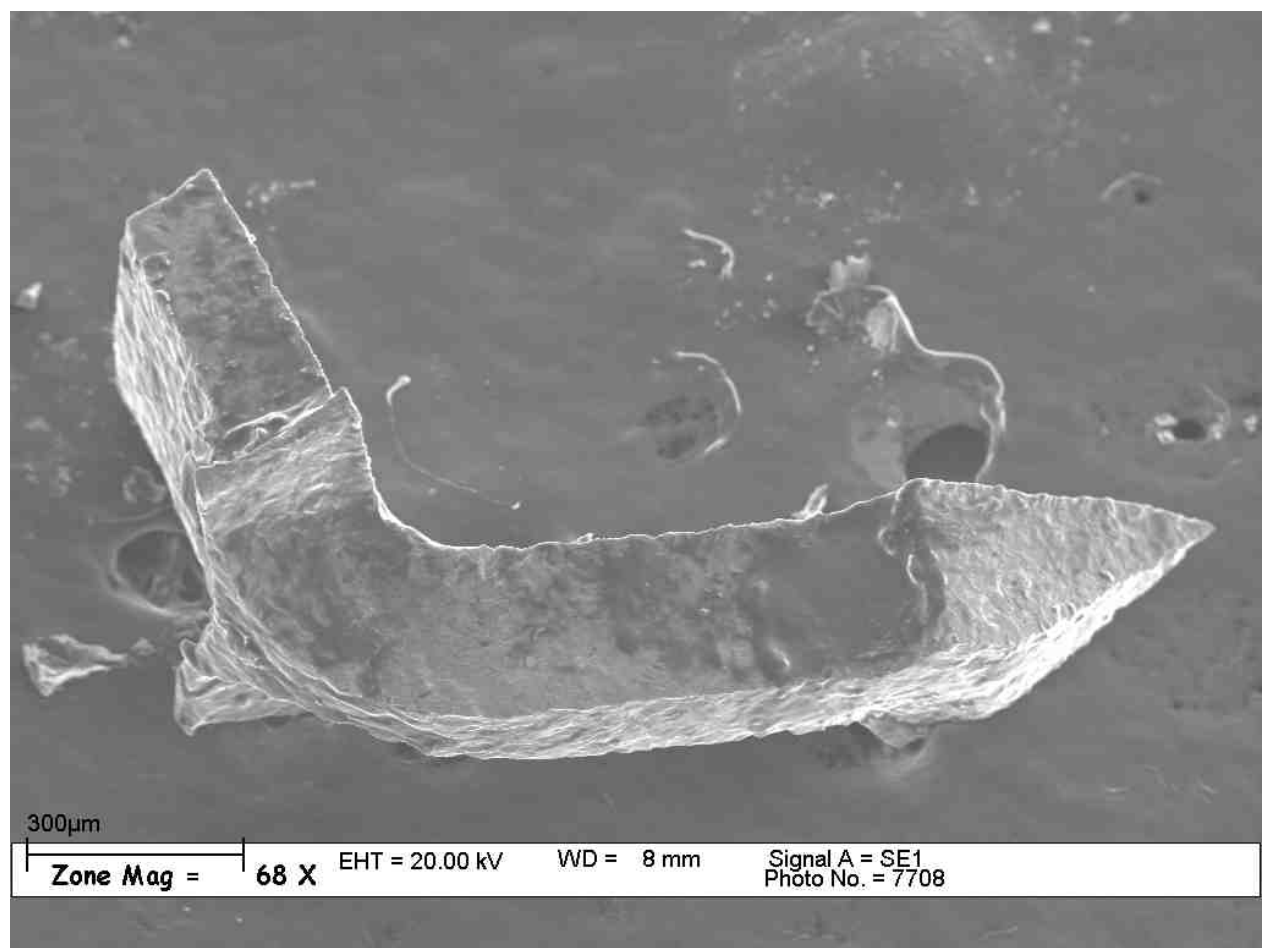

Figure 56. SEM image of the failure mode of the titanium microtack

The figures above portray the ultimate difference between the silicon and titanium microtacks. If either of the silicon microtack designs were used for actual clinical surgeries and was to fail, fragments of silicon would be dispersed throughout the vitreous and around the retinal region, leaving the patient in a worse situation. Whereas, if the titanium microtack were to fail, it would simply bend, giving the clinician an easy opportunity to remove it and insert a new one. 


\section{CONCLUSIONS}

Retinal microtacks were successfully fabricated out of traditional silicon material and also out of a more biocompatible and ductile titanium material, using two different microfabrication procedures; DRIE with wet anisotropic etching and ultra-high-precision micromilling techniques, respectively. Metrology was performed to verify the accuracy of both fabrication methods.

Force measurement experiments were successfully executed. Results of the tests using fiber reinforced synthetic rubber gasket material proved the stated hypotheses, where the titanium microtack required less insertion force and more removal force than both silicon microtack designs. The results were also quite repeatable, with minimal deviation. On the other hand, the tests carried out in the porcine eye tissue did not coincide with the stated hypothesis, had poor repeatability and relatively high deviation. This was primarily due to the experimental plan. Further studies should be performed to determine possible flaws in the experimental plan for testing the microtacks in actual ocular tissue. The failures of all three microtack designs within the ocular tissue were unexpected but also determined that the titanium was the best choice of the design types. 


\section{RECOMMENDATIONS}

Several recommendations are listed below that may assist in the ongoing progress in determining optimal microtack design for retinal prosthesis fixation.

- Vary insertion/removal velocity to determine whether this has any effect on loading scenarios.

- Fabricate microtacks out of higher strength materials (i.e. titanium alloys) that have similar biocompatibility characteristics.

- Design an insertion tool that will consistently grip microtacks of various geometries that can also by used by clinicians during surgeries.

- Improve upon experimental plan for testing the microtacks within ocular tissue. By designing a fixture that would replicate the compliance and cushioning of the socket which the eye ball rests. By doing this, the tacks could be inserted within the eye, in the actual position it would be placed.

- Implement eye testing within a test chamber consisting of a buffered saline solution heated to human body temperature of $98.6^{\circ} \mathrm{F}$. This would possibly emulate living tissue characteristics.

- Implant various microtack designs of assorted biocompatible materials into living subjects to determine which material has better tissue response over a planned length of time. 


\section{REFERENCES CITED}

Abrams, B.W., Williams G.A., Neuwirth, J., McDonald, H.R., 1986, “Clinical results of titanium retinal tacks with pneumatic insertion," American Journal of Ophthalmology, 102(1) 13-9

Ambati, J., Ambati, B.K., Yoo, H.S., Ianchulav, S., Adamis, A.P., 2003, “Age-Related Macular Degeneration: Etiology, Pathogenesis, and Therapeutic Strategies," Survey of Ophthalmology, 48(3) 257-293

Anderson, D.H., Mullins, R.F., Hageman, G.S., Johnson, L.V., 2002, "Role for Local Inflammation in the Formation of Drusen in the Aging Eye," American Journal of Ophthalmology, 134(3) 411-431

Barbucci R., 2002, Integrated Biomaterials Science, New York, Kluwer Academic/Plenum Publishers

Beer, F.P., Johnston, E.R., 1992, Mechanics of Materials, Second Edition, New York, McGraw-Hill, Inc,

Brunette D.M., Tengvall P., Textor M., Thomsen P., 2001, Titanium in Medicine, Material Science, Surface Science, Engineering, Biological Responses and Medical Applications, Berlin, Springer

Burke, J.M., McDonald, H.R., Neuwirth, J., Lewandowski, M., 1987, “Titanium retinal tacks with pneumatic insertion. Histologic evaluation in rabbits," Arch. Ophthalmology, $105(3)$

Bzostek, A., Kumar, R., Diaz, L., Mukta, S., Anderson J.H., Taylor, R.H., 1999, "Force vs. Deformation in soft tissue puncture," Submitted for consideration to MICCAI'99, \#196

Cambron S.D., Keynton R.S., Franco-Sarabia J.R., Crain M.M., Shire D.B., Walsh K.M., Naber J., Kaplan H., and Rizzo J., 2003, "Design and Fabrication of Microtacks for Retinal Implant Applications," Proc. IMECE, 41572

Chow, A.Y., 1990, U.S. Patent 5,024,223

Chow, A.Y., 1993, "Electrical stimulation of the rabbit retina with subretinal electrodes and high density microphotodiode array implants," Investigative Ophthalmology and Visual Science, 34835 (Abstract)

Chow, A.Y., Chow, V.Y., Packo, K.H., Pollack, J.S., Peyman, G.A., Schuchard, R., 2004

"The Artificial Silicon Retina Microchip for the Treatment of Vision Loss From Retinitis Pigmentosa” Arch Ophthalmol., 122:460-469 
Dally W.D., Riley, W.F., McConnell, K.G., 1993, Instrumentation for Engineering Measurements, Second Edition, Wiley

Dee K.C., Puleo D.A., Bizios R., 2002, An Introduction to Tissue-Biomaterial Interactions, Hoboken, John Wiley \& Sons, Inc.

De Juan E Jr, McCuen, B.W., Machemer, R, 1987, "Mechanical retinal fixation using tacks," Ophthalmology, 94(4) 337-40

De l'Aune, WR, and Williams, MD. 2001, "Outcome assessment of the rehabilitation of the visually impaired," $\underline{C 1776 R}$

Diamant, J., Keller, A., Baer, E., Litt, M., Arridge, R., 1972, "Collagen; ultrastructure and its relation to mechanical properties as a function of ageing," Proc. R. Soc. Lond. B. $180,293-315$

Dobelle, W.H., 2000, "Artificial Vision for the Blind by Connecting a Television Camera to the Visual Cortex," ASAIO Journal,

Frick, T.B., Marucci, D.D., Cartmill, J.A., Martin, C.J., Walsh, W.R., 2001, "Resistance forces acting on suture needles," Journal of Biomechanics, 34 1335-1340

Friedrich C. and Vasile M., 1996, "Development of the Micromilling Process for High Aspect Ratio Microstructures," Journal of Microelectromechanical Systems, 5(1) 33-38.

Friedrich C. and Vasile M., 1996, "The Micromilling Process for High Aspect Ratio Microstructures," Microsystems Technologies, 2(3) 144-148.

Fung, Y., 1993, Biomechanics; Mechanical Properties of Living Tissues, New York, Springer

Gekeler, F., Schwahn, H., Stett, A., Kohler, K., Zrenner, E., 2001, “Subretinal microphotodiodes to replace photoreceptor-function. A review of the current state," $\underline{\text { Les }}$ Seminaires Ophthalmologiques d'IPSEN, tome 12,

Gerding, H., Taneri, S., Benner, P., Thelen, U., Uhlig, C.E., Reichelt, R., 2001, "Successful long-term evaluation of intraocular titanium tacks for the mechanical stabilization of posterior segment ocular implants," Materialwissenschaft und Werkstofftechnik, 32(12) 903-912

Gupta, N., Brown, K.E., Milam, A.H., 2003, "Activated microglia in human retinitis pigmentosa, late-onset retinal degeneration, and age-related macular degeneration," Experimental Eye Research, 76 463-471

Guyton, A.C., Hall, J.E., 1996, Textbook of Medical Physiology, Philiadelphia, W.B. Saunders Co. 
Hecht, F., Shiel, W., 2003, Webster's New World ${ }^{\mathrm{TM}}$ Medical Dictionary, 2nd Edition, Wiley

Humayun, MS, de Juan, E, et al., 1999, "Morphometric Analysis of the extramacular retina from postmortem eyes with retinitis pigmentosa," Inv. Ophthalmol. Vis. Sci.,; 40 143-148.

Humayun, M.S., 2001, “Intraocular Retinal Prosthesis,” Tr. Am. Ophth. Soc. 99 271-300

Ihanamaki, T., Pelliniemi, L., Vuorio, E., 2004, "Collagens and collagen-related matrix components in the human and mouse eye," Progress in Retinal and Eye Research, 23 403-434

Jackson, G.R., Owsley, C., Curcio, C.A., 2002, "Photoreceptor degeneration and dysfunction in aging and age-related maculopathy," Ageing Research Reviews, 1381-396

Juvinall, R.C., Marshek, K.M., 2000, Fundamentals of Machine Component Design, Third Edition, New York, John Wiley \& Sons, Inc.

Kapit, W., Macey, R.I., Meisami, E., 1987, The Physiology Coloring Book, New York, HarperCollins Publishers, Inc.

Lichtenan R.F., 1997, WYKO NT-2000 Oprerator's Guide, V1.000, WYKO Corporation, Tucson, AZ

Majji, A., Humayun, M., Weiland, J., Suzuki, S., D’Anna, S., de Juan, E., 1999, “Longterm Histological and Electrophysiological Results of an Inactive Epiretinal Electrode Array Implantation in Dogs," Invest. Ophth. \& Vis. Sci., V40 N9, 2073-2081

Margalit, E., Fuji, G., Lai, J.C., Gupta, P., Chen, S., Shyu, J., Piyathaisere, D., Weiland, J.D., De Juan Jr., E., Humayun, M.S., 2000, "Bioadhesives for intraocular use," Retina, 20(5) 469

Margalit, E., Maia, M., Weiland, J.D., Greengerg, R.J., Fuji, G., Torres, G., Piyathaisere, D., O’Hearn, T.M., Liu, W., Lazzi, G., Dagnelie, G., Scribner, D.A., De Juan Jr., E., Humayun, M.S., 2002, "Retinal Prosthesis for the Blind," Survey of Ophthalmology, 47(4) 335-356

Maynard, E.M., 2001, “Visual Prosthesis,” Annu. Rev. Biomed. Eng. 3 145-168

Medeiros, NE and Curcio, CA. 2001, "Preservation of ganglion cell later neurons in agerelated macular degeneration," Invest. Ophthalmol. Vis. Sci., 42(3) 795-803.

Meyer, J., 2002, "Retina implant - a bioMEMS challenge," Sensors and Actuators A 97$98 \quad 1-9$ 
Michelson, R.P., 1985, U.S. Patent 4,628,933

Nisch, W., 1999, U.S. Patent 6,298,270 B1

Norton, R.L., 1998, Machine Design; An Integrated Approach, Second Edition, Prentice Hall

Park J.B., Lakes R.S., 1992, Biomaterials, An Introduction Second Edition, New York, Plenum Press

Peachey, N.S., Chow, A.Y., 1999, "Subretinal Implantation of Semiconductor-Based Photodiodes: Progress and Challenges," J. Rehabilitation and Development, 36(4) 371376

Puustjarvi, T.J., Terasvirta, M.E., 2001, "Retinal fixation of traumatic retinal detachment with metallic tacks: a case report with 10 years' follow up," Retina, 21(1) 54-6

Rada, J.A., Shelton, S., Norton, T., 2005, “The sclera and myopia," Exp. Eye Res., 82 $185-200$

Rapantzikos, K., Zervakis, M., Balas, K., 2003, "Detection and segmentation of drusen deposits on human retina: Potential in the diagnosis of age-related macular degeneration," Medical Image Analysis 7 95-108

Rizzo, JF and Wyatt, JL. 1997, "Prospects for a visual prosthesis," Neuroscientist,; 3 251-262.

Rizzo J.F., Wyatt J.L., Humayun, M., et al. 2001, "Retinal prosthesis: an encouraging first decade with major challenges ahead." Ophthalmol. 108 13-14

Rowe-Rendleman, C., Glickman, R.D., 2003, "Possible therapy for age-related macular degeneration using human telomerase," Brain Research Bulletin

Santos, A., Humayun, M., de Juan, E., Greenburg, R., Marsh, M., Klock I., Milam A., 1997, "Preservation of the inner retina in retinitis pigmentosa. A morphometric analysis," Arch. Ophthalmol. V115 N4

Schubert, M.B., Hierzenberger, A., Lehner, H.J., Werner, J.H., 1999, “Optimizing photodiode arrays for the use as retinal implants," Sensors and Actuators 74 193-197

Sharma, R.K., Ehinger, B., 1999, "Management of Hereditary Retinal Degenerations: Present Status and Future Directions," Survey of Ophthalmology, 43(5)

Shaw, M., 2005, Metal Cutting Principles, Second Edition, New York, Oxford University Press 
Shire D. B., Rizzo J.F., Lowenstein J., 2002, "Micromachined Tacks to Attach a Prosthesis to the Retina," Invest. Ophthalmol. Vis. Sci. 43: E-Abstract 4482

Shire, D.B. and Rizzo, J.F., 2002, "Microfabrication Initiatives at the VA Center for Innovative Visual Rehabilitation (CIVR)," Proc. 2nd Joint EMBS/BMES Conf. 23992400 .

Stone, J, Barlow, WE, Humayun, MS, de Juan, E, and Milam, AH. 1992, "Morphometric analysis of macular photoreceptors and ganglion cells in retinas with retinitis pigmentosa," Arch. Ophthalmol.; 110:1634-1639.

TA Instruments, 2003, RSA III, Rheometric Series User Manual, New Castle, DE, TA Instruments-Waters LLC

Tassicker, G.A., 1954, U.S. Patent 2,760,483

Taylor, H.R., 1997, Data Acquisition for Sensor Systems, London, Chapman and Hall

Tezel, T., Bora, N., Kaplan, H., 2004, "Pathogenesis of age-related macular degeneration," TRENDS in Mol. Med., V10 N9

Tortura, G., 1997, Introduction to the Human Body, The Essentials of Anatomy and Physiology, Fourth Edition, RR Donnelley \& Sons Co.

Vasile M., Friedrich C., Kikkeri B,, McElhannon R., 1996, "Micrometer-scale machining: Tool fabrication and initial results," Precision Engineering 19, 180-186

Wallensak, G., Spoerl, E., 2004, "Collagen crosslinking of human and porcine sclera," $\underline{J}$ Cataract Refract. Surg., 30 689-695

Zrenner E., Stett, A., Weiss, S., Aramant, R.B., Geunther, E., Kohler, K., Miliczek, K.D., Seiler, M.J., Haemmerle, H., 1999, "Can subretinal microphotodiodes successfully replace degenerated photoreceptors?" Vision Research, 39(15) 2555-2567 


\section{APPENDIX I}

PMAC MACHINING CODE 


\section{PMAC program for micro clamp jaw machining process}

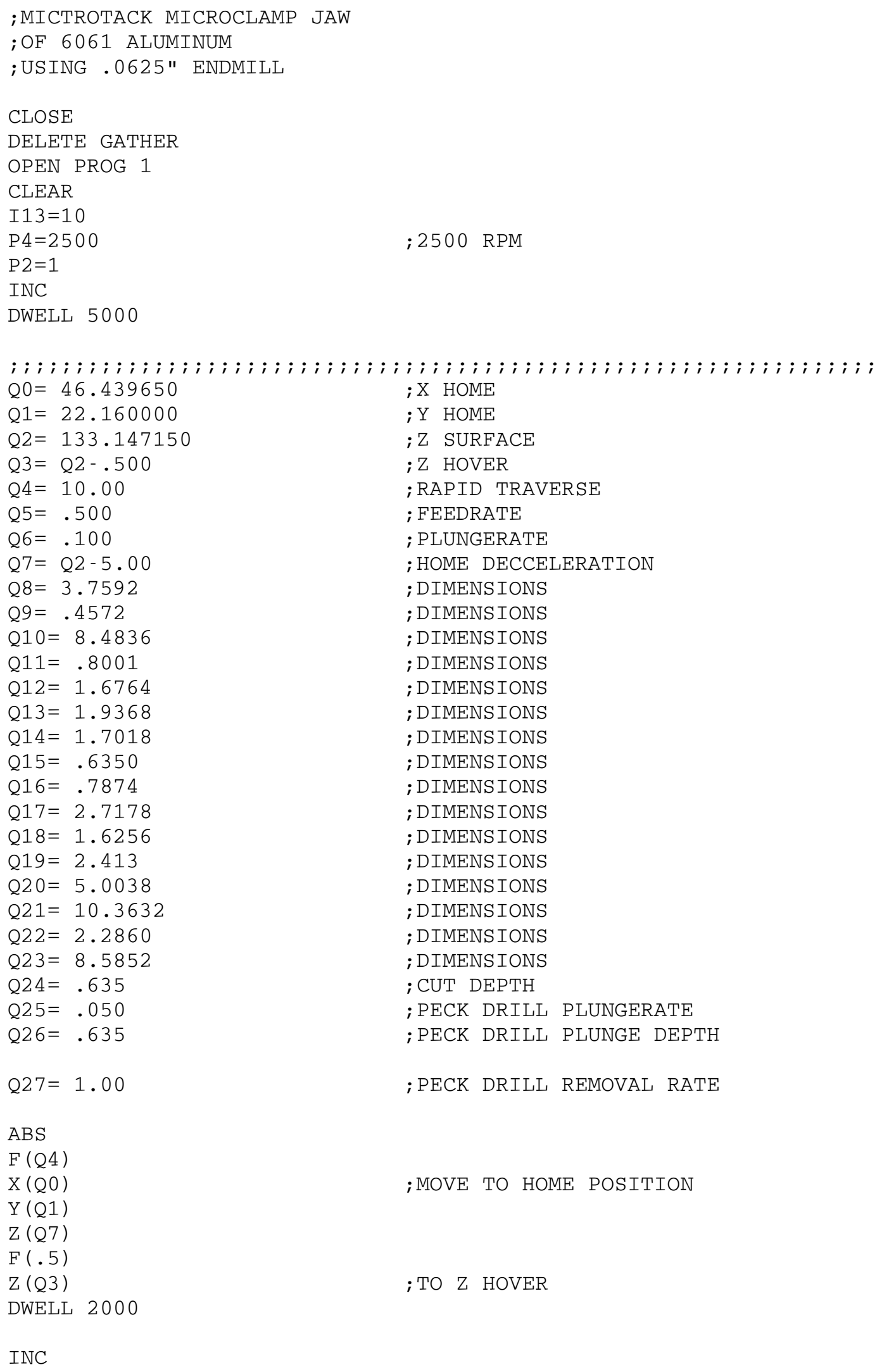




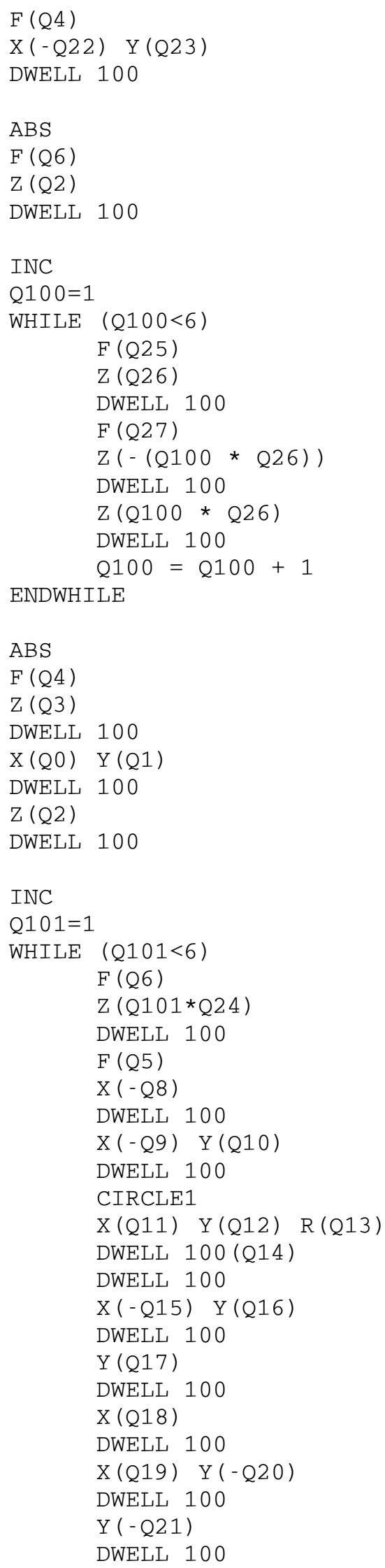




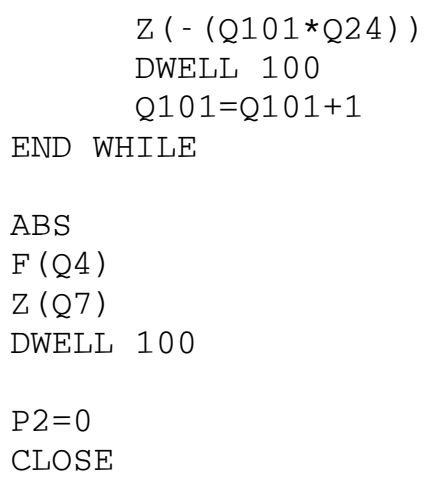

\section{PMAC program for titanium microtack machining processes}

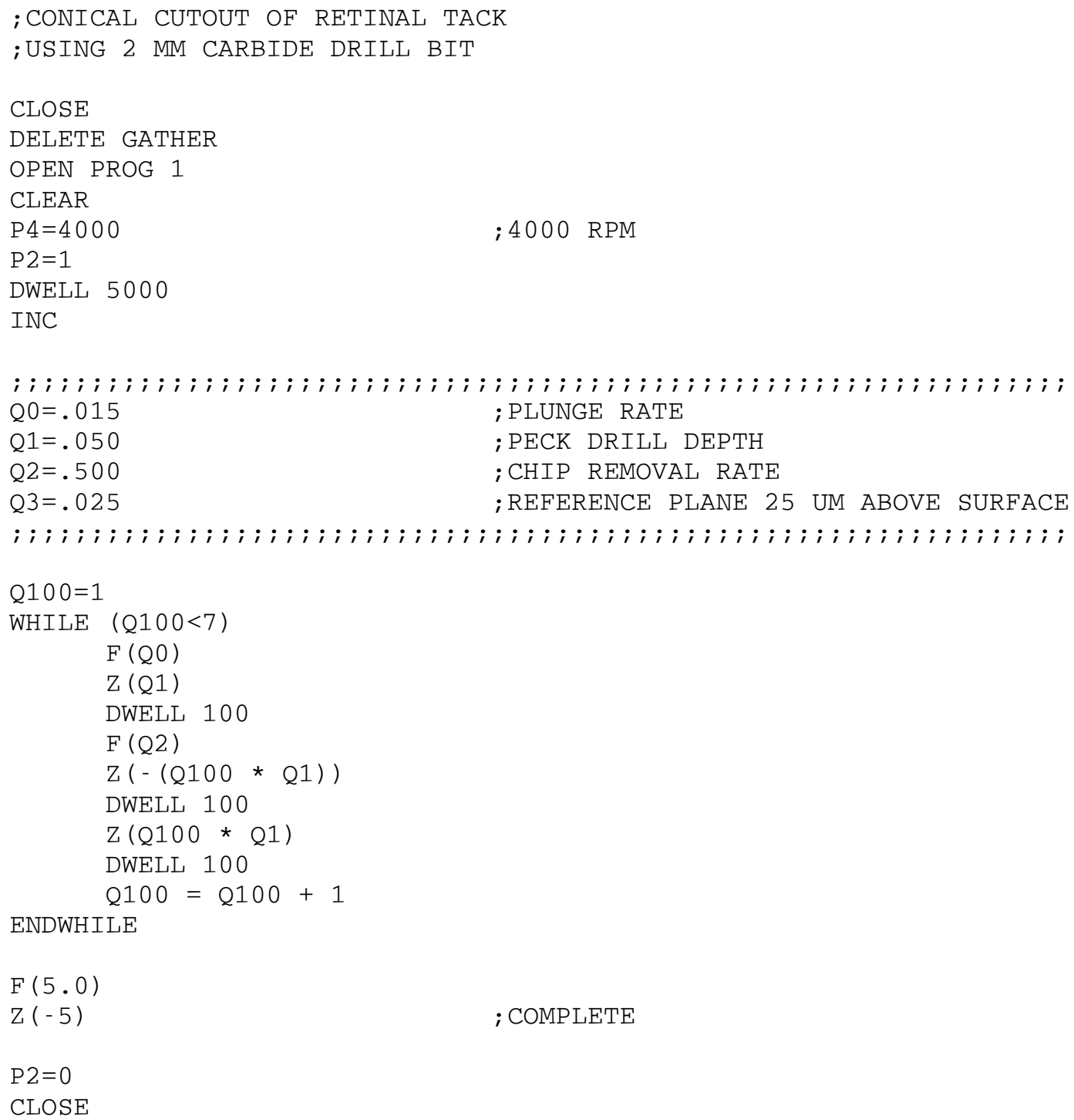


; RETINAL TACK PROCESS

; PRIOR ROUGH DRILLING OF CONE USING 2MM CARBIDE DRILL ;FINISH PASS AT END USING 150UM ENDMILL

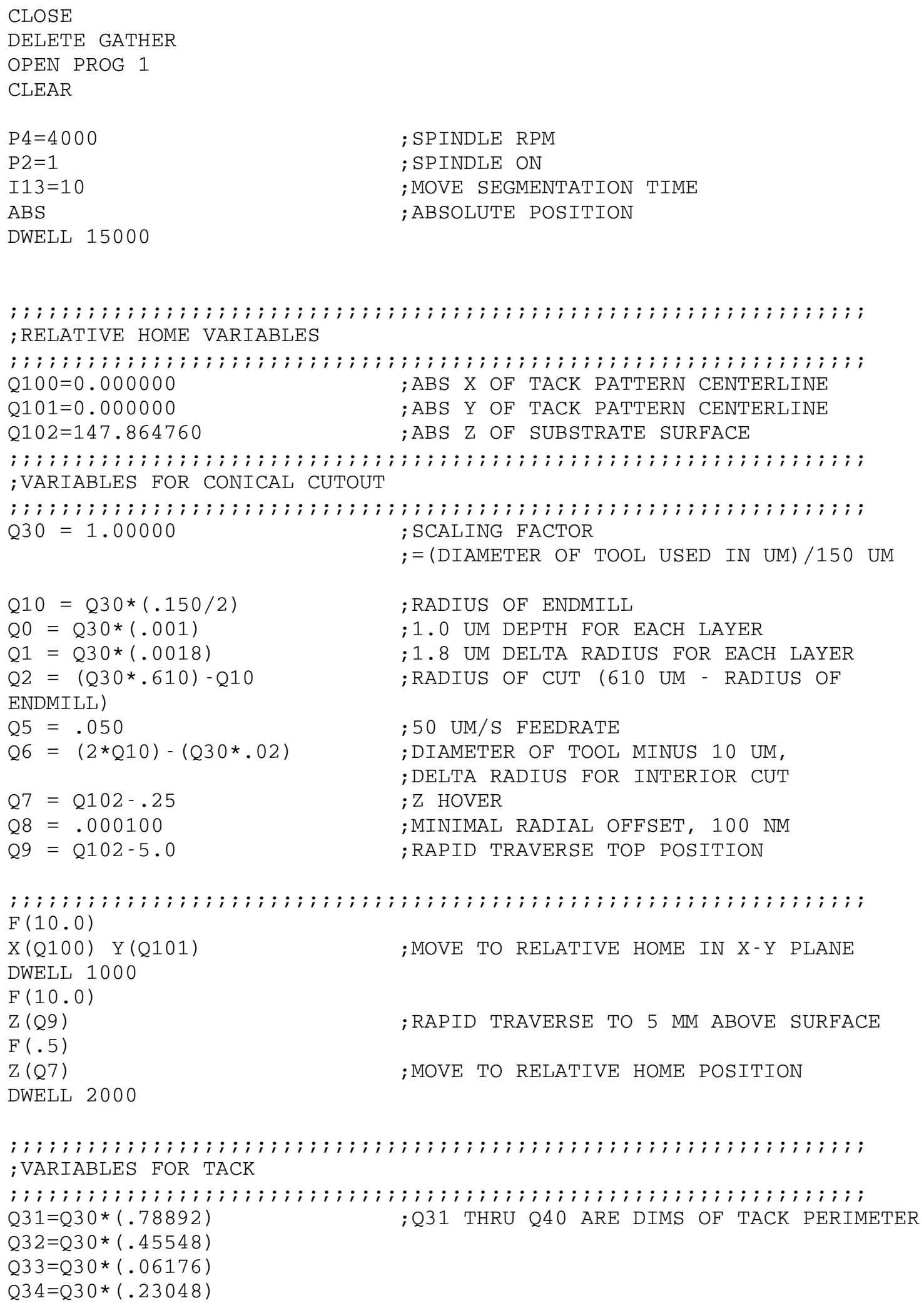




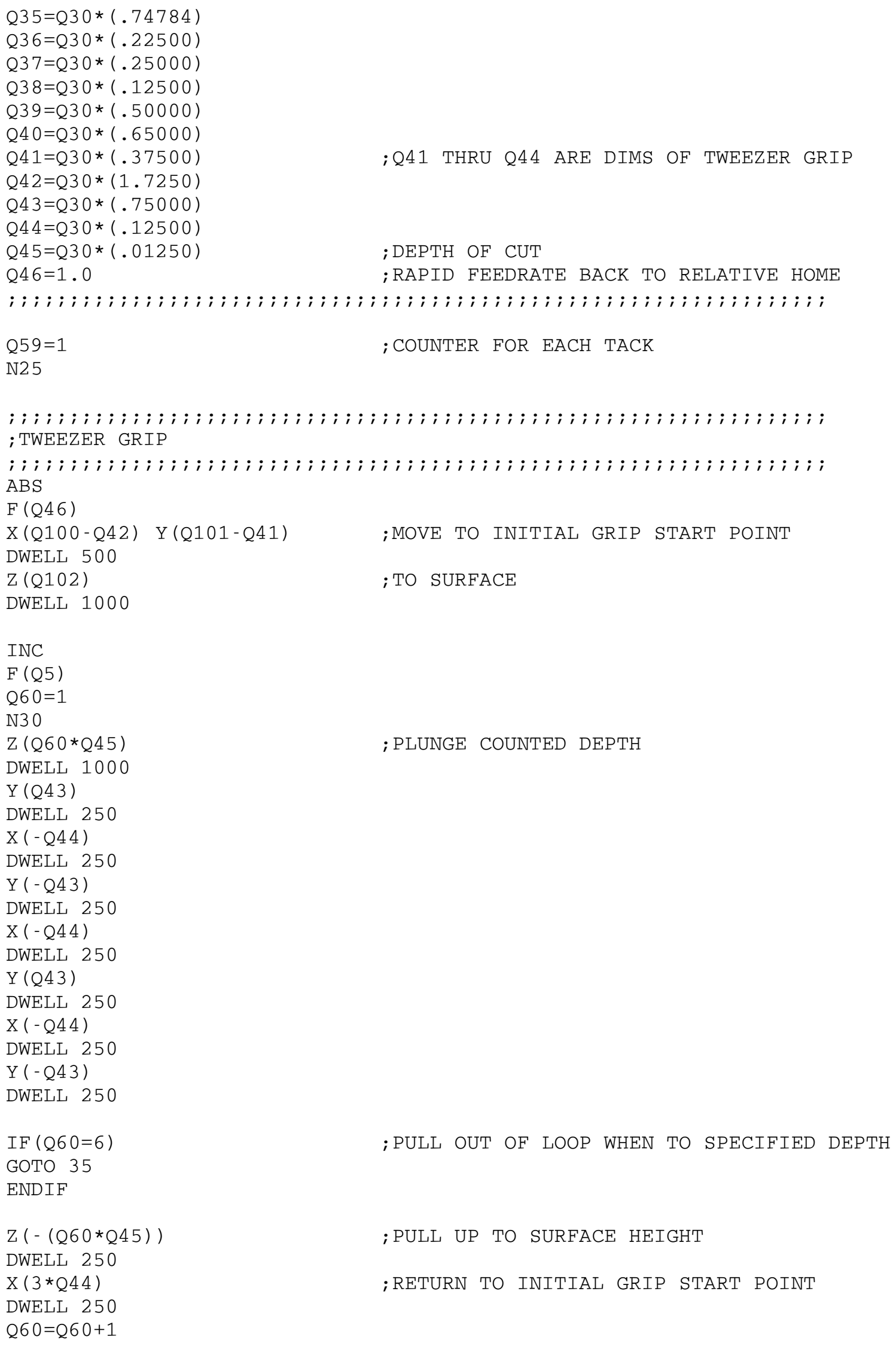




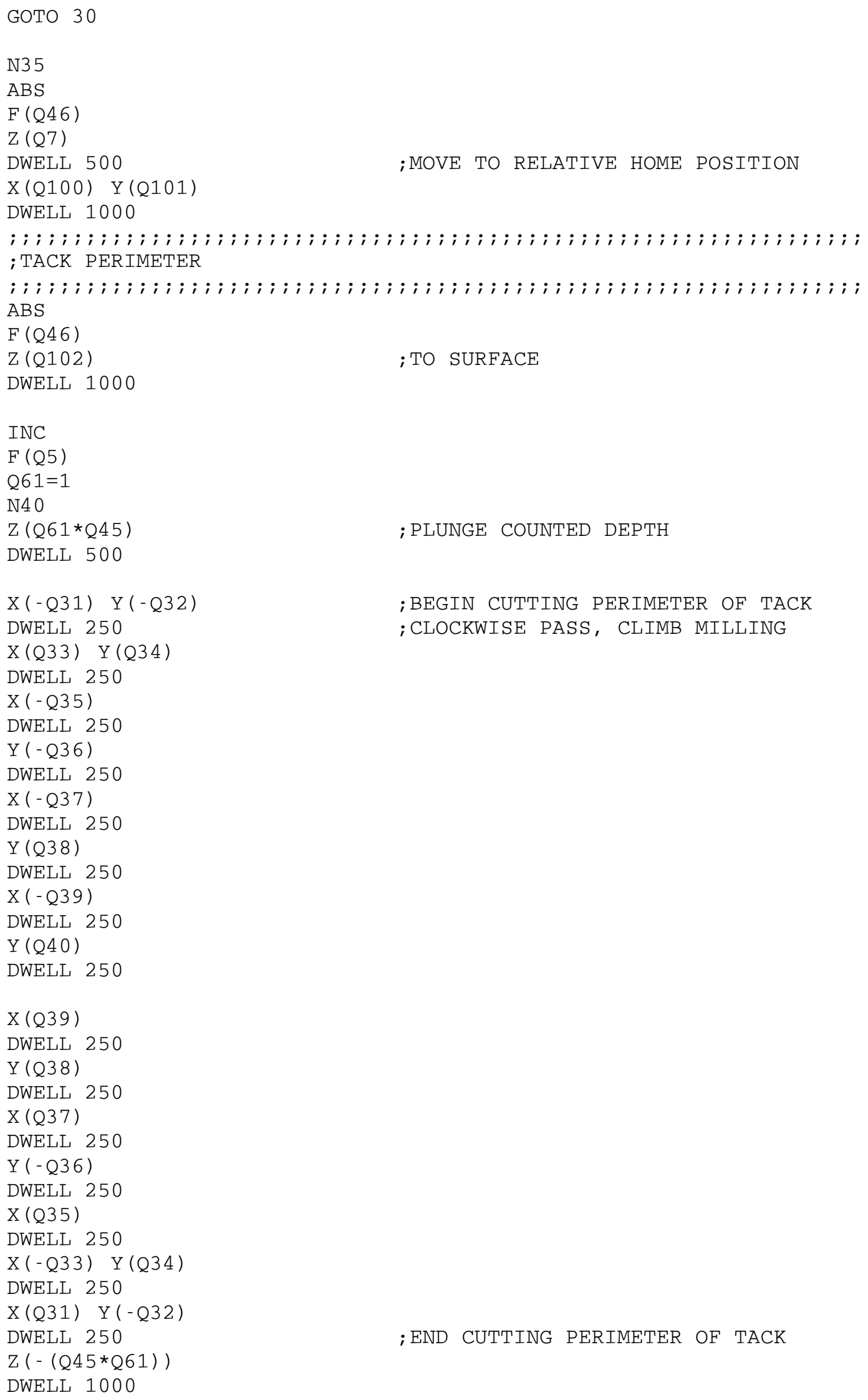

;BEGIN CUTTING PERIMETER OF TACK ;CLOCKWISE PASS, CLIMB MILLING

; END CUTTING PERIMETER OF TACK 


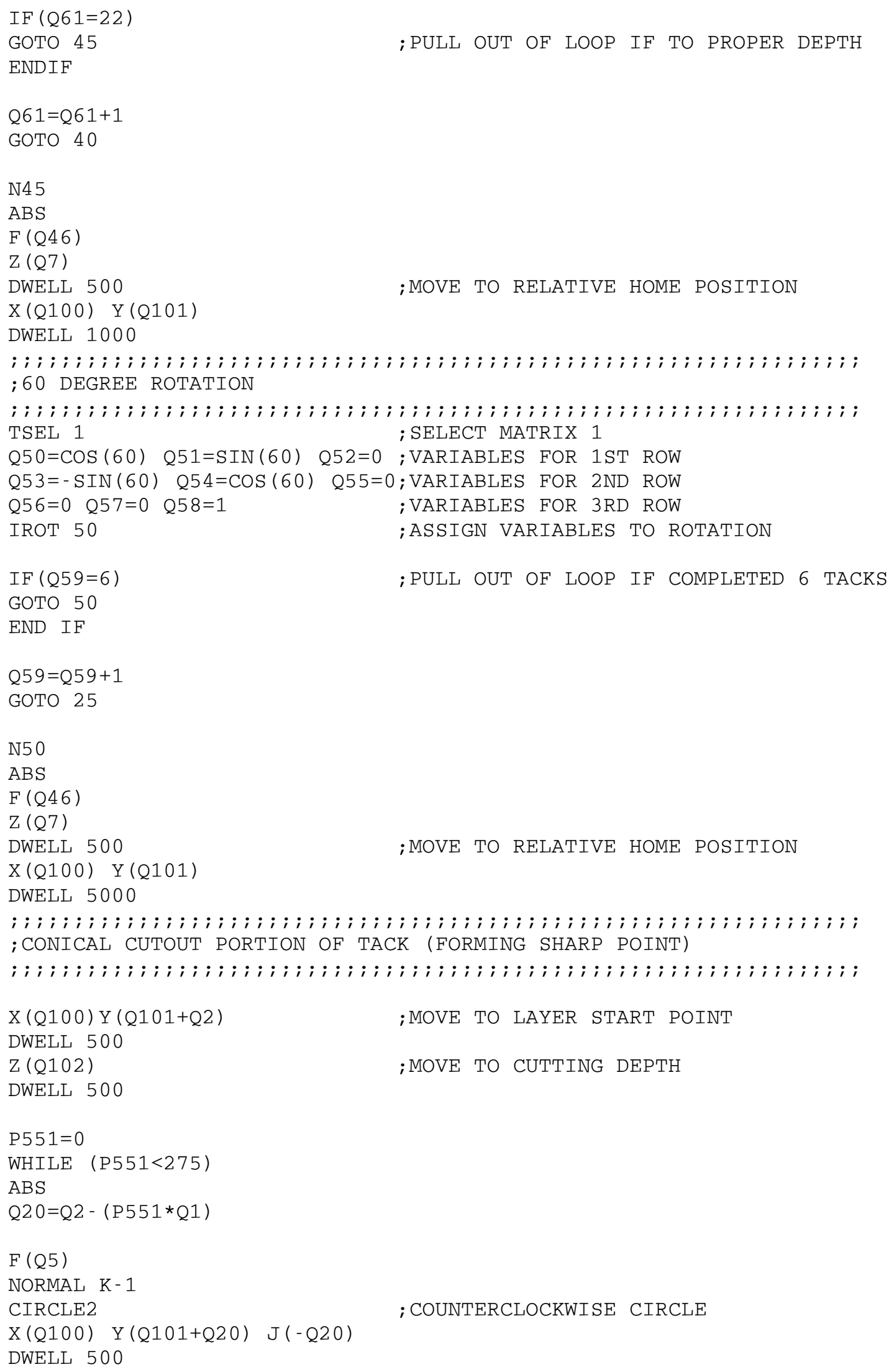


INC

$\mathrm{Y}(-\mathrm{Q} 1)$

DWELL 200

$\mathrm{Z}(\mathrm{Q} 0)$

DWELL 200

P551=P551+1

ENDWHILE

ABS

$F(.5)$

Z (Q7)

DWELL 1000

$X(Q 100) \quad Y(Q 101)$

DWELL 1000

$F(5.0)$

$\mathrm{Z}(-50)$

$\mathrm{P} 2=0$

; TURN OFF SPINDLE

CLOSE

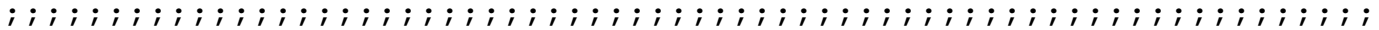


APPENDIX II

BUCKLING CRITERIA 


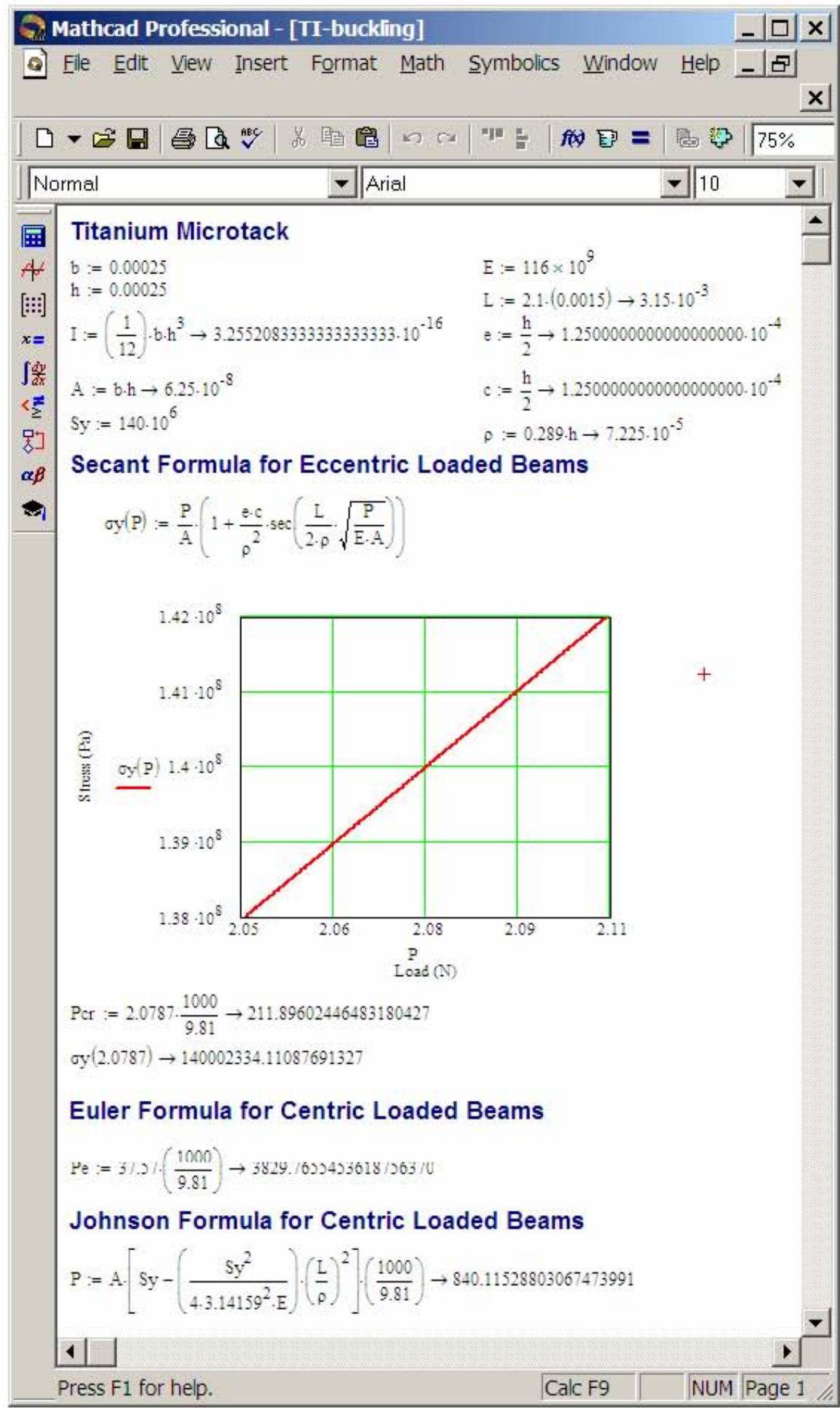

Figure 57. Buckling criteria for titanium microtack 


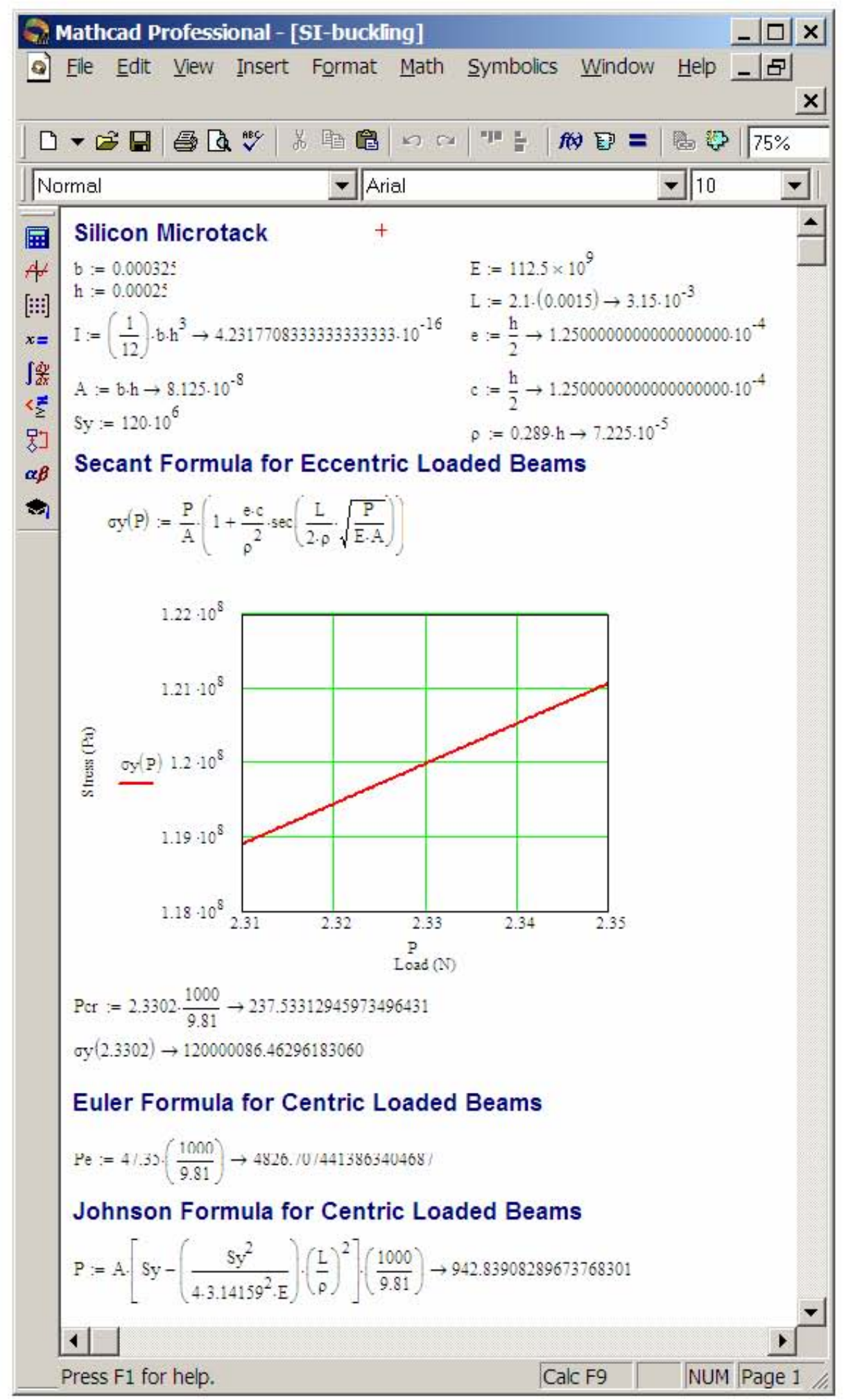

Figure 58. Buckling criteria for silicon pointed tip microtack 


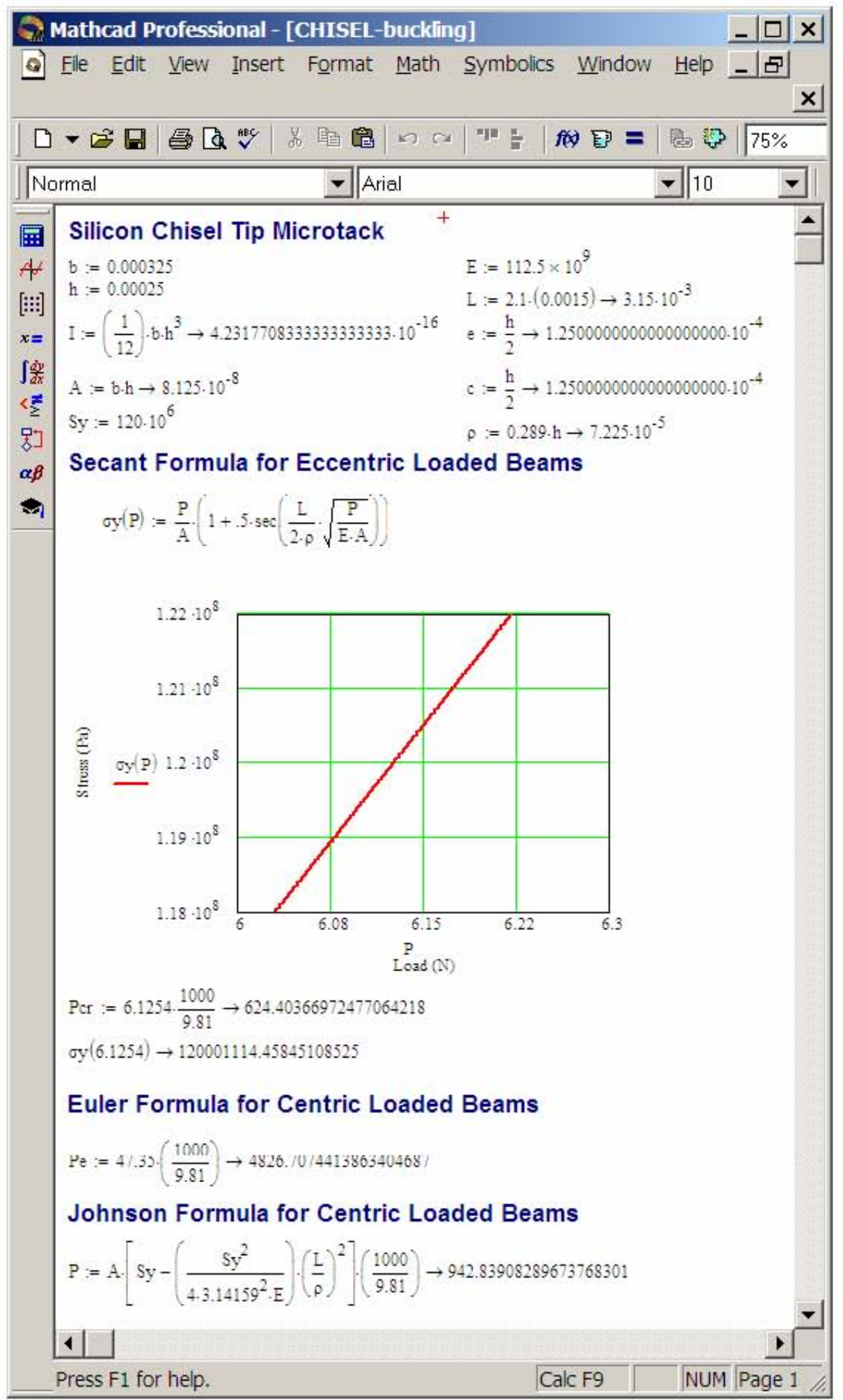

Figure 59. Buckling criteria for silicon chisel tip microtack 
RSA3 CALIBRATION 
1.) Open RSA III Orchestrator software.

2.) From Main Menu, click Utilities tab, got to Calibrate Instrument.

3.) Select transducer to be calibrated (Figure 54).

4.) Click XducerCAl command button.

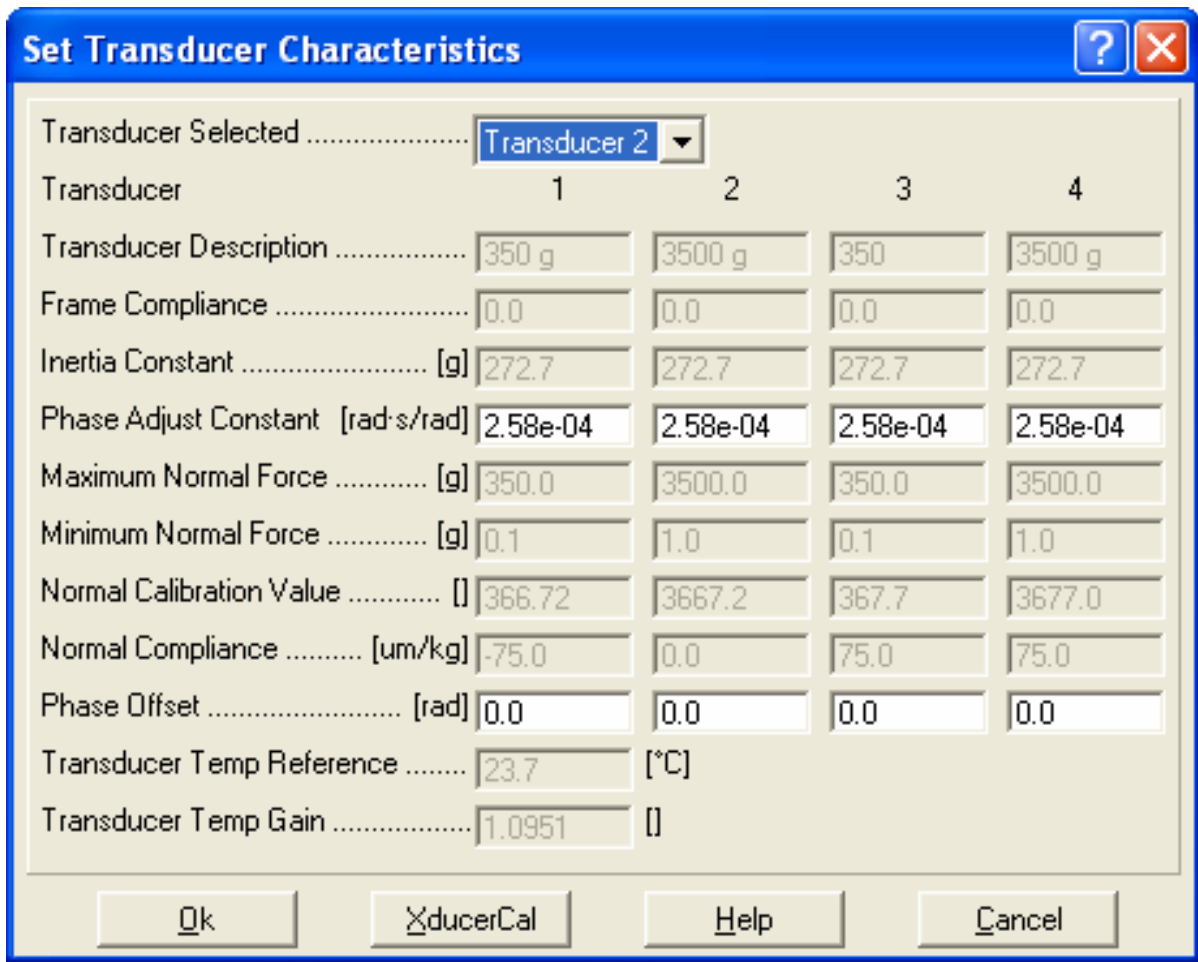

Figure 60. Set Transducer Characteristics window for transducer calibration on RSA3.

5.) Hang the calibration weight that corresponds to selected transducer:

- Transducer $1 \rightarrow 3667$ gram mass

- Transducer $2 \rightarrow 367$ gram mass.

6.) Press Force Cal command button to calibrate the selected transducer (Figure 55).

$$
\text { Transducer Calibration }
$$

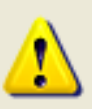

Hang calibration weight and press the Force Cal button to calibrate.

Current Force $\mathrm{Cal}=3667.2$

$$
\text { Force }[\mathrm{g}]=-1.0 \mathrm{e}+05 \quad \text { Temp. }\left[{ }^{\circ} \mathrm{C}\right]=27.191
$$

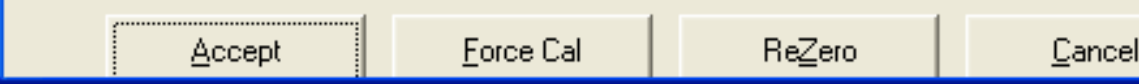

Figure 61. Transducer Calibration window

7.) Press Accept command button to accept calibration. 


\section{RUBBER SAMPLE TENSILE TEST DATA}


Table 8. Fiber reinforced synthetic rubber gasket material tensile tests to determine amount of tension applied to experiment samples

\begin{tabular}{|c|c|c|}
\hline TRIAL & $\begin{array}{c}\text { MAXIMUM TENSION } \\
\text { (grams) }\end{array}$ & $\begin{array}{c}\text { MAXIMUM DISPLACEMENT } \\
\text { (mm) }\end{array}$ \\
\hline $\mathbf{1}$ & 1791.95 & 8.002 \\
\hline $\mathbf{2}$ & 1778.19 & 8.002 \\
\hline $\mathbf{3}$ & 1758.07 & 8.002 \\
\hline $\mathbf{4}$ & 1743.63 & 8.002 \\
\hline $\mathbf{5}$ & 1732.31 & 8.002 \\
\hline $\mathbf{6}$ & 1724.91 & 8.002 \\
\hline $\mathbf{7}$ & 1718.25 & 8.002 \\
\hline $\mathbf{8}$ & 1712.19 & 8.002 \\
\hline $\mathbf{9}$ & 1707.51 & 8.002 \\
\hline $\mathbf{1 0}$ & 1702.94 & 8.002 \\
\hline & & $\mathbf{8 . 0 0 2}$ \\
\hline AVERAGE & $\mathbf{1 7 3 7 . 0 0}$ & 0 \\
\hline SD & $\mathbf{3 0 . 4 9}$ & \\
\hline & & \multicolumn{2}{|c|}{} \\
\hline
\end{tabular}

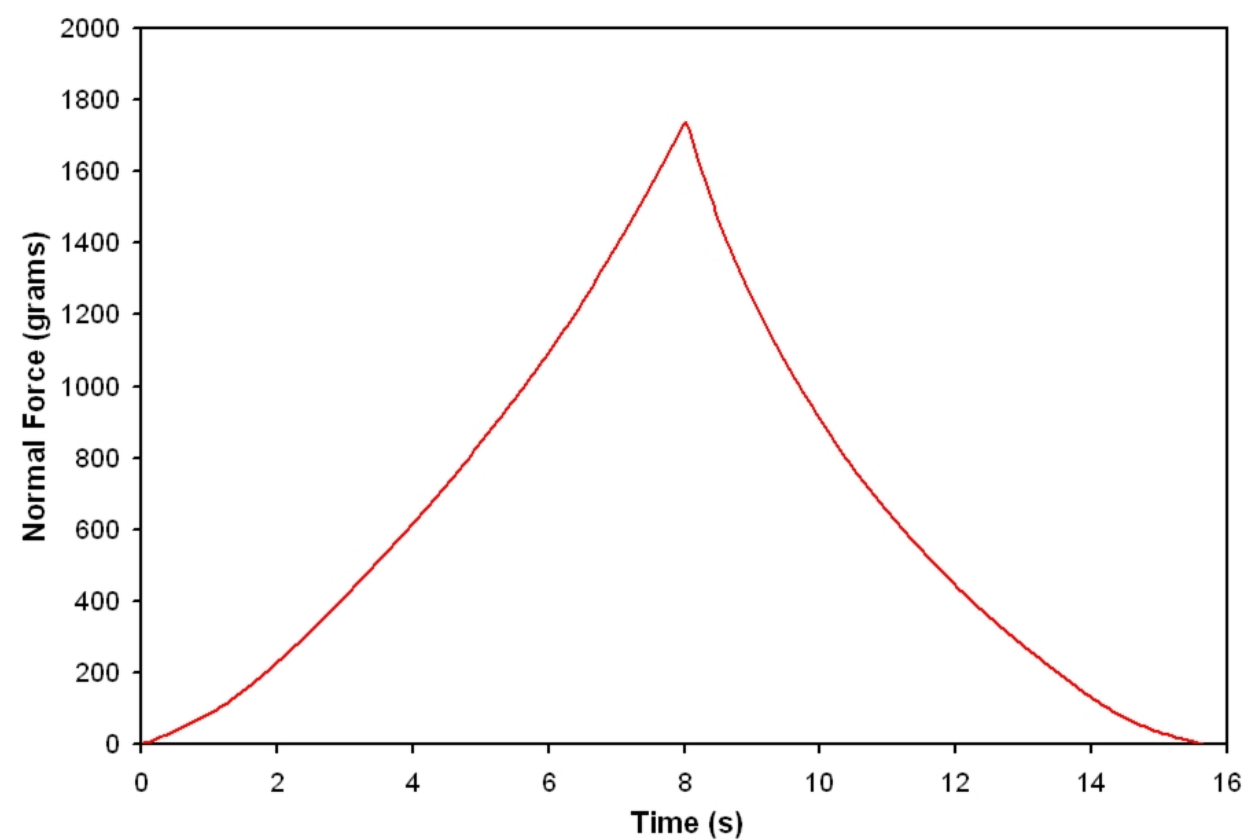

Figure 62. Normal force versus time of tensile tests to determine amount of tension applied to experiment samples 
TOTAL FORCE CALCULATION 


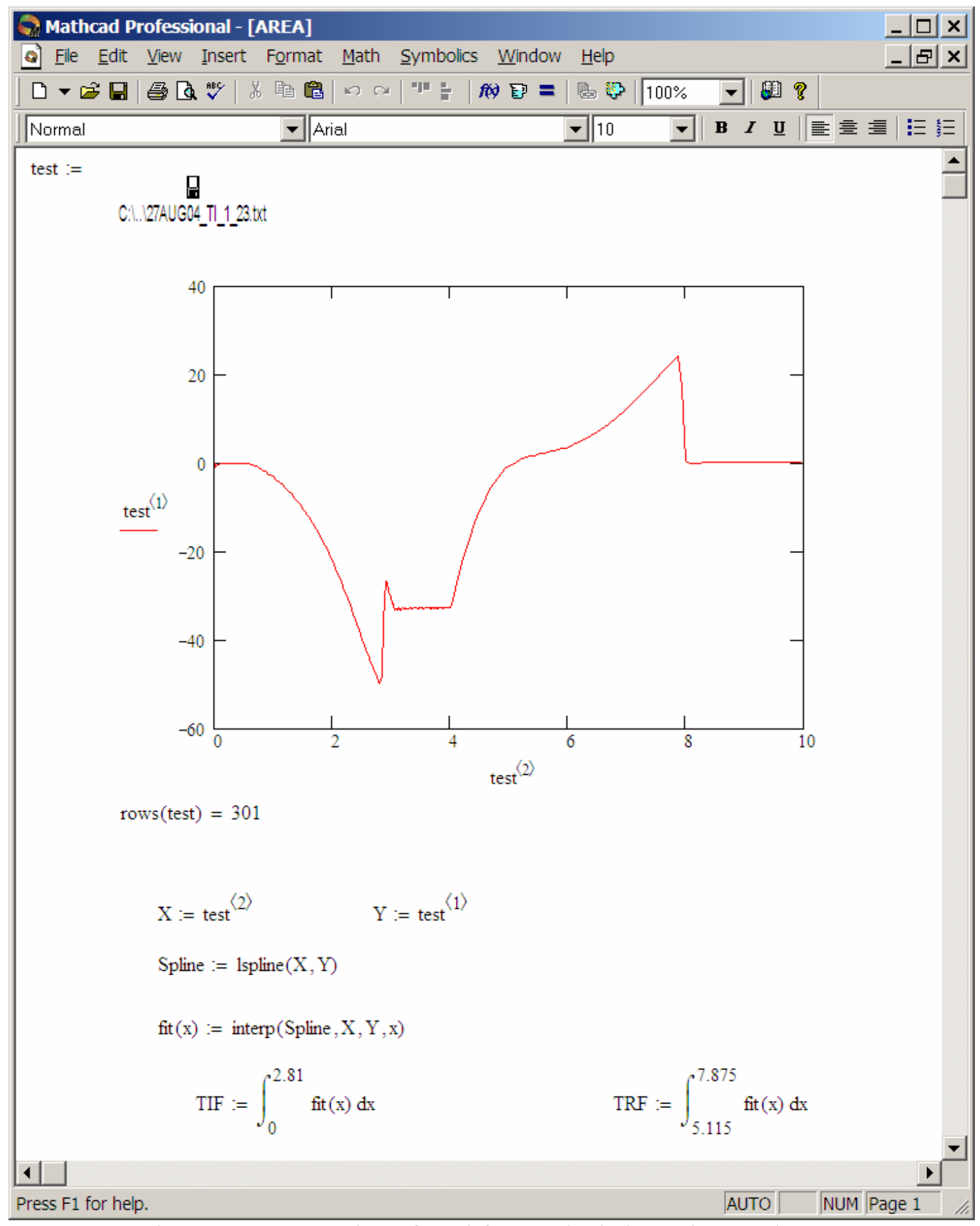

Figure 63. Screen shot of total force calculation using MathCAD 


\section{APPENDIX III}

SEM IMAGES 


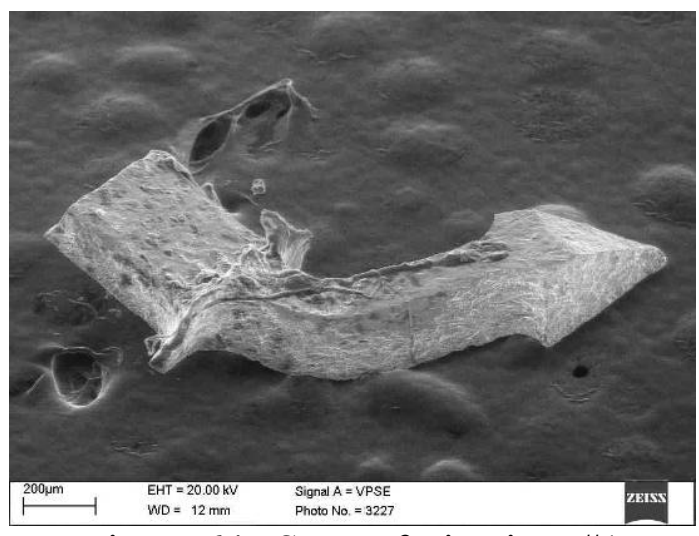

Figure 64. SEM of Titanium \#1

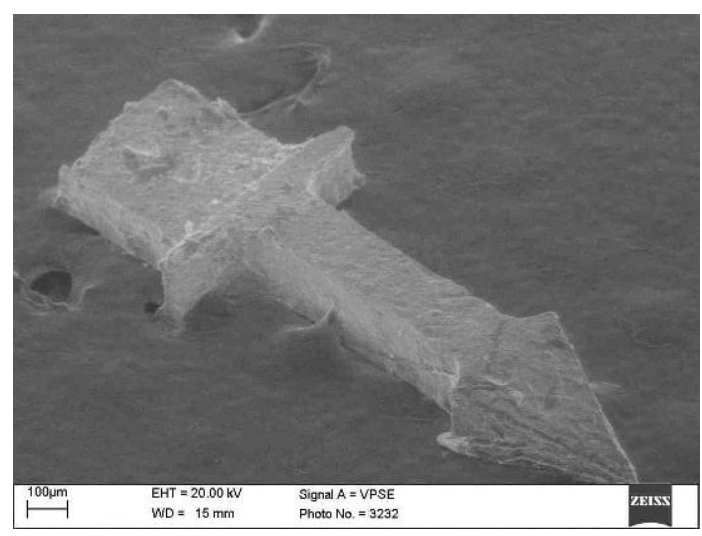

Figure 66. SEM of Titanium \#3

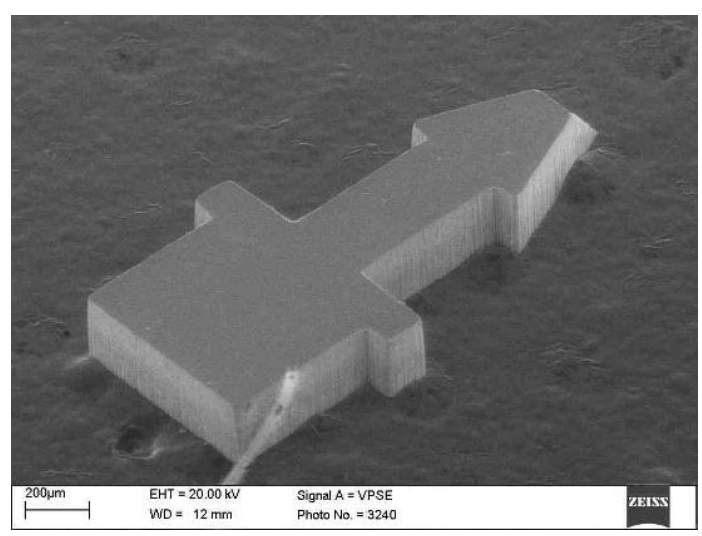

Figure 68. SEM of Silicon \#1

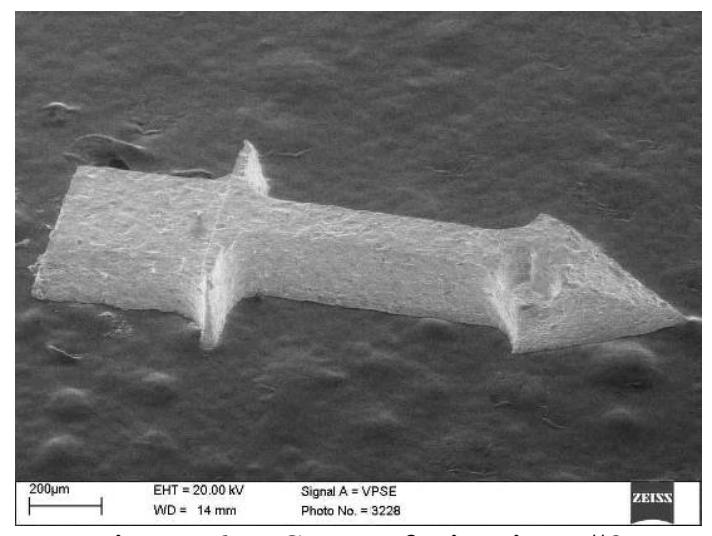

Figure 65. SEM of Titanium \#2

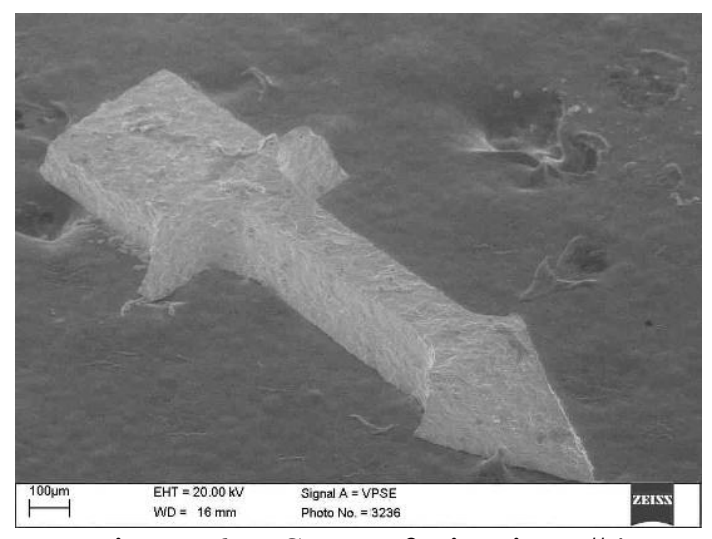

Figure 67. SEM of Titanium \#4

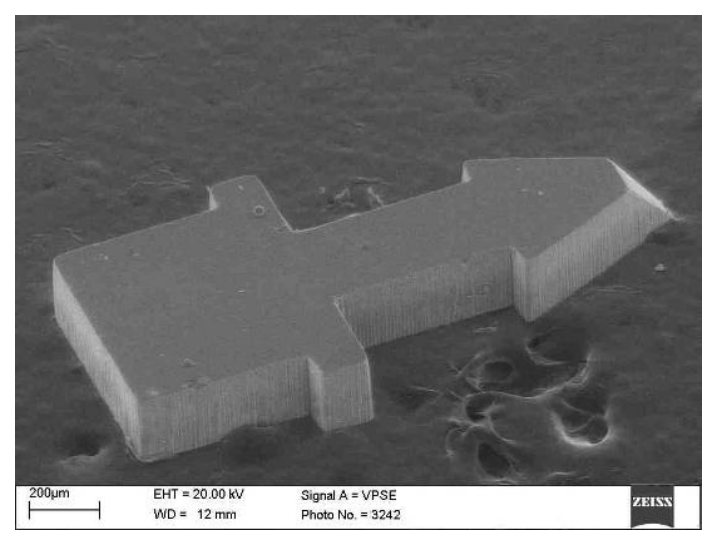

Figure 69. SEM of Silicon \#2 


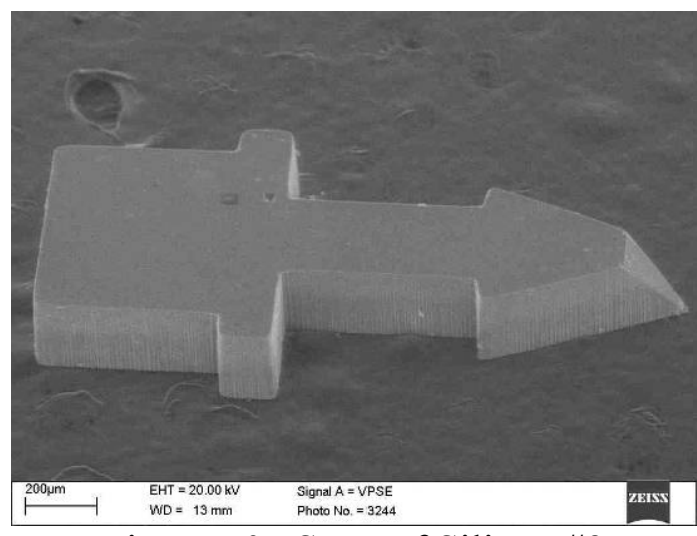

Figure 70. SEM of Silicon \#3

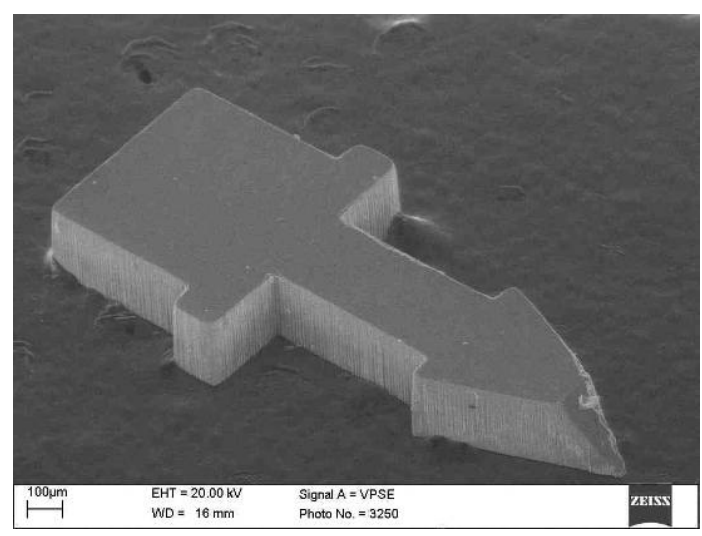

Figure 72. SEM of Silicon \#5

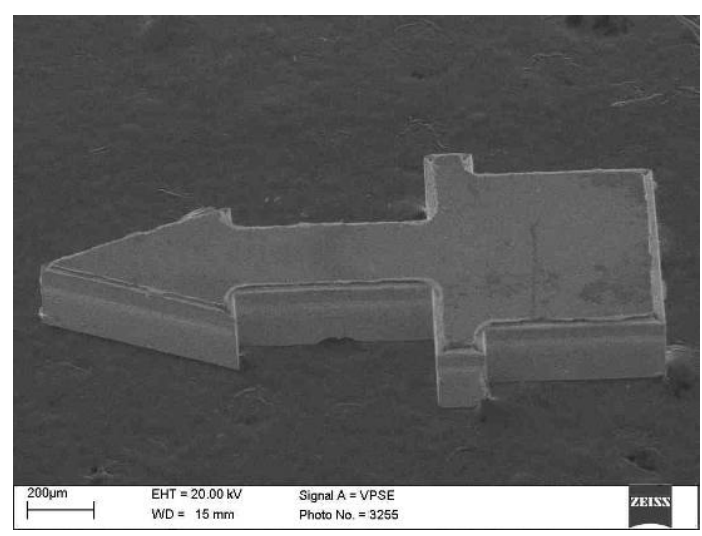

Figure 74. SEM of Silicon Chisel \#2

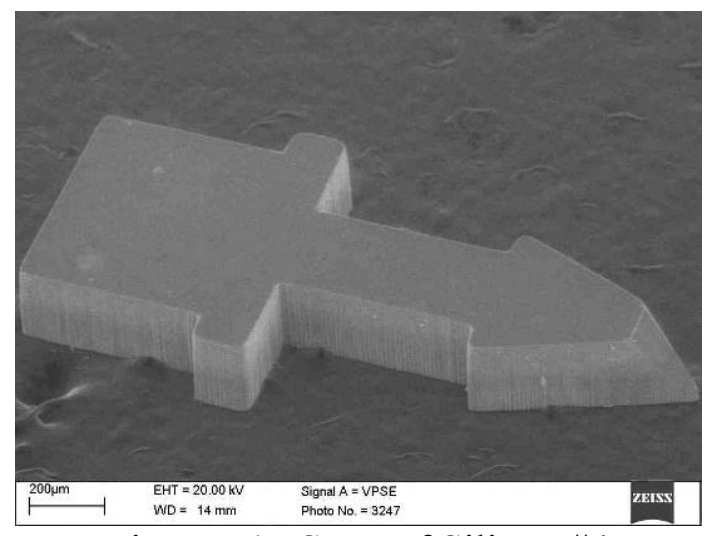

Figure 71. SEM of Silicon \#4

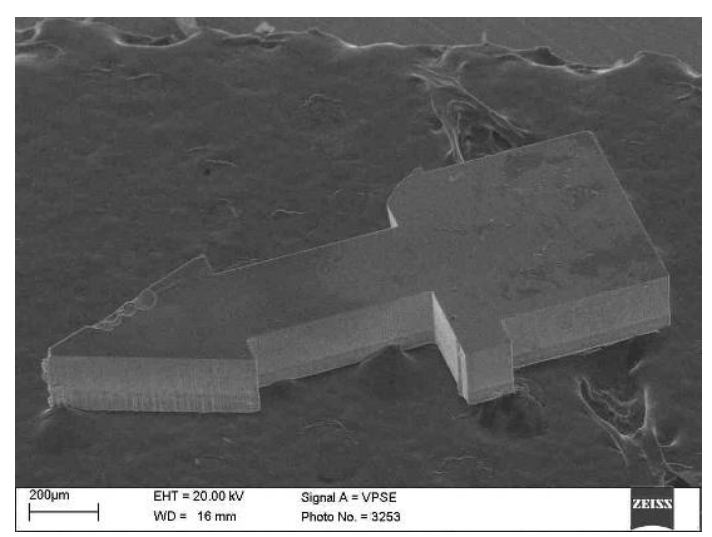

Figure 73. SEM of Silicon Chisel \#1

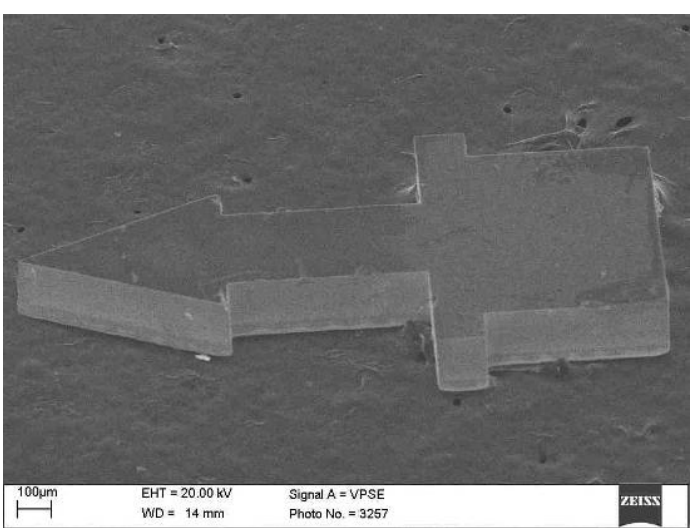

Figure 75. SEM of Silicon Chisel \#3 


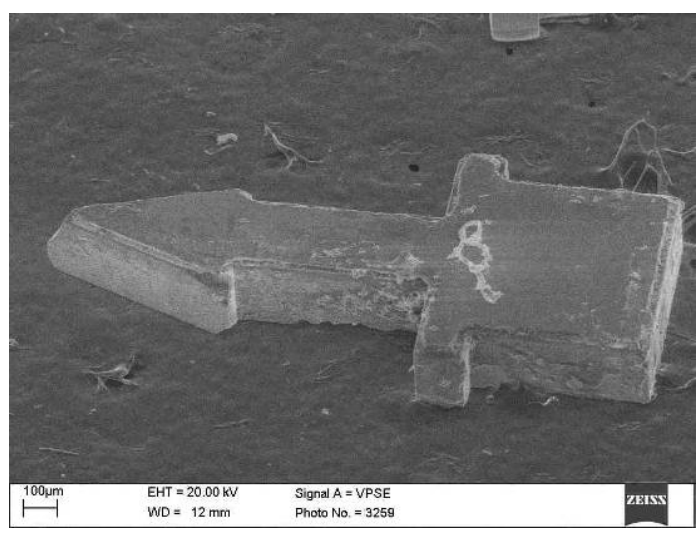

Figure 76. SEM of Silicon Chisel \#4

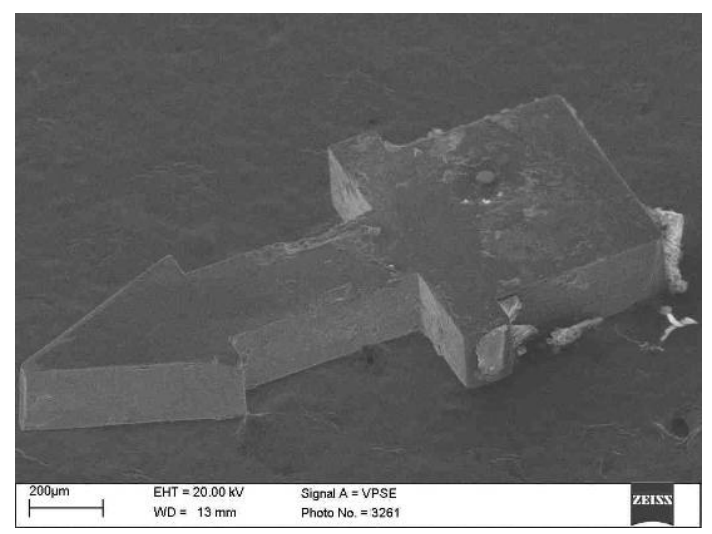

Figure 77. SEM of Silicon Chisel \#5

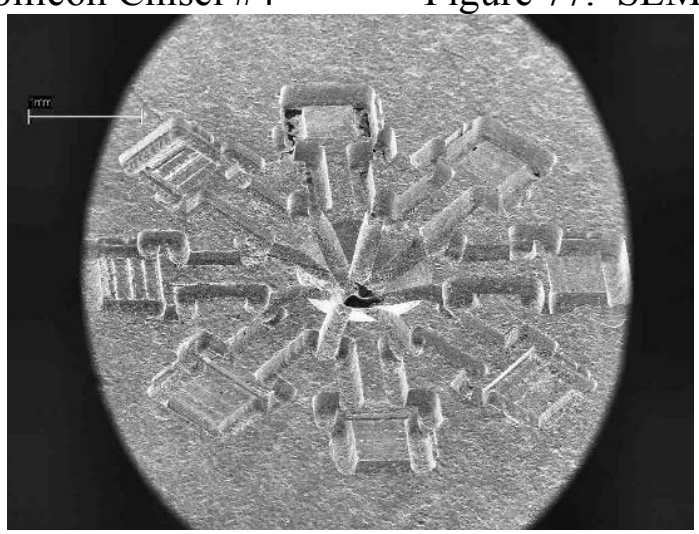

Figure 78. SEM image of array of Ti microtacks 
APPENDIX IV

FORCE DATA 


\section{AIV.1 Individual Microtack Data Tested in Fiber Reinforced Synthetic Rubber Gasket Material}

Table 9. Maximum insertion and removal forces for each test sample of each microtack design in fiber reinforced synthetic rubber gasket material

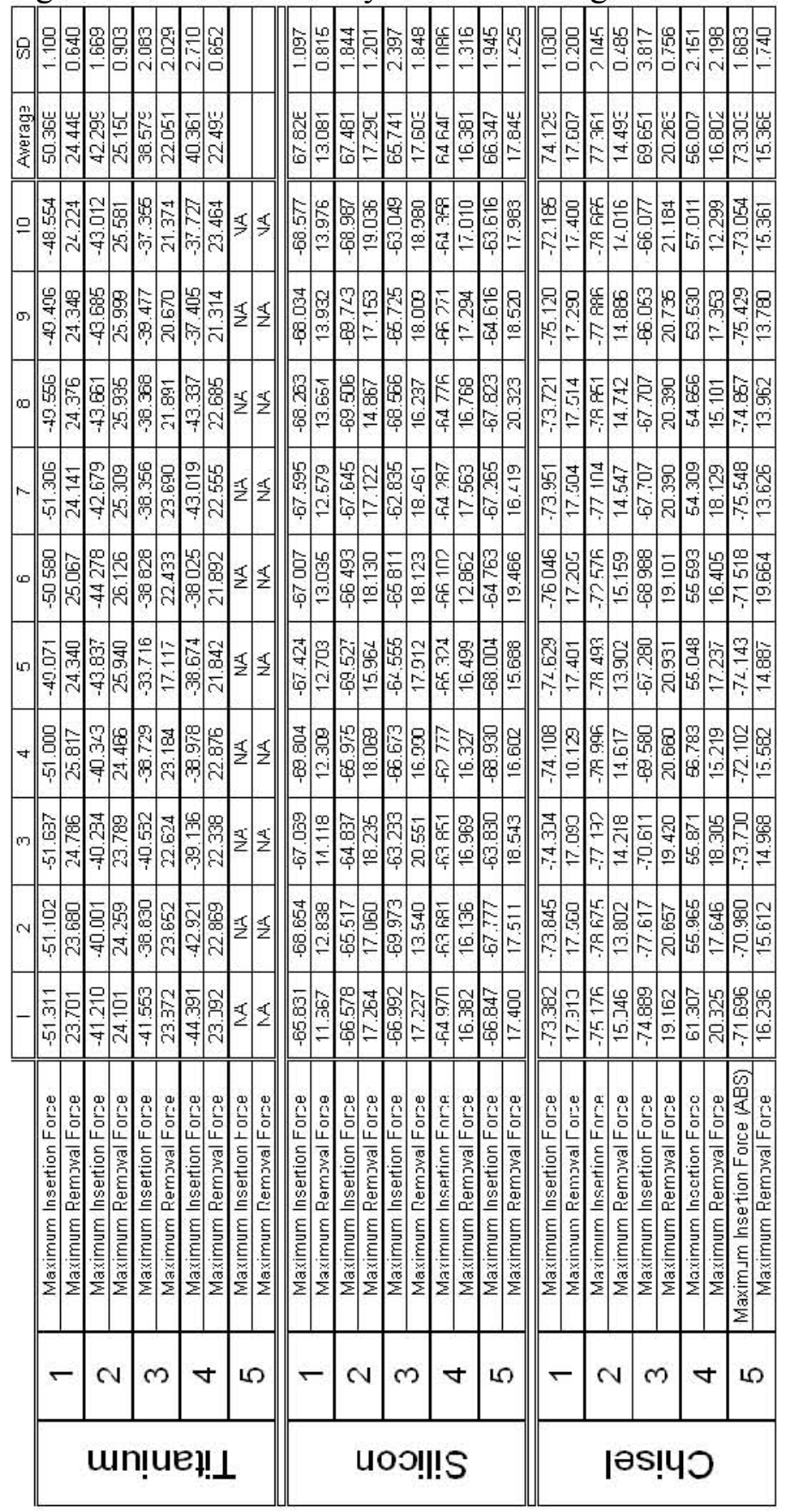


Table 10. Displacement at maximum insertion and removal forces for each test sample of each microtack design in fiber reinforced synthetic rubber gasket material

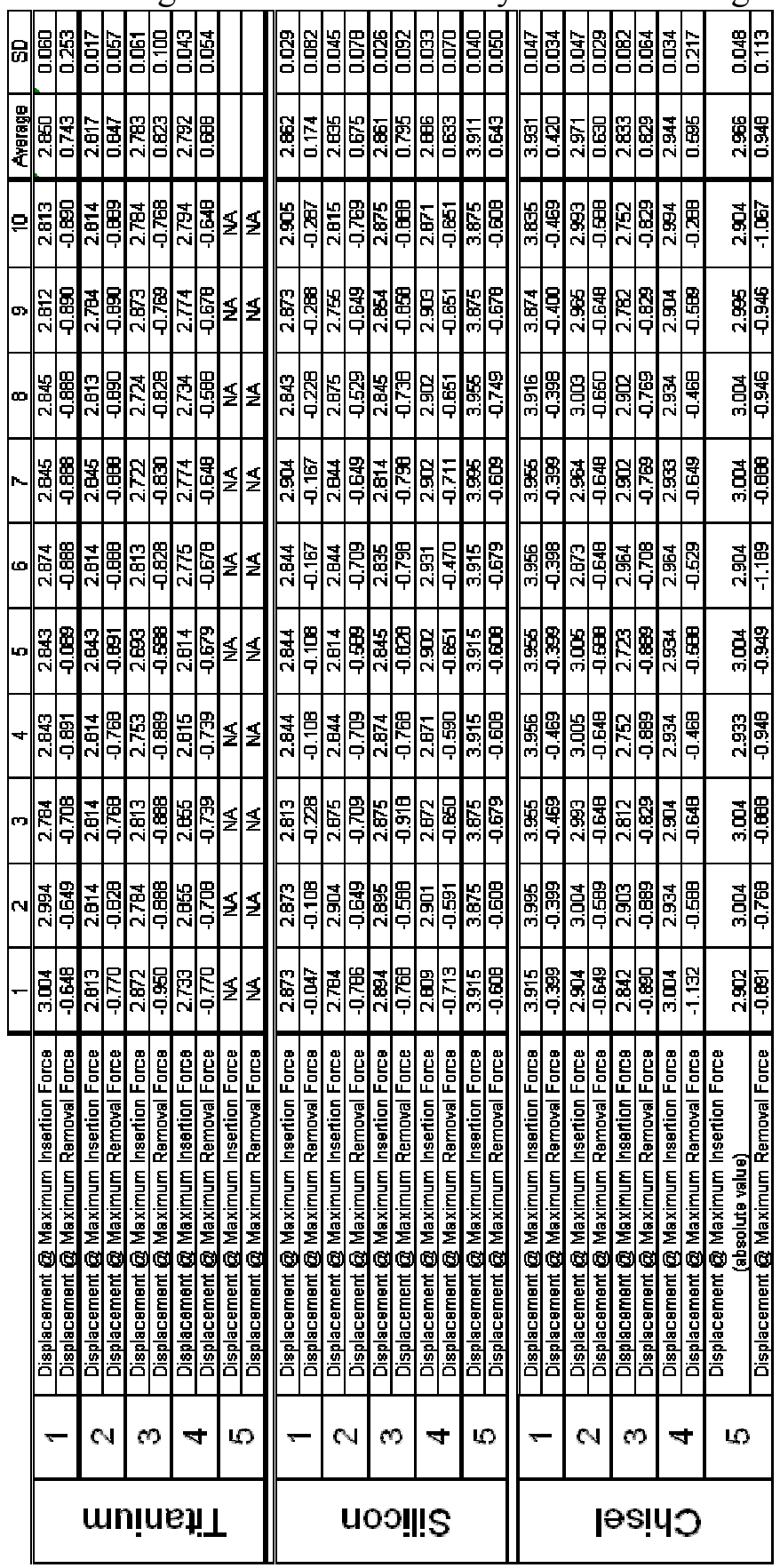


Table 11. Total insertion and removal forces for each test sample of each microtack design in fiber reinforced synthetic rubber gasket material

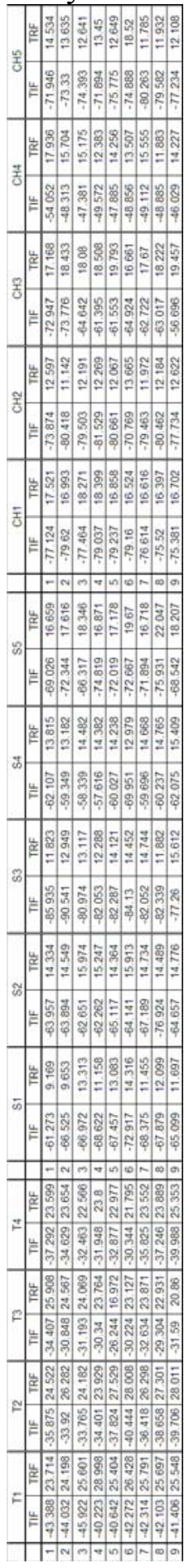




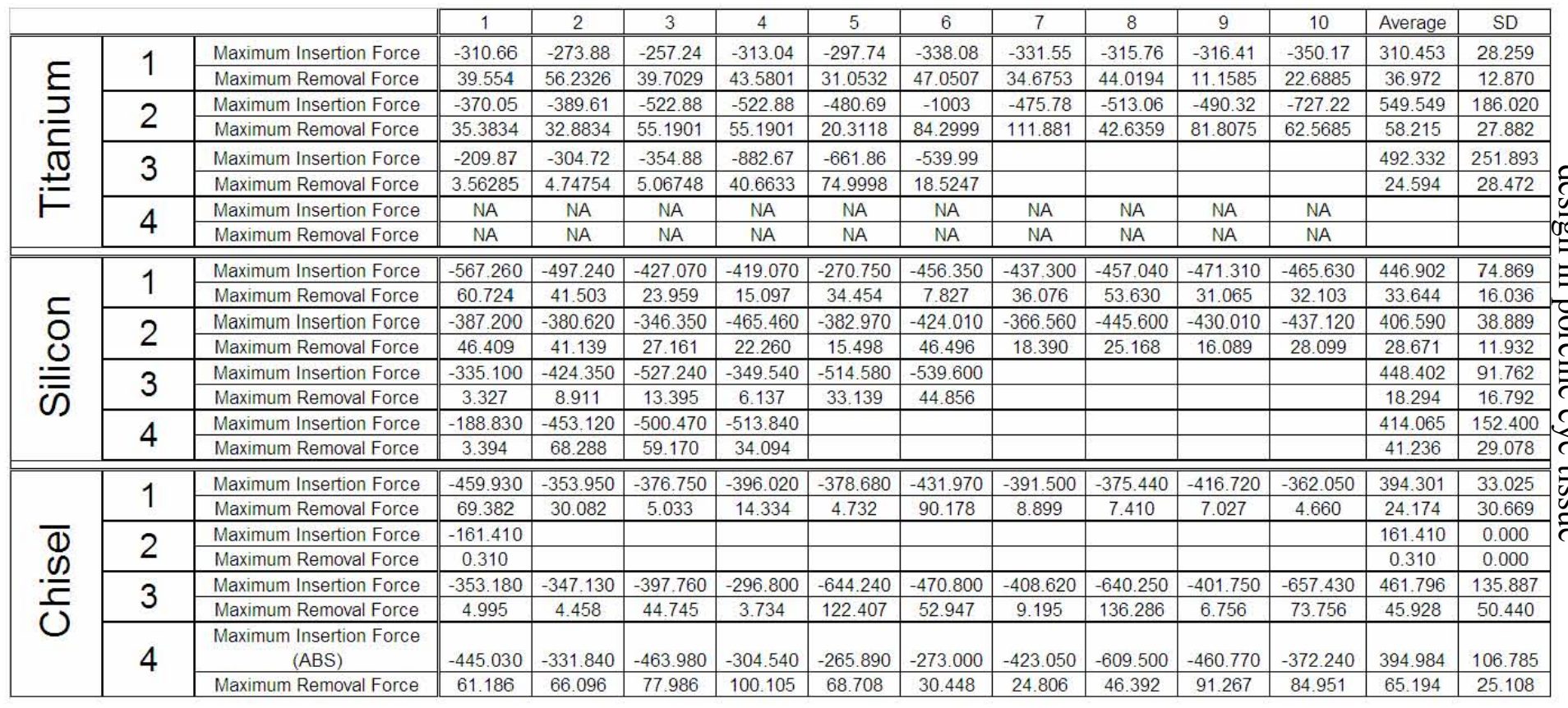

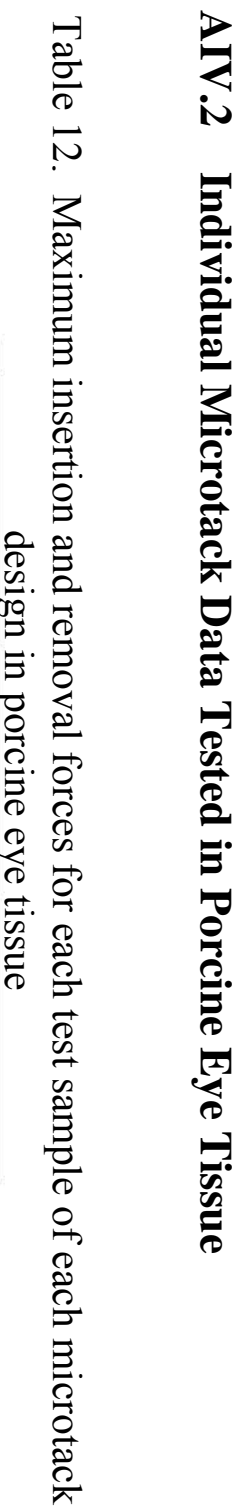


Table 13. Displacement at maximum insertion and removal forces for each test sample of each microtack design in porcine eye tissue

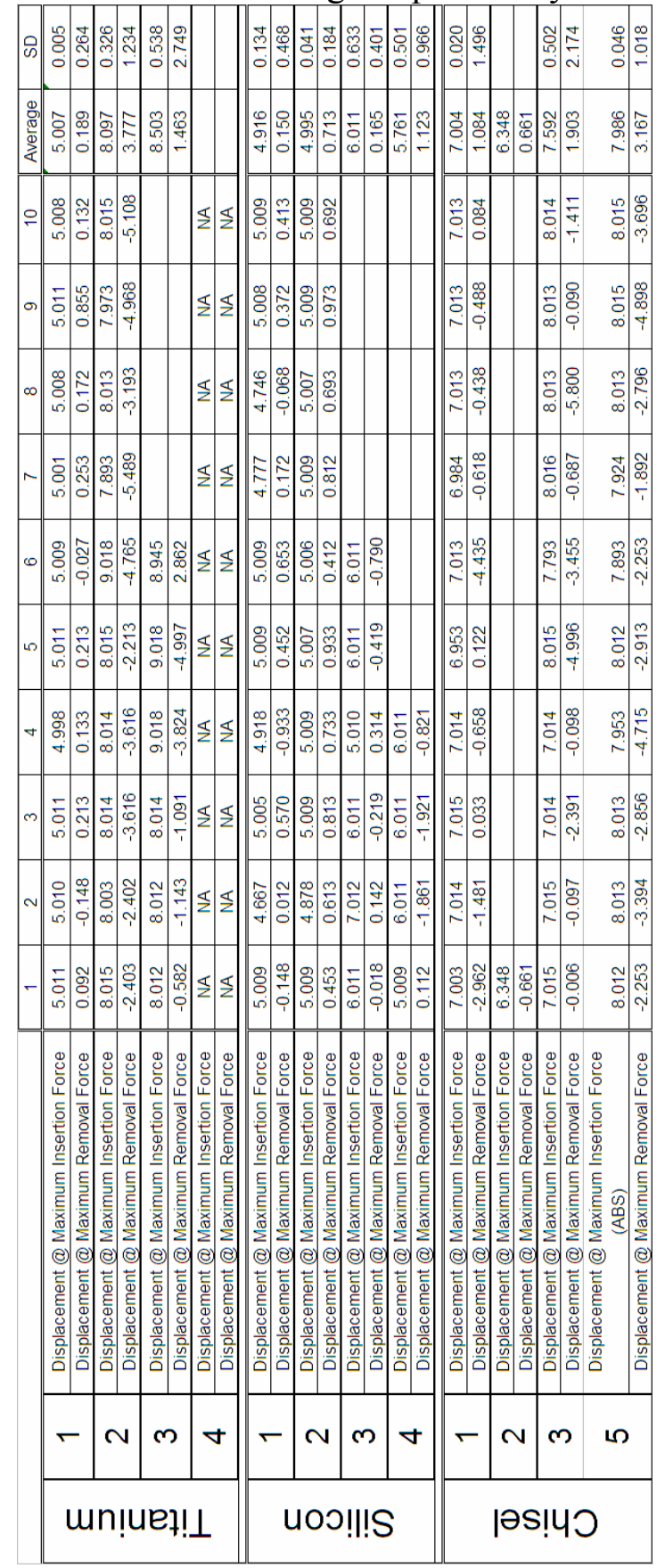


Table 14. Total insertion and removal forces for each test sample of each microtack design in porcine eye tissue

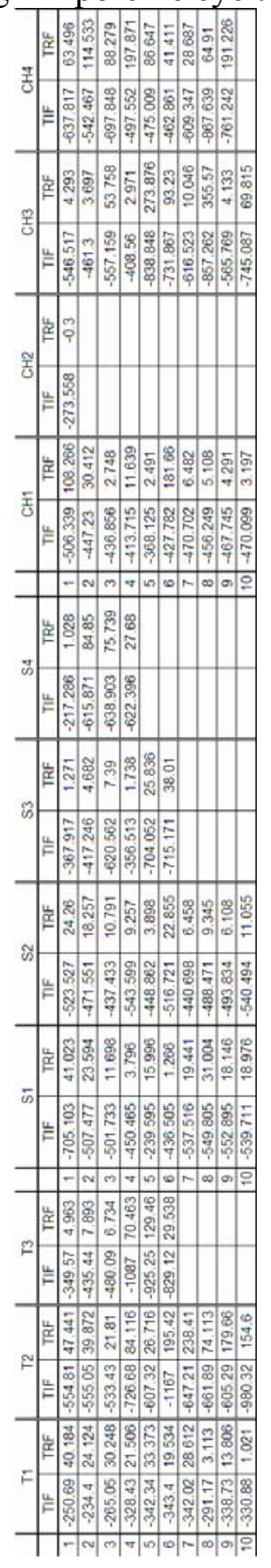




\section{VITA}

Scott Douglas Cambron was born on July 24, 1980 in Lebanon, Kentucky to Doug and Janice Cambron. He has four older sisters, Terri Miles, Renee Edelen, Ramona Smothers, and Regina Medley and one younger sister, Kimberly Cambron. He attended high school at Marion County High School from 1994 to 1998 when he graduated. He began his higher education at the University of Louisville in 1998. In the summer of 1999 he began his undergraduate research assistantship in the BioMEMS and Cardiovascular Mechanics Laboratory, under the guidance of Dr. Robert Keynton. He completed 2 co-ops with Samtec Inc. located in New Albany, IN, and the third within the BioMEMS and Cardiovascular Mechanics Laboratory directed by Dr. Robert Keynton at the University of Louisville, KY. In August of 2003, he received his Bachelor of Science in Mechanical Engineering. He received his Engineer-In-Training certificate in December 2003. He received his Master of Engineering degree in Mechanical Engineering with honors in May 2007.

Over his occupancy at Speed School of Engineering, he was involved in the University chapter of American Society of Mechanical Engineers (ASME), served in various positions, including Vice President during the 2003-2004 academic year. He was also involved in the University chapter of Society of Automotive Engineers (SAE), served as Vice President during the 2003-2004 academic year. He was a member of the 2003 and 2004 UofL SAE Mini Baja Team, where he assisted the team in the reinstatement of the University's participation in the SAE Mini Baja internationally sanctioned competitions. 
Future plans include pursuing the Doctor of Philosophy in Mechanical Engineering with a focus in Bioengineering while continuing his career as a Research Engineer in the newly developed Bioengineering Department at Speed School of Engineering. 